\title{
2002s-63
}

\section{Testing Normality: A GMM Approach}

\author{
Christian Bontemps and Nour Meddahi
}

Série Scientifique
Scientific Series

\section{Montréal}

Juillet 2002

(C) 2002 Christian Bontemps, Nour Meddahi. Tous droits réservés. All rights reserved. Reproduction partielle permise avec citation du document source, incluant la notice (C)

Short sections may be quoted without explicit permission, if full credit, including $@$ notice, is given to the source.
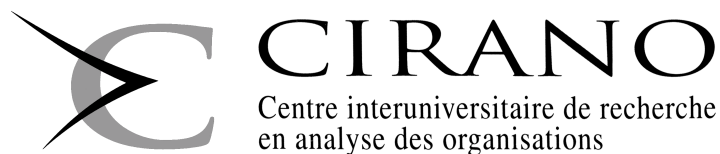

Centre interuniversitaire de recherche en analyse des organisations 


\section{CIRANO}

Le CIRANO est un organisme sans but lucratif constitué en vertu de la Loi des compagnies du Québec. Le financement de son infrastructure et de ses activités de recherche provient des cotisations de ses organisationsmembres, d'une subvention d'infrastructure du ministère de la Recherche, de la Science et de la Technologie, de même que des subventions et mandats obtenus par ses équipes de recherche.

CIRANO is a private non-profit organization incorporated under the Québec Companies Act. Its infrastructure and research activities are funded through fees paid by member organizations, an infrastructure grant from the Ministère de la Recherche, de la Science et de la Technologie, and grants and research mandates obtained by its research teams.

\section{Les organisations-partenaires / The Partner Organizations}

-École des Hautes Études Commerciales

-École Polytechnique de Montréal

-Université Concordia

- Université de Montréal

-Université du Québec à Montréal

-Université Laval

-Université McGill

-Ministère des Finances du Québec

-MRST

-Alcan inc.

-AXA Canada

-Banque du Canada

-Banque Laurentienne du Canada

-Banque Nationale du Canada

-Banque Royale du Canada

-Bell Canada

- Bombardier

-Bourse de Montréal

-Développement des ressources humaines Canada (DRHC)

-Fédération des caisses Desjardins du Québec

-Hydro-Québec

-Industrie Canada

-Pratt \& Whitney Canada Inc.

-Raymond Chabot Grant Thornton

-Ville de Montréal

Les cahiers de la série scientifique (CS) visent à rendre accessibles des résultats de recherche effectuée au CIRANO afin de susciter échanges et commentaires. Ces cahiers sont écrits dans le style des publications scientifiques. Les idées et les opinions émises sont sous l'unique responsabilité des auteurs et ne représentent pas nécessairement les positions du CIRANO ou de ses partenaires.

This paper presents research carried out at CIRANO and aims at encouraging discussion and comment.

The observations and viewpoints expressed are the sole responsibility of the authors. They do not necessarily represent positions of CIRANO or its partners. 


\title{
Testing Normality: A GMM Approach*
}

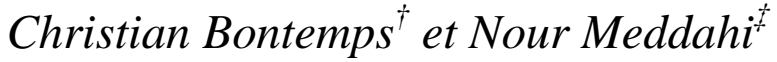

\begin{abstract}
Résumé / Abstract
Dans cet article, nous testons des hypothèses de normalité marginale. Plus précisément, nous proposons des tests fondés sur des conditions de moments connues sous le nom d'équations de Stein. Ces conditions coïncident avec la première classe de conditions de moments obtenues par Hansen et Scheinkman (1995) quand la variable d'intérêt est une diffusion. L'équation de Stein implique, par exemple, que l'espérance de chaque polynôme de Hermite est nulle. L'approche GMM est utile pour deux raisons. Elle nous permet de tenir compte du problème d'incertitude des paramètres préalablement estimés. En particulier, nous caractérisons les conditions de moments qui sont robustes à ce problème et montrons que c'est le cas des polynômes de Hermite. C'est la principale contribution de l'article. Le second avantage de l'approche GMM est que nos tests sont aussi valides pour des séries temporelles. Dans ce cas, nous adoptons une approche HAC (Heteroskedastic-Autocorrelation-Consistent) pour estimer la matrice de poids qui intervient dans la statistique de test quand la forme sérielle des données n'est pas spécifiée. Nous comparons nos tests de manière théorique avec les tests de Jarque et Bera (1981) et les tests dits OPG de Davidson et MacKinnon (1993). Les propriétés de petits échantillons de nos tests sont étudiées par simulation. Finalement, nous appliquons nos tests à trois exemples de modèles de volatilité GARCH et volatilité réalisée.
\end{abstract}

In this paper, we consider testing marginal normal distributional assumptions. More precisely, we propose tests based on moment conditions implied by normality. These moment conditions are known as the Stein (1972) equations. They coincide with the first class of moment conditions derived by Hansen and Scheinkman (1995) when the random variable of interest is a scalar diffusion. Among other examples, Stein equation implies that the mean of Hermite polynomials is zero. The GMM approach we adopted is well suited for two reasons. It allows us to study in detail the parameter uncertainty problem, i.e., when the tests depend on unknown parameters that have to be estimated. In particular, we characterize the moment conditions that are robust against parameter uncertainty and show that Hermite polynomials are special examples. This is the main contribution of the paper. The second reason for using GMM is that our tests are also valid for time series. In this case, we adopt a Heteroskedastic-Autocorrelation-Consistent approach to estimate the weighting matrix when the dependence of the data is unspecified. We

\footnotetext{
* The authors would like to thank Manuel Arellano, Bryan Campbell, Marine Carrasco, Xiahong Chen, Russell Davidson, Jean-Marie Dufour, Jean-Pierre Florens, René Garcia, Ramazan Gençay, Silvia Gonçalves, Lynda Khalaf, James MacKinnon, Benoit Perron and Enrique Sentana for helpful comments on an earlier draft and participants at CEMFI, Université de Montréal, Queen's University and University of Pittsburgh seminars, the Canadian Economic Association Meeting, Montréal, June, 2001, and the European Econometric Society Meeting, Lausanne, August, 2001. The authors are responsible for any remaining errors. The second author acknowledges financial support from FCAR, IFM2, and MITACS.

$\dagger$ LEEA-CENA, 7 avenue Edouard Belin, 31055 Toulouse Cedex, FRANCE. E-mail: bontemps @ cena.fr; † Département de sciences économiques, CRDE, CIRANO, Université de Montréal, and CEPR. Address: C.P. 6128, succursale Centre-ville, Montréal (Québec), H3C 3J7, Canada. E-mail: nour.meddahi@ umontreal.ca.
} 
also make a theoretical comparison of our tests with Jarque and Bera (1980) and OPG regression tests of Davidson and MacKinnon (1993). Finite sample properties of our tests are derived through a comprehensive Monte Carlo study. Finally, three applications to GARCH and realized volatility models are presented.

Mots clés : Normalité, équation de Stein-Hansen-Scheinkman, GMM, polynômes de Hermite, incertitude des paramètres, HAC, régression OPG

Keywords: Normality, Stein-Hansen-Scheinkman equation, GMM, Hermite polynomials, parameter uncertainty, HAC, OPG regression

Codes JEL : C12, C15, C22 


\section{Introduction}

In many econometric models, distributional assumptions play an important role in the estimation, inference and forecasting procedures. Robust estimation methods against distributional assumption are available, such as the Quasi-Maximum-Likelihood (White, 1982; QML) and Generalized Method of Moments (Hansen, 1982; GMM). However, knowing the true distribution of the considered random variable may be useful for improving inference. Such is the case in stochastic volatility models where several studies have shown that simulation and Bayesian methods outperform the QML and GMM methods (Jacquier, Polson and Rossi, 1994; Kim, Shephard and Chib, 1998; Andersen, Chung and Sorensen, 1999; Gallant and Tauchen, 1999). Moreover, knowing the distribution is also crucial when one forecasts non linear variables like volatility in the EGARCH model of Nelson (1991), or the high frequency realized volatility model of Andersen, Bollerslev, Diebold and Labys (2002, ABDL). This is also important when one evaluates density forecasts as in Diebold, Gunther and Tay (1998). In risk management literature (see Christoffersen, 1998, and Berkowitz, 2001), the most popular measure of risk, that is the Value at Risk (VaR), is based on quantiles and, hence, on distributional assumptions. In continuous time modeling, Chen, Hansen and Scheinkman (2000) argue that an interesting approach is to first specify the unconditional distribution of the process, and then specify the diffusion term. Therefore, developing tests procedure for distributional assumption diagnostics in both cross-sectional and time-series settings is of particular interest.

The main purpose of our paper is to provide a new approach for testing normality. We consider normality given that its importance in the econometric literature. Moreover, econometricians are more familiar with testing normality. Finally, any continuous distribution may be transformed on a normal one.

There is a very important literature on testing normality. This includes tests based on the cumulative distribution (Kolmogorov, 1933; Smirnov, 1939), the characteristic function (Koutrouvelis, 1980; Koutrouvelis and Kellermeier, 1981; Epps and Pulley, 1983), the moment generating function (Epps, Singleton and Pulley, 1982), the third and fourth moment (Mardia, 1970; Bowman and Shenton, 1975; Jarque and Bera, 1980; Bera and Jarque, 1981), the Hermite polynomials (Kiefer and Salmon, 1983; van der Klaauw and Koning, 2001). ${ }^{1}$

Our approach is based on testing moment conditions. The conditions we consider are based on Stein (1972), where it is showed that the marginal distribution of a random variable is normal with zero mean and unit variance if and only if a particular set of moment conditions hold. Each moment condition is known as the Stein equation (see for instance Schoutens, 2000). We show that special examples of this equation correspond to the zero mean of any Hermite polynomials. Interestingly, the Stein equation coincides with the first class of moment

\footnotetext{
${ }^{1}$ Multivariate tests are also based on the third and fourth moments (Mardia, 1970; Bera and John, 1983; Richardson and Smith, 1993; Kilian and Demiroglu, 2000; Fiorentini, Sentana and Calzolari, 2002).
} 
conditions given by Hansen and Scheinkman (1995) for continuous time processes when one considers a normal process, that is the Ornstein-Uhlenbeck process. ${ }^{2}$

We use the GMM approach for testing the Stein equation. The GMM approach is very appealing for two reasons. It is well suited for correcting the test statistic distribution when one uses estimated parameters. Moreover, it is easy in the GMM setting to take into account potential dependence in the data when one tests marginal moment conditions.

In general, the normality assumption is made for unobservable variables. Hence, one has to estimate the model parameters and then test normality on the fitted variables such as the residuals. As a consequence, one has to take into account the parameter uncertainty, since it is well known that in general the distribution of the test statistic is not the same when one uses the true parameter and an estimator. This problem leads for instance Lilliefors (1967) to tabulate the Kolmogorov-Smirnov test statistic when one estimates the mean and the variance of the distribution. In the linear homoskedastic model, White and MacDonald (1980) showed that various tests are robust against parameter uncertainty, in particular tests based on moments that used standardized residuals. Finally, note that Dufour, Farhat, Gardiol and Khalaf (1998) developed Monte Carlo tests to take into account parameter uncertainty in the linear homoskedastic regression model in finite samples.

It turns out that the GMM setting is well suited for incorporating parameter uncertainty in testing procedures by using Newey (1985) and Tauchen (1985). In this paper, we show that some testing functions are robust to the parameter uncertainty problem, that is the distribution of the test statistic when one uses the true unknown parameter coincides with the feasible test statistic when one uses an estimator of the parameter. Hermite polynomials are special examples of functions that have this robustness property. This result is a generalization of Kiefer and Salmon (1983) who showed that tests using Hermite polynomials are robust to parameter uncertainty when one considers a non linear homoskedastic regression estimated by the maximum likelihood method. In contrast, our result holds whatever the model and the estimation method. This property is very important when one uses advanced technical methods as in the stochastic volatility case. This result is the main contribution of the paper.

The second reason for using GMM is that when the variable of interest is serially correlated, the GMM setting is also well suited to take into account this dependence by using Heteroskedastic-Autocorrelation-Consistent (HAC) method of Newey and West (1987) and Andrews (1991). Using a HAC procedure in testing marginal distributions was already adopted by Richardson and Smith (1993) and Bai and Ng (2001) for testing normality, Ait-Sahalia (1996) and Conley, Hansen, Luttmer and Scheinkman (1997) for testing marginal distributions of non linear scalar diffusion processes.

\footnotetext{
${ }^{2}$ Hansen and Scheinkman (1995) gave two class of moment conditions related respectively to the marginal and conditional distributions of the process. Note, however, that while Hansen and Scheinkman (1995) derived these moment conditions in a Markovian case, we do not make this assumption.
} 
The paper is organized as follows. In section 2, we introduce the Stein equation and characterize its relationships with Hermite polynomials and Hansen and Scheinkman (1995) moment conditions. In section 3, we derive the test statistics we consider in both cross-sectional and time series cases. Then, we study the parameter uncertainty problem in section 4 . In section 5, we provide an extensive Monte Carlo study in order to assess the finite sample properties of the test statistics we consider and to compare them with the most popular methods, i.e. the Kolmogorov-Smirnov and Jarque-Bera tests. Section 6 applies our theory to three examples from the volatility literature while the last section concludes. All the proofs are provided in the Appendix.

\section{The Stein equation}

In this section, we first introduce the Stein (1972) equation which will be the basis of the test functions we consider to test normality. Then we specify this equation when one considers the Hermite polynomials. This is important because the most popular normality test in the econometric literature, namely the Jarque and Bera (1980) test, is based on moment conditions on the third and fourth Hermite polynomials. Finally, we also relate the Stein equation to the first moment conditions derived by Hansen and Scheinkman (1995) in the case of a continuous time process.

\subsection{The Stein equation}

Stein (1972) shows that a random variable $X$ has a standard normal distribution $\mathcal{N}(0,1)$ if and only if, for any differentiable function $f$ such that $E\left|f^{\prime}(Z)\right|<+\infty$ where $Z$ is $\mathcal{N}(0,1){ }^{3}$ we have

$$
E\left[f^{\prime}(X)-X f(X)\right]=0
$$

It is straightforward to show that (2.1) holds under normality. Hence, the main result of Stein (1972) is that (2.1) characterizes the normal distribution. The Stein equation (2.1) has several implications. A simple one is the classical recursive moment formula. More precisely, if one considers the case of monomial functions, that is $f_{i}(X)=X^{i}, i=0,1, \ldots$, the Stein equation (2.1) implies that

$$
E\left[X^{i+1}\right]=i E\left[X^{i-1}\right]
$$

The Stein equation (2.1) is the basic test function we consider for testing normality. This may be applied to monomials, polynomials and more general functions. An important property in the Stein equation is that by construction, the expectation of the considered function is zero.

\footnotetext{
${ }^{3}$ Observe that in the normal case, we have $E|X f(X)|<+\infty$ when $E\left|f^{\prime}(X)\right|<+\infty$.
} 
Therefore, one has not to compute theoretical formula as one would do when using for instance marginal moments. In other words, if one considers an integrable function $g$ and wants to check that the empirical counterpart of $\mathrm{E}[\mathrm{g}(\mathrm{X})]$ is close to the theoretical formula, then the Stein equation is useful because it is not necessary to compute the theoretical formula. Instead, one must compare the empirical counterparts of $E[g(X)]$ and $E[X G(X)]$ where $G$ is any primitive function of $g$. Of course, this means that one has to compute the primitive of $g$. An alternative solution is to define, when it is possible, the function $h(X) \equiv g(X) / X$. In this case, if one can use the function $h$ in the Stein equation, then one gets

$$
E[g(X)]=E[X h(X)]=E\left[h^{\prime}(X)\right] .
$$

There are some functions of interest for which the Stein equation becomes simple. This is the case for the Hermite polynomials we consider below.

\subsection{Hermite polynomials}

The orthonormalized Hermite polynomials $H_{i}$ associated to the $\mathcal{N}(0,1)$ distribution are defined by

$$
H_{i}(x)=\exp \left(\frac{x^{2}}{2}\right) \frac{(-1)^{i}}{\sqrt{i !}} \frac{d^{i} \exp \left(-x^{2} / 2\right)}{d x^{i}} .
$$

From (2.2), it is easy to show that the Hermite polynomials are given by the following recursive formula

$$
\forall i>1, H_{i}(x)=\frac{1}{\sqrt{i}}\left\{x H_{i-1}(x)-\sqrt{i-1} H_{i-2}(x)\right\}, H_{0}(x)=1, H_{1}(x)=x .
$$

By applying (2.3), we have

$$
H_{2}(x)=\frac{1}{\sqrt{2}}\left(x^{2}-1\right) ; H_{3}(x)=\frac{1}{\sqrt{6}}\left(x^{3}-3 x\right) ; H_{4}(x)=\frac{1}{\sqrt{24}}\left(x^{4}-6 x^{2}+3\right) .
$$

When a random variable $X$ follows a normal distribution $\mathcal{N}(0,1)$, the transformed random variables $H_{i}(X), i=0,1, \ldots$, have some interesting properties. In particular, they are orthonormal, that is:

$$
E\left[H_{i}(X) H_{j}(X)\right]=\delta_{i j}
$$

where $\delta_{i j}$ is the Kronecker symbol. By applying (2.5) to $j=0$ and $i \neq 0$, one gets

$$
\forall i>0, E\left[H_{i}(X)\right]=0,
$$

that is, the Hermite polynomials $H_{i}(X)$ are centered for $i>0$. 
In order to characterize the relationships between the Stein equation (2.1) and the Hermite polynomials, note that (2.2) implies the following restrictions are fulfilled by the derivatives of the Hermite polynomials:

$$
\begin{gathered}
H_{i}^{\prime}(x)=\sqrt{i} H_{i-1}(x) \text { and } \\
H_{i}^{\prime \prime}(x)-x H_{i}^{\prime}(x)+i H_{i}(x)=0 .
\end{gathered}
$$

Let us now apply the Stein equation $(2.1)$ to the function $H_{i}^{\prime}(x) / \sqrt{i}$. This function is clearly differentiable and integrable. Therefore, we have

$$
\frac{1}{\sqrt{i}} E\left[H_{i}^{\prime \prime}(X)-X H_{i}^{\prime}(X)\right]=0 .
$$

By using (2.8), this equation implies (2.6). As a consequence, the Stein equation (2.1) implies (2.6). It turns out that the converse holds also:

Proposition 2.1 Let $X$ be a random variable such that $\forall i>0, E\left[H_{i}(X)\right]=0$. Then, the equation (2.1) holds for any differentiable function $f$ such that $E\left[\left|f^{\prime}(Z)\right|\right]<+\infty$ where $Z$ is assumed to be $\mathcal{N}(0,1)$.

As a consequence, a random variable $X$ is $\mathcal{N}(0,1)$ if and only if (2.6) holds.

This means that for statistical inference purposes, in particular testing, one could use Hermite polynomials only.

\subsection{Continuous time case}

Consider a univariate diffusion process $X_{t}$ assumed to be the stationary solution of

$$
d X_{t}=\mu\left(X_{t}\right) d t+\sigma\left(X_{t}\right) d W_{t},
$$

where $W_{t}$ is a standard Brownian process. Then, Hansen and Scheinkman (1995) provide two sets of moment conditions related to the marginal and conditional distributions of $X_{t}$ respectively. For the marginal distribution, Hansen and Scheinkman (1995) show that

$$
E\left[\mathcal{A} g\left(X_{t}\right)\right]=0,
$$

where $g$ is assumed to be twice differentiable and square-integrable with respect to the marginal distribution of $X_{t}$ and $\mathcal{A}$ is the infinitesimal generator associated to the diffusion (2.9), that is:

$$
\mathcal{A} g(x)=\mu(x) g^{\prime}(x)+\frac{\sigma^{2}(x)}{2} g^{\prime \prime}(x) .
$$

A well known continuous time process for which the marginal distribution is $\mathcal{N}(0,1)$ is the standardized Ornstein-Uhlenbeck process defined by

$$
d X_{t}=-k X_{t} d t+\sqrt{2 k} d W_{t}, \quad k>0, \quad X_{0} \sim \mathcal{N}(0,1) .
$$


For this process, Hansen and Scheinkman (1995) moment condition (2.10) becomes:

$$
E\left[-k X_{t} g^{\prime}\left(X_{t}\right)+k g^{\prime \prime}\left(X_{t}\right)\right]=0 .
$$

Thus, by considering the function $f$ defined by $f \equiv g^{\prime}$, we obtain the Stein equation (2.1) (since $k \neq 0$ ). Thus, the Hansen and Scheinkman (1995) moment condition (2.10) coincides with the Stein equation (2.1).

The continuous time setting provides examples of processes where the marginal distribution is normal while the conditional distribution is not. A first example may be constructed as follows. For a given specification of $\mu(x)$ and $\sigma(x)$, the marginal density function of the process $X_{t}$ is, up to a scale, ${ }^{4}$

$$
\sigma(x)^{-2} \exp \left(\int_{z}^{x} \frac{2 \mu(u)}{\sigma(u)^{2}} d u\right) .
$$

This density function suggests that two different specifications of $\mu(x)$ and $\sigma(x)$ may give the same marginal distribution. It turns out that this is the case. ${ }^{5}$ As a consequence, it is possible to get a scalar diffusion such that the marginal distribution is $\mathcal{N}(0,1)$ while the conditional distribution is not normal, that is a non Ornstein-Uhlenbeck process.

A second example may be obtained by subordination. More precisely, assume that we observe a sample $x_{1}, x_{2}, \ldots, x_{T}$ of a process $X_{t}$ with $X_{t}=Y_{S_{t}}$, where $Y_{t}$ is a stationary scalar diffusion and $S_{t}, t=1, \ldots, T$, is a positive and increasing process with $S_{1}=1$. Under the assumption that the processes $\left\{Y_{\tau}, \tau \in \mathbb{R}^{+}\right\}$and $\left\{S_{t}, t \in \mathbf{N}^{*}\right\}$ are independent, the marginal distribution of the processes $X_{t}$ and $Y_{t}$ coincide. Therefore, if the process $Y_{t}$ is a standardized Ornstein-Uhlenbeck process, the marginal distribution of $X_{t}$ is $\mathcal{N}(0,1)$ while its conditional distribution is (in general) not normal.

\section{$3 \quad$ Test statistics}

In the section, we provide the test statistics for testing normality. All of them are based on the Stein equation (2.1). We study in detail the cross-sectional and the time series cases. In this section, we assume that we observe a sample of the random variable of interest, i.e., we do not take into account the potential problem of parameter uncertainty (see next section).

\subsection{The general case}

Consider a sample $x_{1}, \ldots, x_{T}$, of the variable of interest denoted by $X$. The observations may be independent or dependent. We assume that the marginal distribution of $X$ is $\mathcal{N}(0,1)$. Let $f_{1}$,

\footnotetext{
${ }^{4}$ For a given $\mathrm{z}$, the scale parameter is chosen so that the density integral equals one.

${ }^{5}$ See Ait-Sahalia, Hansen and Scheinkman (2001) for a review of all the properties of diffusion processes we consider in this paper.
} 
$\ldots, f_{p}$, be $p$ differentiable functions such that $f_{i}^{\prime}$ is integrable. For a real $x$, define the vector $g(x)$

$\in \mathbb{R}^{p}$, which components are $\left(f_{i}^{\prime}(x)-x f_{i}(x)\right)$ for $i=1, \ldots, p$. Thus, by the Stein equation (2.1), we have $E[g(X)]=0$. Assume that any component of the vector $g(X)$ is square-integrable and that the matrix $\Sigma$ defined by

$$
\Sigma \equiv \lim _{T \rightarrow+\infty} \operatorname{Var}\left[\frac{1}{\sqrt{T}} \sum_{t=1}^{T} g\left(x_{t}\right)\right]=\sum_{h=-\infty}^{+\infty} E\left[g\left(x_{t}\right) g\left(x_{t-h}\right)^{\top}\right],
$$

is finite and definite positive, then we have (see Hansen, 1982)

$$
\frac{1}{\sqrt{T}} \sum_{t=1}^{T} g\left(x_{t}\right) \longrightarrow \mathcal{N}(0, \Sigma)
$$

while

$$
\left(\frac{1}{\sqrt{T}} \sum_{t=1}^{T} g\left(x_{t}\right)\right)^{\top} \Sigma^{-1}\left(\frac{1}{\sqrt{T}} \sum_{t=1}^{T} g\left(x_{t}\right)\right) \sim \chi^{2}(p) .
$$

For the feasibility of the test procedure, one needs the matrix $\Sigma$ or at least a consistent estimator. It is clear that if one does not specify the dependence between the observations $x_{1}$, $x_{2}, \ldots, x_{T}$, then one needs to estimate $\Sigma$.

\subsection{The cross-sectional case}

Consider the cross-sectional case and assume that the observations are independent and identically distributed (i.i.d.). In this case, we have

$$
\Sigma=\operatorname{Var}[g(X)]=E\left[g(X) g(X)^{\top}\right] .
$$

Observe that by using the Stein equation (2.1), one can have a simple form for $E\left[g(X) g(X)^{\top}\right]$. More precisely, it is easy to show that

$$
E\left[g(X) g(X)^{\top}\right]=E\left[f(X) f(X)^{\top}+f^{\prime}(X) f^{\prime}(X)^{\top}\right] .
$$

Two cases may arise. In the first one, one can explicitly compute the matrix $\Sigma$ and, hence, one can use the test statistic (3.3). This is the case for the Hermite polynomials that we consider below. In the second case, computing explicitly $\Sigma$ is not possible (or difficult), then one can use any consistent estimator of $\Sigma$ and denoted by $\hat{\Sigma}_{T}$. Examples of consistent estimators of $\Sigma_{T}$ are

$$
\hat{\Sigma}_{1, T}=\frac{1}{T} \sum_{t=1}^{T} g\left(x_{t}\right) g\left(x_{t}\right)^{\top} \text { and } \hat{\Sigma}_{2, T}=\frac{1}{T} \sum_{t=1}^{T}\left(f\left(x_{t}\right) f\left(x_{t}\right)^{\top}+f^{\prime}\left(x_{t}\right) f^{\prime}\left(x_{t}\right)^{\top}\right) .
$$


In this case, one can use the following test statistic

$$
\left(\frac{1}{\sqrt{T}} \sum_{t=1}^{T} g\left(x_{t}\right)\right)^{\top} \hat{\Sigma}_{T}^{-1}\left(\frac{1}{\sqrt{T}} \sum_{t=1}^{T} g\left(x_{t}\right)\right) \sim \chi^{2}(p) .
$$

Assume now that we consider the Hermite polynomials. We showed in the previous section that when one applies the Stein equation (2.1) to the function $f_{i}(x)=H_{i+1}^{\prime}(x) / \sqrt{i}$, one gets

$$
E\left[H_{i}(X)\right]=0 .
$$

But the unconditional variance of $H_{i}(x)$ is one. Hence, for $i \geq 0$, we have

$$
\frac{1}{\sqrt{T}} \sum_{t=1}^{T} H_{i}\left(x_{t}\right) \longrightarrow \mathcal{N}(0,1) \text { and }\left(\frac{1}{\sqrt{T}} \sum_{t=1}^{T} H_{i}\left(x_{t}\right)\right)^{2} \sim \chi^{2}(1) .
$$

Moreover, the Hermite polynomials are orthogonal. Hence, the test statistic based on different Hermite polynomials are asymptotically independent. In other words, when one uses a test statistic based on several Hermite polynomials, the corresponding matrix $\Sigma$ derived previously is diagonal. Besides, the diagonal matrix $\Sigma$ is indeed the identity since the variance of each Hermite polynomial equals one. For instance, if we consider the Hermite polynomials $H_{3}, H_{4}, \ldots, H_{p}$, then the test statistic is

$$
\sum_{i=3}^{p}\left(\frac{1}{\sqrt{T}} \sum_{t=1}^{T} H_{i}\left(x_{t}\right)\right)^{2} \sim \chi^{2}(p-2) .
$$

It is worth noting that this result is more general than one of Kiefer and Salmon (1982) who showed (3.8) when the variables $x_{t}$ are estimated residuals in a linear model when one uses the maximum likelihood method. We will discuss Kiefer and Salmon (1982) results in more detail in the next section where we consider the parameter uncertainty problem.

\subsection{The time series case}

Assume now that the observations are correlated and represent a sample of a process. Then, without additional assumptions on the dependence, one can not explicitly compute the matrix $\Sigma$ and has to estimate it. A traditional solution is to estimate this matrix by using a Heteroskedastic-Autocorrelation-Consistent (HAC) method like Newey and West (1987) or Andrews (1991). This is one of the motivations of using a GMM approach for testing normality. This was already used by Smith and Richardson (1991) and was more recently and independently of our work highlighted by Bai and Ng (2001).

In contrast to the cross-sectional case, one can not show that test statistics based on two different Hermite polynomials are asymptotically independent. More precisely, consider a component $(i, j)$, with $i \neq j$, of the matrix $\Sigma$. In this case, $E\left[H_{i}\left(x_{t}\right) H_{j}\left(x_{t}\right)\right]$ is zero by the 
orthogonality of the Hermite polynomials (2.5). However, without additional restrictions, we think that $E\left[H_{i}\left(x_{t}\right) H_{j}\left(x_{t-h}\right)\right]$ is in general nonzero for $h \neq 0$. Thus, the matrix $\Sigma$ will be in general non diagonal. ${ }^{6}$

In contrast, this may be the case if one makes additional assumptions on the dependence of the process $x_{t}$. An important example is when one assumes that the process $x_{t}$ is a normal autoregressive process of order one, $\operatorname{AR}(1)$, that is

$$
x_{t}=\gamma x_{t-1}+\sqrt{1-\gamma^{2}} \varepsilon_{t}, \quad \varepsilon_{t} \text { i.i.d. } \mathcal{N}(0,1) \text { and }|\gamma|<1 .
$$

In this case, any Hermite polynomial $H_{i}\left(x_{t}\right)$ is an AR(1) process which autoregressive coefficient equals $\gamma^{i}$, that is

$$
E\left[H_{i}\left(x_{t+1}\right) \mid x_{\tau}, \tau \leq t\right]=\gamma^{i} H_{i}\left(x_{t}\right) .
$$

In this case, it is easy to show that

$$
\Sigma_{i j}=\sum_{h=-\infty}^{+\infty} E\left[H_{i}\left(x_{t}\right) H_{j}\left(x_{t-h}\right)\right]=\frac{1+\gamma^{i}}{1-\gamma^{i}} \delta_{i j} .
$$

As a consequence, the matrix $\Sigma$ is diagonal and, hence, the test statistics based on different Hermite polynomials are asymptotically independent. Besides, when one tests normality and ignores the dependence of the Hermite polynomials, one gets a wrong distribution for the test statistic. For instance, assume that one considers a test based on a particular Hermite polynomial $H_{i}$. Then, the test statistic becomes

$$
\frac{1-\gamma^{i}}{1+\gamma^{i}}\left(\frac{1}{\sqrt{T}} \sum_{t=1}^{T} H_{i}\left(x_{t}\right)\right)^{2} \sim \chi^{2}(1) .
$$

Thus, by ignoring the dependence of the Hermite polynomial $H_{i}\left(x_{t}\right)$, one overrejects the normality when $\gamma \geq 0$ or $i$ is even and underrejects otherwise. Monte Carlo simulations in the sixth section will assess this. This is important in practice since many economic time series are positively autocorrelated.

\subsection{Skewness and excess kurtosis}

A traditional approach for testing normality is to study the skewness and excess kurtosis of the variable of interest (Mardia, 1970; Bowman and Shenton, 1975; Jarque and Bera, 1980). More precisely, when a random variable $X$ is distributed as $\mathcal{N}\left(0, \sigma^{2}\right)$, we have

$$
E\left[X^{3}\right]=0 \text { and } E\left[X^{4}-3 \sigma^{4}\right]=0,
$$

\footnotetext{
${ }^{6}$ In their paper, Bai and $\mathrm{Ng}(2001)$ write in page 5 that the tests based on the excess skwness and excess kurtosis are asymptotically independent under normality even for time series data. They do not provide a proof however.
} 
where the first condition deals with skewness while the second one deals with excess kurtosis. For simplicity, assume that we observe an i.i.d. sample $x_{1}, x_{2}, \ldots, x_{T}$. Then, when the parameter $\sigma^{2}$ is known, the test statistic implied by moment condition in (3.13) is

$$
\sqrt{T}\left(\begin{array}{c}
\frac{1}{T} \sum_{t=1}^{T} x_{t}^{3} \\
\frac{1}{T} \sum_{t=1}^{T} x_{t}^{4}-3 \sigma^{4}
\end{array}\right) \stackrel{n \rightarrow+\infty}{\longrightarrow} N\left(\begin{array}{c}
0 \\
0
\end{array},\left(\begin{array}{cc}
15 \sigma^{6} & 0 \\
0 & 96 \sigma^{8}
\end{array}\right)\right) .
$$

Thus, skewness and excess kurtosis test statistics are asymptotically independent. Moreover, we have the following test statistic

$$
\frac{1}{15}\left(\frac{1}{\sqrt{T}} \sum_{t=1}^{T}\left(x_{t} / \sigma\right)^{3}\right)^{2}+\frac{1}{96}\left(\frac{1}{\sqrt{T}} \sum_{t=1}^{T}\left[\left(x_{t} / \sigma\right)^{4}-3\right]\right)^{2} \stackrel{n \rightarrow+\infty}{\longrightarrow} \chi^{2}(2) .
$$

Note that this test statistic is different from one of Jarque and Bera (1980) given by

$$
\frac{1}{6}\left(\frac{1}{\sqrt{T}} \sum_{t=1}^{T}\left(x_{t} / \hat{\sigma}\right)^{3}\right)^{2}+\frac{1}{24}\left(\frac{1}{\sqrt{T}} \sum_{t=1}^{T}\left[\left(x_{t} / \hat{\sigma}\right)^{4}-3\right]\right)^{2} \stackrel{n \rightarrow+\infty}{\longrightarrow} \chi^{2}(2),
$$

where $\hat{\sigma}$ is the MLE of $\sigma$ in a regression model. This difference is due to parameter uncertainty that we consider in the following section.

\subsection{OPG regression}

Davidson and MacKinnon (1993) considered testing normality through skewness and excess kurtosis. In particular, they reformulated Jarque and Bera (1980) test in terms of outerproduct-of-the-gradient (OPG) regression.

More precisely, as in Davidson and MacKinnon (1993), assume that one is interested in testing that $y_{t}$ is $\mathcal{N}\left(\beta, \sigma^{2}\right)$ where $\sigma^{2}$ is a known parameter and define $x_{t}$ by $x_{t}=y_{t}-\beta$. The assumption that the mean and variance of $x_{t}$ are zero and $\sigma^{2}$ could be tested by the following OPG regression

$$
1=b x_{t}+s\left(x_{t}^{2}-\sigma^{2}\right)+\text { residual }
$$

Assume now that one is interested in testing skewness. Then, Davidson and MacKinnon (1993) propose to add in (3.17) the regressor $x_{t}^{3}$, i.e.,

$$
1=b x_{t}+s\left(x_{t}^{2}-\sigma^{2}\right)+a x_{t}^{3}+\text { residual, }
$$

and to test that the coefficient $a$ is zero. The test statistic being the $t$ statistic of the estimator of $a$. Given that $x_{t}^{3}$ is orthogonal with $x_{t}^{2}-\sigma^{2}$ and not with $x_{t}$, the numerator of the $t$ test is the mean of $x_{t}^{3}$ minus the mean of its projection on $x_{t}$, i.e.

$$
\frac{1}{T} \sum_{t=1}^{T} x_{t}^{3}-\left(\frac{\sum_{t=1}^{T} x_{t}^{4}}{\sum_{t=1}^{T} x_{t}^{2}}\right) \frac{1}{T} \sum_{t=1}^{T} x_{t}
$$


which is, under normality and when $T$ is large, very close to

$$
\frac{1}{T} \sum_{t=1}^{T} x_{t}^{3}-3 \sigma^{2} \frac{1}{T} \sum_{t=1}^{T} x_{t} .
$$

Davidson and MacKinnon (1993) showed that the variance of $x_{t}^{3}-3 \sigma^{2} x_{t}$ is $6 \sigma^{6}$. Thus, the $t$ statistic is very close to

$$
\frac{\frac{1}{T} \sum_{t=1}^{T} x_{t}^{3}-3 \sigma^{2} \frac{1}{T} \sum_{t=1}^{T} x_{t}}{\sqrt{6 \sigma^{6}}} .
$$

Similarly, Davidson and MacKinnon (1993) showed that one can test excess kurtosis by doing the following OPG regression

$$
1=b x_{t}+s\left(x_{t}^{2}-\sigma^{2}\right)+a x_{t}^{3}+b\left(x_{t}^{4}-3 \sigma^{4}\right)+\text { residual, }
$$

and that the t-statistic related to the coefficient $b$ is very close to

$$
\frac{\frac{1}{T} \sum_{t=1}^{T} x_{t}^{4}-6 \sigma^{2} \frac{1}{T} \sum_{t=1}^{T} x_{t}^{2}+3 \sigma^{4}}{\sqrt{24 \sigma^{8}}} .
$$

Observe that the test statistics (3.19) and (3.21) may be written as

$$
\frac{1}{T} \sum_{t=1}^{T} \frac{1}{\sqrt{6}}\left[\left(x_{t} / \sigma\right)^{3}-3\left(x_{t} / \sigma\right)\right]=\frac{1}{T} \sum_{t=1}^{T} H_{3}\left(x_{t} / \sigma\right)
$$

and

$$
\frac{1}{T} \sum_{t=1}^{T} \frac{1}{\sqrt{24}}\left[\left(x_{t} / \sigma\right)^{4}-6\left(x_{t} / \sigma\right)^{2}+3\right]=\frac{1}{T} \sum_{t=1}^{T} H_{4}\left(x_{t} / \sigma\right) .
$$

Given that the variance of $H_{3}\left(x_{t} / \sigma\right)$ is one, the test statistic (3.19) is also the $t$-statistic of the coefficient $\tilde{a}$ in the two OPG regressions

$$
\begin{gathered}
1=b x_{t}+s\left(x_{t}^{2}-\sigma^{2}\right)+\tilde{a} H_{3}\left(x_{t} / \sigma\right)+\text { residual, and } \\
1=\tilde{b} H_{1}\left(x_{t} / \sigma\right)+\tilde{s} H_{2}\left(x_{t} / \sigma\right)+\tilde{a} H_{3}\left(x_{t} / \sigma\right)+\text { residual. }
\end{gathered}
$$

In addition, given that the variance of $H_{4}\left(x_{t} / \sigma\right)$ is one, the test statistic (3.21) is also the $t$-statistic of the coefficient $\tilde{b}$ in the two OPG regressions

$$
\begin{gathered}
1=b x_{t}+s\left(x_{t}^{2}-\sigma^{2}\right)+\tilde{b} H_{4}\left(x_{t} / \sigma\right)+\text { residual, and } \\
1=\tilde{b} H_{1}\left(x_{t} / \sigma\right)+\tilde{s} H_{2}\left(x_{t} / \sigma\right)+\tilde{b} H_{4}\left(x_{t} / \sigma\right)+\text { residual. }
\end{gathered}
$$


Finally, because $H_{3}\left(x_{t} / \sigma\right)$ and $H_{4}\left(x_{t} / \sigma\right)$ are orthogonal, the test statistics (3.19) and (3.21) are respectively the $t$-statistic of the coefficients $\tilde{a}$ and $\tilde{b}$ in the two OPG regressions

$$
\begin{gathered}
1=b x_{t}+s\left(x_{t}^{2}-\sigma^{2}\right)+\tilde{a} H_{3}\left(x_{t} / \sigma\right)+\tilde{b} H_{4}\left(x_{t} / \sigma\right)+\text { residual, and } \\
1=\tilde{b} H_{1}\left(x_{t} / \sigma\right)+\tilde{s} H_{2}\left(x_{t} / \sigma\right)+\tilde{a} H_{3}\left(x_{t} / \sigma\right)+\tilde{b} H_{4}\left(x_{t} / \sigma\right)+\text { residual. }
\end{gathered}
$$

In other words, given the orthonormality of Hermite polynomials, testing that the empirical mean of the third Hermite polynomial is numerically the same as testing that the coefficient $\tilde{a}$ in the previous regressions is zero. It is also the case when one tests excess kurtosis by using the fourth Hermite polynomial and, more generally, for higher order Hermite polynomials.

\section{Parameter uncertainty}

In most empirical examples, the normality assumption is made for an unobservable random variable. This is the case for a regression, linear or non linear, homoskedastic or heteroskedastic, where the normality assumption is in general made on the (standardized) residuals. This is also the case for non linear time series as volatility models (e.g., GARCH or stochastic volatility models). Thus, one must first estimate the parameters of the model and then get fitted residuals. Then, one tests the normality assumption of the residuals by using the fitted residuals. In some other empirical examples, the normality assumption is made on observable variables but the parameter of the normal distribution, i.e., the mean and the variance, are unknown. Therefore, one must also estimate these parameters in order to test normality.

It is well known that the asymptotic distribution of a test statistic that depends on an unknown parameter, denoted by $\theta^{0}$, may be different from the asymptotic distribution of the same test statistic applied by using a consistent estimator of $\theta^{0}$, denoted by $\hat{\theta}_{T}$. The main reason is that one has to take into account the uncertainty of $\hat{\theta}_{T}$ in the testing procedure. This is known as the parameter uncertainty problem.

The GMM approach is well suited for this problem, which is the first reason we are using it in the paper to test normality. Newey (1985) and Tauchen (1985) provided a general theory for taking into account the parameter uncertainty in testing procedures. Their approach is the following. Assume that one has to test the following moment condition

$$
E\left[g\left(z_{t}, \theta^{0}\right)\right]=0,
$$

where $z_{t}$ is a random variable, potentially multivariate, and $\theta^{0}$ is an unknown (vectorial) parameter. Under the null hypothesis, we have

$$
\frac{1}{\sqrt{T}} \sum_{t=1}^{T} g\left(z_{t}, \theta^{0}\right) \rightarrow \mathcal{N}\left(0, \Sigma_{g}\right) \text { where } \Sigma_{g}=\lim _{T \rightarrow+\infty} \operatorname{Var}\left[\frac{1}{\sqrt{T}} \sum_{t=1}^{T} g\left(z_{t}, \theta^{0}\right)\right] .
$$


Assume that one has a square-root $T$ consistent estimator of $\theta^{0}$, denoted by $\hat{\theta}_{T}$, i.e.

$$
\sqrt{T}\left(\hat{\theta}_{T}-\theta^{0}\right) \rightarrow \mathcal{N}\left(0, V_{\theta}\right) .
$$

Then, a natural approach to test (4.1) is by using $T^{-1 / 2} \sum_{t=1}^{T} g\left(z_{t}, \hat{\theta}_{T}\right)$. Therefore, one needs the asymptotic distribution of this test statistic. It is easily obtained by using a Taylor approximation around the unknown parameter $\theta^{0}$. More precisely, we have:

$$
\frac{1}{\sqrt{T}} \sum_{t=1}^{T} g\left(z_{t}, \hat{\theta}_{T}\right)=\frac{1}{\sqrt{T}} \sum_{t=1}^{T} g\left(z_{t}, \theta^{0}\right)+\left[\frac{1}{T} \sum_{t=1}^{T} \frac{\partial g\left(z_{t}, \theta^{0}\right)}{\partial \theta^{\top}}\right] \sqrt{T}\left(\hat{\theta}_{T}-\theta^{0}\right)+o_{p}(1) .
$$

Define the matrix $P_{g}$ by

$$
P_{g}=\lim _{T \rightarrow+\infty} \frac{1}{T} \sum_{t=1}^{T} \frac{\partial g\left(z_{t}, \theta^{0}\right)}{\partial \theta^{\top}} .
$$

Then, we can rewrite (4.4) in the following form:

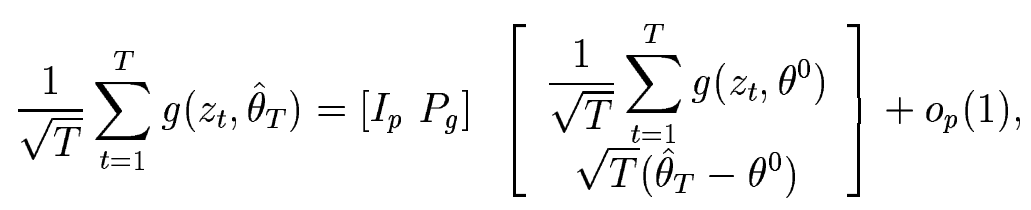

where $I_{p}$ is the $p \times p$ identity matrix and $p$ the dimension of $g$. From (4.6), it is clear that the asymptotic distribution of the test statistic in the left hand side of (4.6) depends on the asymptotic distributions of two random variables, $T^{-1 / 2} \sum_{t=1}^{T} g\left(z_{t}, \theta^{0}\right)$ and $\sqrt{T}\left(\hat{\theta}_{T}-\theta^{0}\right)$ which are given respectively in (4.2) and (4.3), and their asymptotic covariance. ${ }^{7}$ As a consequence, the parameter uncertainty generally changes the asymptotic distribution of test statistic when one uses an estimator instead of the unknown parameter $\theta^{0}$.

In general, the matrices that appear in the asymptotic distributions (4.2) and (4.3) are easily estimated. However, it is difficult is to estimate the asymptotic covariance matrix between $T^{-1 / 2} \sum_{t=1}^{T} g\left(z_{t}, \theta^{0}\right)$ and $\sqrt{T}\left(\hat{\theta}_{T}-\theta^{0}\right)$. This is the case when one uses advanced estimation methods, especially simulation techniques ${ }^{8}$ as in the case of stochastic volatility and latent factor models.

An alternative method that adopted in this paper is to consider moment conditions such that the matrix $P_{g}$ is zero, i.e.,

$$
P_{g}=0
$$

In this case, the asymptotic distribution of $T^{-1 / 2} \sum_{t=1}^{T} g\left(z_{t}, \theta^{0}\right)$ and $T^{-1 / 2} \sum_{t=1}^{T} g\left(z_{t}, \hat{\theta}_{T}\right)$ coincide. Hence, the test statistic is robust against the parameter uncertainty.

\footnotetext{
${ }^{7}$ We assume that the asymptotic distribution of the right hand side of (4.6) is normal.

${ }^{8}$ See Gouriéroux and Monfort (1996) for a review.
} 
In the sequel, we need to be more specific about the examples we will consider in order to characterize the moment conditions that are robust against the parameter uncertainty. We consider three examples:

Example 1: regression with exogenous variables. Let $z_{t}=\left(y_{t}, x_{t}^{\top}\right)^{\top}$ be a vector where $y_{t}$ is an endogenous variable, $x_{t}$ is a (vectorial) exogenous variable. We assume that there exists a unique parameter $\theta^{0}=\left(\alpha^{0 \top}, \beta^{0 \top}\right)^{\top}$ such that

$$
y_{t}=m\left(x_{t}, \beta^{0}\right)+\sigma\left(x_{t}, \beta^{0}, \alpha^{0}\right) u_{t} \text {, and } u_{t} \mathcal{N}(0,1),
$$

where $\alpha^{0}$ and $\beta^{0}$ are real vectors and $m(x, \beta)$ and $\sigma(x, \beta, \alpha)$ are two real functions. A special example is the cross-sectional case where the random variable $u_{t}$ is i.i.d. by assumption. Another example is the time series case where the variable $u_{t}$ may be serially correlated. However, it is assumed to be independent from $x_{t}$.

The model adopted by Jarque and Bera (1980) is the special case where

$$
m\left(x_{t}, \beta\right)=x_{t}^{\top} \beta \text { and } \sigma\left(x_{t}, \beta, \alpha\right)=\alpha,
$$

i.e., they considered a linear homoskedastic regression model with potentially correlated residuals. Kiefer and Salmon (1983) adopted also a special case of (4.8) by assuming

$$
\sigma\left(x_{t}, \beta, \alpha\right)=\alpha \text { and } u_{t} \text { i.i.d., }
$$

i.e., a non linear regression model with homoskedastic and i.i.d. errors.

Example 2: time series regression. This example is similar to the first one, but we now assume that the variables $x_{t}$ are lagged values of $y_{t}$ and $u_{t}$ is i.i.d., i.e., ${ }^{9}$

$$
\begin{gathered}
E\left[y_{t} \mid y_{\tau}, \tau \leq t-1\right]=m_{t}\left(\beta^{0}\right), \operatorname{Var}\left[y_{t} \mid y_{\tau}, \tau \leq t-1\right]=\sigma_{t}^{2}\left(\beta^{0}, \alpha^{0}\right), \\
u_{t} \equiv \frac{y_{t}-m_{t}\left(\beta^{0}\right)}{\sigma_{t}\left(\alpha^{0}\right)} \text { and } u_{t} \text { i.i.d. } \mathcal{N}(0,1) .
\end{gathered}
$$

Special examples of this case are ARMA models with GARCH errors (Bollerslev, 1986).

Example 3: marginal distribution of a process. In this case, we assume that we observe a sample $y_{1}, \ldots, y_{T}$, of a process which marginal distribution is assumed to be $\mathcal{N}\left(m^{0}, \sigma^{02}\right)$ where $m^{0}$ and $\sigma^{0}$ are unknown parameters. Hence, the standardized process is $\mathcal{N}(0,1)$, that is

$$
u_{t} \equiv \frac{y_{t}-m^{0}}{\sigma^{0}} \text { and } u_{t} \mathcal{N}(0,1) .
$$

Observe that in all these examples, the normal variable of interest $u_{t}$ may be written as

$$
u_{t}(\theta)=\frac{y_{t}-m_{t}(\theta)}{\sigma_{t}(\theta)},
$$

where the normality assumption holds for $u_{t}\left(\theta^{0}\right)$ and denoted by $u_{t}$. We can now characterize the test functions $g$ that are robust against parameter uncertainty in Example 1, 2 or 3 .

\footnotetext{
${ }^{9}$ Observe that we adopt a different notation than for the first example in order to incorporate non Markovian models like MA and GARCH.
} 
Proposition 4.1 Consider $u_{t}$ as defined in Example 1, 2 or 3. Let $\hat{\theta}_{T}$ be a square-root $T$ consistent estimator of $\theta^{0}$ such that (4.3) and $\hat{u}_{t}$ the corresponding estimated residuals. Define the function $\tilde{g}($.$) by \tilde{g}\left(u_{t}(\theta)\right)=g\left(z_{t}, \theta\right)$. Then, a sufficient condition such that the asymptotic distribution of the test statistics $T^{-1 / 2} \sum_{t=1}^{T} g\left(z_{t}, \theta^{0}\right)$ and $T^{-1 / 2} \sum_{t=1}^{T} g\left(z_{t}, \hat{\theta}_{T}\right)$ coincide is

$$
E\left[\tilde{g}^{\prime}\left(u_{t}\right)\right]=0 \text { and } E\left[u_{t} \tilde{g}^{\prime}\left(u_{t}\right)\right]=0
$$

This proposition means that a sufficient conditions ensuring the robustness of our test statistic against the parameter uncertainty is the orthogonality of $\tilde{g}^{\prime}$ with $H_{0}$ and $H_{1}$, i.e.

$$
E\left[H_{0}\left(u_{t}\right) \tilde{g}^{\prime}\left(u_{t}\right)\right]=0 \text { and } E\left[H_{1}\left(u_{t}\right) \tilde{g}^{\prime}\left(u_{t}\right)\right]=0
$$

It is worth noting that we do not assume that the considered test statistic comes from the Stein equation (2.1). Indeed, this result encompasses the results of White and MacDonald (1980). Besides, this proposition holds in both cross-sectional and time series cases. Finally, while (4.15) is a sufficient condition, it is generically necessary. It may not be necessary for some estimators with very particular asymptotic variances.

Before further characterizing (4.16) when one considers the Stein equation (2.1), let us apply this proposition when one considers tests based on excess skewness and kurtosis as did Jarque and Bera (1980) and Bai and Ng (2001). More precisely, assume that one considers the moment conditions

$$
E\left[\tilde{g}_{1}\left(u_{t}\right)\right]=0 \text { and/or } E\left[\tilde{g}_{2}\left(u_{t}\right)\right]=0 \text {, where } \tilde{g}_{1}\left(u_{t}\right)=u_{t}^{3} \text { and } \tilde{g}_{2}\left(u_{t}\right)=u_{t}^{4}-3 \text {. }
$$

It is clear that the function $\tilde{g}_{1}$ violates the first condition in (4.15) while $\tilde{g}_{2}$ violates the second one. Thus, in this case, one must correct the asymptotic distribution of the test statistic by taking into account the parameter uncertainty. Jarque and Bera (1980) did this correction by using a Lagrange multiplier test.

When one considers test statistics based on the Stein equation (2.1), that is when one assumes that

$$
\tilde{g}(x)=f^{\prime}(x)-x f(x)
$$

the condition (4.15) may be characterized through the function $f(x)$ :

Proposition 4.2 Let $f(x)$ be a differentiable function and define $\tilde{g}(x)$ by $\tilde{g}(x) \equiv f^{\prime}(x)-x f(x)$. Then, the condition (4.16) holds if and only if

$$
E\left[f\left(u_{t}\right)\right]=0 \text { and } E\left[f^{\prime}\left(u_{t}\right)\right]=0 .
$$

This proposition may be easily applied in practice. One has to take any integrable function denoted by $s(x)$ such that $E[|s(Z)|]<+\infty$ where $Z$ is assumed to be $\mathcal{N}(0,1)$. Then, define 
the function $\bar{s}(x)$ by $\bar{s}(x)=s(x)-E[s(Z)]$ and the function $\bar{f}(x)$ as the primitive of $\bar{s}(x)$ which is centered, that is $E[\bar{f}(Z)]=0$. Then, by construction, the condition (4.18) holds for $\bar{f}(x)$.

When on uses the conditions based on the Hermite polynomials (2.6), the conditions (4.15) and (4.18) hold for any (linear combination of) Hermite polynomial $H_{i}(x)$ with $i \geq 3$. This is the main result of our paper:

Proposition 4.3 Consider $u_{t}$ as defined in Example 1, 2 or 3. Let $\hat{\theta}_{T}$ be a square-root $T$ consistent estimator of $\theta^{0}$ such that (4.3). Let $g$ be a vectorial function such that any component is a linear combination of Hermite polynomials $H_{i}(x)$ with $i \geq 3$. Then, the asymptotic distribution of the test statistics $T^{-1 / 2} \sum_{t=1}^{T} g\left(z_{t}, \theta^{0}\right)$ and $T^{-1 / 2} \sum_{t=1}^{T} g\left(z_{t}, \hat{\theta}_{T}\right)$ coincide.

This result was already stated in Kiefer and Salmon (1983). However these authors assumed that the model is a non linear regression with homoskedastic and i.i.d. errors, that is under (4.8) and (4.10). Moreover, they showed this result when $\hat{\theta}_{T}$ is the maximum likelihood estimator. In other words, both assumptions are relaxed in the previous proposition. This is very important in many empirical examples where computing the maximum likelihood estimator is difficult or unfeasible. In addition, the results of Davidson and MacKinnon (1993) in their OPG regression where the consider parameter uncertainty are also a special case of the previous proposition.

We now characterize the relationship of the Jarque and Bera (1980) test with the previous proposition. The test statistic they proposed is for example one under (4.9). More precisely, let $\hat{u}_{t}$ defined by

$$
\hat{u}_{t}=\frac{y_{t}-x_{t}^{\top} \hat{\beta}}{\hat{\alpha}}
$$

where $\hat{\alpha}$ and $\hat{\beta}$ are the MLE of $\alpha$ and $\beta$. Then, Jarque and Bera (1980) showed that

$$
\frac{1}{6}\left(\frac{1}{\sqrt{T}} \sum_{t=1}^{T} \hat{u}_{t}^{3}\right)^{2}+\frac{1}{24}\left(\frac{1}{\sqrt{T}} \sum_{t=1}^{T}\left[\hat{u}_{t}^{4}-3\right]\right)^{2} \stackrel{n \rightarrow+\infty}{\longrightarrow} \chi^{2}(2) .
$$

In Jarque and Bera (1980), the constant is in the regressors. Hence, we have

$$
\sum_{t=1}^{T} \hat{u}_{t}=0
$$

In addition, $\hat{\alpha}^{2}$ is given by $\hat{\alpha}^{2}=T^{-1} \sum_{t=1}^{T}\left(y_{t}-x_{t}^{\top} \hat{\beta}\right)^{2}$. Therefore, we have

$$
\frac{1}{T} \sum_{t=1}^{T} \hat{u}_{t}^{2}=1
$$

Hence, when one combines the left-hand side of (4.20) with (4.21) and (4.22), one gets

$$
\frac{1}{6}\left(\frac{1}{\sqrt{T}} \sum_{t=1}^{T}\left[\hat{u}_{t}^{3}-\hat{u}_{t}\right]\right)^{2}+\frac{1}{24}\left(\frac{1}{\sqrt{T}} \sum_{t=1}^{T}\left[\hat{u}_{t}^{4}-6 \hat{u}_{t}^{2}+3\right]\right)^{2}, \text { i.e. }
$$




$$
\left(\frac{1}{\sqrt{T}} \sum_{t=1}^{T} H_{3}\left(\hat{u}_{t}\right)\right)^{2}+\left(\frac{1}{\sqrt{T}} \sum_{t=1}^{T} H_{4}\left(\hat{u}_{t}\right)\right)^{2}
$$

In other words, Jarque and Bera (1980) test coincides with the joint test based on third and fourth Hermite polynomials. However, the setting they considered is less general than ours. In addition, their estimation method is the ML while in our case we only need a square-root $T$ consistent estimator.

In summary, when one wants to test normality, $\mathcal{N}(0,1)$, through skewness and excess kurtosis, one has two methods that are robust against parameter uncertainty. One can either use the third and fourth Hermite polynomials on the fitted residuals whatever the estimation method, or, use the Jarque and Bera (1980) test on the standardized residuals, i.e., the fitted residuals minus their empirical mean divided by their standard deviation. ${ }^{10}$ Of course, for time series, the second method is not valid while one has to use a HAC method for estimating the variance-covariance matrix in the first method.

\section{A Monte Carlo study}

In this section, we provide some Monte Carlo experiments to study the finite sample properties of the tests we proposed. We also compare our tests with the most popular ones, that is the Kolmogorov-Smirnov and the Jarque-Bera tests. These two tests are respectively denoted by KS and JB in the tables. Note that when we consider the parameter uncertainty problem, we also provide the Lilliefors modified Kolmogorov-Smirnov test and denoted by M-KS in the tables.

All the test functions we consider are based on Hermite polynomials given their robustness against parameter uncertainty. We consider test functions based on individual Hermite polynomials $H_{i}$ for $i=3, \ldots, 10$. We also consider joint tests based on $\left(H_{3}, H_{4}, \ldots, H_{i}\right)$, for $i=4, \ldots, 10$. These tests are denominated by $H_{3-i}$ for $i=4, \ldots, 10$ in the tables.

In all the simulations experiments, we consider four sample sizes: 100, 250, 500 and 1000. All the results are based on 50000 replications. We report in the tables the empirical probability of rejecting the null hypothesis when one considers tests at $5 \%$ significance level. Tests based on $10 \%, 2.5 \%$ and $1 \%$ are similar to $5 \%$ and are omitted to save space. ${ }^{11}$

Cross-sectional case. We start by simulating an i.i.d. sample from a $\mathcal{N}(0,1)$. We assume that we know the mean and the variance. Obviously, this is unrealistic in practice. However, this is a good benchmark for the realistic cases where the parameters are unknown and have to be estimated. We reported the results in Table 1. Consider the tests based on individual

\footnotetext{
${ }^{10}$ These two methods are sufficient ones. In particular, Fiorentini, Sentana and Calzolari (2002) show that for some heteroskedastic models estimated by the ML method, test based on the fourth moment (as in Jarque and Bera, 1980) applied to the fitted residuals is still valid.

${ }^{11}$ They are available upon request from the authors.
} 
Hermite polynomials. Their finite sample properties are clearly good. In particular, they do not reject the null more than the nominal level, even with the smaller sample size. However, while the tests based on higher order polynomials $H_{i}$ for $i \geq 6$ underreject the null for the four sample sizes, this is not problematic given that we are considering the level of the tests. The tests based on several Hermite polynomials have also very good finite sample properties for the four sample sizes. Indeed, we do not observe the under rejection when we use high order Hermite polynomials.

Consider now the popular tests, i.e. KS and JB. The KS test works very well whatever the sample size. Interestingly, the properties of tests based on the Hermite polynomials are very close to the KS test and some times better for the sample size 100 when one considers a test based on $H_{4}$. However the JB test does not work well and overreject the null. The main reason is that when the empirical mean of the sample is not zero, the asymptotic distribution of the Jarque and Bera (1980) test is not a $\chi^{2}(2)$.

In Table 2, we present the results of the same tests ${ }^{12}$ on the same samples when one does not know the mean and the variance and estimates them. Thus, the test statistics are based on the standardized residuals. By comparing Table 2 with Table 1, it is clear that tests based on Hermite polynomials underreject a little bit the null assumption which again is not problematic. However the difference between knowing or not the mean and the variance decreases with the sample size and almost vanishes when the sample size is 1000. This confirms the robustness of these tests against parameter uncertainty. This is in contrast with the Kolmogorov-Smirnov test that almost never rejects the null. However, the Lilliefors modified test works well whatever the sample size. This is also the case for the JB test since by construction, the empirical mean of the standardized residuals is zero. Indeed, the JB test coincides with the joint test based on $H_{3}$ and $H_{4}$, that is $H_{3-4}{ }^{13}$

We now study the power of the considered tests against some interesting alternative assumptions for the cross-sectional case. In particular, we consider Student, chi-square and exponential alternatives. We start by simulating i.i.d. random variables from Student distributions with six different degrees of freedom: a) $T(60)$; b) $T(30)$; c) $T(20)$; d) $T(10)$; e) $T(6)$; f) $T(3)$. Recall that for a random variable that follows a $T(\nu)$ distribution, the moments of order higher than $\nu-1$ are not defined. Hence, the moments of $H_{i}$ are not defined if $i>\nu-1$. Moreover, the asymptotic distribution of the corresponding test statistics are not chi-square if $2 i>\nu-1$ since the variance of the Hermite polynomial $H_{i}$ is not defined. The results are presented in Table 3. It is clear that the power of the tests is low when the degree of freedom $\nu$ is high. This is not surprising since a $T(\nu)$ distribution tends toward a normal one when $\nu \rightarrow+\infty$. However, when the degree of freedom decreases, the power of the tests increases

\footnotetext{
${ }^{12}$ We do not consider $H_{1}$ and $H_{2}$ since these moments are used to estimate the mean and the variance.

${ }^{13}$ There is a small difference in Table 2 between JB and $H_{3-4}$ tests since in JB test, the variance is estimated by $T^{-1} \sum_{t=1}^{T}\left(x_{t}-\bar{X}\right)^{2}$ while in the Hermite case it is estimated by $(T-1)^{-1} \sum_{t=1}^{T}\left(x_{t}-\bar{X}\right)^{2}$ where $T$ is the sample size and $\bar{X}$ the empirical mean.
} 
and becomes very good when the degree of freedom is smaller than 10 , which is the relevant case in the volatility literature; see the first example in the empirical section. A surprising result is that the fourth Hermite polynomial captures much more the non normality than the higher polynomials. In contrast, tests based on odd polynomials do not work well. This is not surprising given that the mean in population of any odd Hermite polynomial is zero (when it is well defined) for any symmetric distribution and, hence, for a Student one.

In order to understand the behavior of the power of test statistics against Student distributions, we characterize in the following proposition their behavior for the third and fourth Hermite polynomials.

Proposition 5.1 Let $y_{1}, y_{2}, \ldots, y_{T}$, an i.i.d. sample of a random variable $Y$ that follows a $T(\nu)$ where $\nu$ is assumed to be higher than eight $(\nu>8)$, and define the random variable $X$ by $X=Y \sqrt{(\nu-2) \nu^{-1}}$. Then:

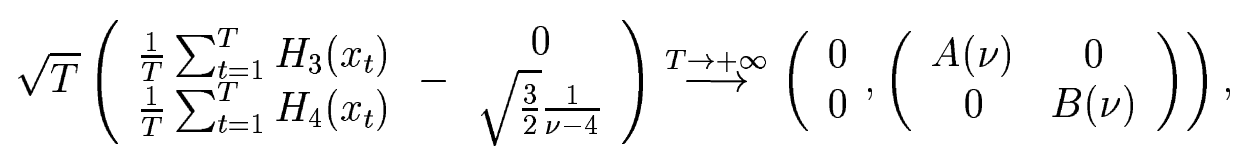

where

$$
A(\nu)=\frac{\nu^{2}-\nu+10}{(\nu-6)(\nu-4)} \text { and } B(\nu)=\frac{24 \nu^{3}+1321 \nu^{2}+708 \nu-1572}{(\nu-8)(\nu-6)(\nu-4)}
$$

As a consequence:

$$
\begin{gathered}
\left(\frac{1}{\sqrt{T}} \sum_{t=1}^{T} H_{3}\left(x_{t}\right)\right)^{2} \stackrel{T \rightarrow+\infty}{\longrightarrow} A(\nu) \chi^{2}(1), \text { and } \\
\left(\frac{1}{\sqrt{T}} \sum_{t=1}^{T} H_{4}\left(x_{t}\right)\right)^{2} \stackrel{T \rightarrow+\infty}{\longrightarrow}+\infty
\end{gathered}
$$

In addition, when $T$ is large, we have the following approximation result:

$$
\left(\frac{1}{\sqrt{T}} \sum_{t=1}^{T} H_{4}\left(x_{t}\right)\right)^{2} \sim \frac{1}{T} B(\nu) \chi^{2}\left(1, T^{2} C(\nu)\right) \text { where } C(\nu)=\frac{3}{2} \frac{1}{(\nu-4) B(\nu)} .
$$

Equation (5.3) implies that when one uses the third Hermite polynomial for testing normality while the random variable is a Student $T(\nu)$ at, say, $5 \%$ level, one accepts normality with a probability that equals $P\left(A(\nu) \chi^{2}(1)>3.84\right)$ if one assumes that

$$
\left(\frac{1}{\sqrt{T}} \sum_{t=1}^{T} H_{3}\left(x_{t}\right)\right)^{2} \stackrel{T \rightarrow+\infty}{\longrightarrow} \chi^{2}(1) .
$$

In Table 4, we provided for all values of $\nu$ we considered in the Monte Carlo experiment, the value of $A(\nu)$ and the probability $P\left(A(\nu) \chi^{2}(1)>3.84\right)$. These results are compatible with the 
Monte Carlo ones; in particular, the theoretical probabilities of rejection are very close to the Monte Carlo ones for the sample size $T=1000$. Given that a test based on the third Hermite polynomial is not powerful, this is also the case for any test that uses this polynomial, as the joint test based on the third and fourth polynomial. In contrast, equation (5.4) explains why a test based on the fourth Hermite polynomial has a good asymptotic power against Student distribution.

Consider now the power of the tests against a $\chi^{2}(1)$ and an exponential distribution, $\exp (1)$. The results are reported in Table 5. They clearly imply that tests based on the third and fourth Hermite polynomials are very powerful whatever the sample size and that they are similar to the modified Kolmogorov-Smirnov test. However, tests based on individual higher order Hermite polynomials are less powerful for small sample sizes.

Dependent case. Consider now the dependent case, where the variable of interest is serially correlated. We consider several autoregressive normal processes of order one, AR(1), i.e., we assume that the conditional distribution of the variable of interest denoted by $x_{t}$ given its past is $\mathcal{N}\left(\rho x_{t-1}, 1-\rho^{2}\right)$. Observe that the marginal distribution of $x_{t}$ is $\mathcal{N}(0,1)$. We consider four values for $\rho$ : a) $\rho=.1$; b) $\rho=.5$; c) $\rho=.7$ and d) $\rho=0.9$. We did the same tests as for the independent case by assuming that do not know the unconditional mean and variance of $x_{t}$.

We start by ignoring the dependence of the data, that is we assume that the sample size is i.i.d.; the results are reported in Table 6a. They clearly mean that all the tests, including M-KS and JB ones, overreject the null when the sample size is higher than 250. This distortion is problematic. Therefore, we take into account the dependence of the data.

Next, we assume that we know the autoregressive structure. This is not always a realistic assumption. We do it however in order to get a benchmark. We consider two cases; in the first one, we assume that we know the autoregressive parameter while we estimate it by OLS in the second case. Given that the autoregressive feature of the data is known, we assume that the weighting matrix that appears in the test statistic is diagonal and that the diagonal coefficients are given by (3.11). The results are provided in Table $6 \mathrm{~b}$ and Table $6 \mathrm{c}$. These results are clearly good and similar to the ones provided in Table 2 for the independent case. We observe again an under rejection of normality, in particular when the autoregressive coefficient increases.

We then test normality by ignoring the autoregressive feature of the data but by taking into account their dependence. Therefore, we do not assume that the weighting matrix $\Sigma$ is diagonal. Instead, we estimate it by a HAC method. The HAC method is developed by using the quadratic kernel with an automatic lag selection procedure of Andrews (1991). The results are reported in Table 6d. From this table, it is clear that univariate tests work well. However, joint tests overreject the normality assumption, especially for small sample sizes and for tests that are based on three or more Hermite polynomials. The overrejection is relatively small for the test based on third and fourth Hermite polynomials. 
We now study the power of these tests against an autoregressive model of order one where the innovation is a Student one. Again, with the same autoregressive parameters as previously, we consider the same degree of freedom as in the i.i.d. case, i.e., 60, 30, 20, 10, 6 and 3. Observe that the marginal distribution of the processes are (probably) not Student. However, their tails are clearly fatter than for a normal distribution. The results are reported in Tables $7 \mathrm{a}, 7 \mathrm{~b}, 7 \mathrm{c}$, $7 \mathrm{~d}, 7 \mathrm{e}$ and $7 \mathrm{f}$. We also report in Table $7 \mathrm{~g}$ the results of the example where the innovation is $T(5)$. We consider this example for comparison purposes with Bai and $\mathrm{Ng}$ (2001). The main results of the tables can be summarized as follows. The test based on univariate polynomials and different from the third one work well; however, their power decreases when both the degree of freedom of the Student distribution and the autocorrelation parameter are high. The tests univariate, bivariate and trivariate tests based on the third Hermite polynomials (denoted in the tables by $H_{3}, H_{3-4}$ and $H_{3-5}$ ) are not powerful, especially when the autocorrelation is high. The main reason is symmetry. The second reason, given by Bai and Ng (2001), is that when the autocorrelation parameter is high, the Central Limit Theorem suggests that process of interest is close to a normal one. Note however that our results for $\mathrm{H}_{3-4}$ are different from ones of Bai and $\mathrm{Ng}$ (2001) when they test normality (for $\nu=5$ ) since that they find that their test are powerful. The main reason is that we do not assume diagonality of the weighting matrix when we do joint tests while Bai and $\mathrm{Ng}$ (2001) do. As we pointed out in the third section, there is no reason to assume in general that the tests are asymptotically independent. However, it is the case for an $\mathrm{AR}(1)$ process, i.e., the setup we adopted as well as Bai and $\mathrm{Ng}$ (2001) in our simulation. Given that we are interested on the power of the tests, we did not take into account this asymptotic independence in our simulations.

\section{Empirical examples}

In this section we provide three empirical examples. The first one concerns GARCH models while the second and third ones deal with high frequency realized volatility.

\subsection{First example: GARCH model}

A very popular model in the volatility literature is $\operatorname{GARCH}(1,1)$ of Bollerslev (1986). More precisely, Bollerslev (1986) generalizes the ARCH models of Engle (1982) by assuming that

$$
y_{t}=\sqrt{h_{t}} u_{t} \text { with } h_{t}=\omega+\alpha y_{t-1}^{2}+\beta h_{t-1} \text {, where } \omega \geq 0, \alpha \geq 0, \beta \geq 0, \alpha+\beta<1,
$$

and the process $u_{t}$ is assumed to be i.i.d. and $\mathcal{N}(0,1)$. An important characteristic of GARCH models is that the kurtosis of $y_{t}$ is higher than for a normal variable. It turns out that financial returns are also leptokurtic and, hence, GARCH models describe well financial data. ${ }^{14}$

\footnotetext{
${ }^{14}$ The second characteristic that GARCH models share with financial returns is the clustering effect. For a survey on GARCH models, see for instance Bollerslev, Engle and Nelson (1994).
} 
However, some empirical studies showed that the implied kurtosis of a $\operatorname{GARCH}(1,1)$ is lower than empirical ones. These studies lead Bollerslev (1987) to assume that the standardized process $u_{t}$ may follow a Student distribution. Under this assumption, $\operatorname{GARCH}(1,1)$ fit very well financial returns. Indeed, by using Bayesian likelihood method, Kim, Shephard and Chib (1998) showed that a Student GARCH(1,1) outperforms in terms of likelihood another very popular volatility model, namely the log-normal stochastic volatility model of Taylor (1986) popularized by Harvey, Ruiz and Shephard (1994).

The first example we consider in our empirical study is testing normality of the standardized residual $u_{t}$. We consider the same data as Harvey, Ruiz and Shephard (1994) and Kim, Shephard and Chib (1998), ${ }^{15}$ i.e., observations of weekday close exchange rates from 1/10/81 to $28 / 6 / 85$. The exchange rates are the U.K. Pound, French Franc, Swiss Franc and Japanese Yen, all against the U.S. Dollar. We estimate the model by a Gaussian QML method. The method is consistent as soon as the variance $h_{t}$ is well specified (Bollerslev and Wooldridge, 1992). We get the fitted residuals $\hat{u}_{t}$ and test their normality. The results are provided in Table 8. It is clear that normality of the residuals is strongly rejected by all the tests, in particular those related to the tails (even polynomials). The difference between JB and $H_{3-4}$ tests is relatively small; this fact is in line with the results of Fiorentini, Sentana and Calzolari (2002) who showed that the test based on the fourth moment for GARCH models is still valid even if the parameters are estimated..$^{16}$ Observe that the magnitude of normality rejection is in the following increasing order: FF-US\$, UK-US\$, SF-US\$, and Yen-US\$. Interestingly, this order is the same than one implied by the Student GARCH models estimated by Kim, Shephard and Chib (1998), since these authors reported in their Table 13 the following degree of freedom: $12.82,9.71,7.57$ and 6.86 .

\subsection{Second and third examples: realized volatility}

Several recent studies highlight the advantage of using high-frequency data to measure volatility of financial returns. These include Andersen and Bollerslev (1998), ABDL (2001) and Barndorff-Nielsen and Shephard (2001); for a survey of this literature, Andersen, Bollerslev and Diebold (2001) and Barndorff-Nielsen and Shephard (2002) should be consulted. Typically, when one is interested in volatility over, say, a day, then these papers propose to study the estimation of this volatility by the sum of the intra-daily squared returns, such as returns over five or thirty minutes. This measure of volatility is called the realized volatility.

More precisely, consider $S_{t}$ a continuous time process representing the price of an asset or the exchange rate between two currencies. Assume that it is characterized by the following

\footnotetext{
${ }^{15}$ We are grateful to Neil Shephard for providing us with the data.

${ }^{16}$ Recall that exchange rates returns are symmetric.
} 
stochastic differential equation:

$$
d \log \left(S_{t}\right)=m_{t} d t+\sigma_{t} d W_{t} \text { with } d \sigma_{t}^{2}=\tilde{m}_{t} d t+\tilde{\sigma}_{t} d \tilde{W}_{t}
$$

where $W_{t}$ and $\tilde{W}_{t}$ are standard Brownian processes, potentially correlated. Assume that the time $t$ is measured in units of one day. Consider a real $h$ such that $1 / h$ is a positive integer. Then, integrated and realized volatility denoted respectively by $I V_{t}$ and $R V_{t}(h)$ are defined by

$$
I V_{t} \equiv \int_{t-1}^{t} \sigma_{u}^{2} d u \quad \text { and } \quad R V_{t}(h) \equiv \sum_{i=1}^{1 / h} r_{t-1+i h}^{(h) 2}
$$

where $r_{t-1+i h}^{(h)}$ is the return over the period $[t-1+(i-1) h ; t-1+i h]$, given by $r_{t-1+i h}^{(h)} \equiv$ $\log \left(S_{t-1+i h}\right)-\log \left(S_{t-1+(i-1) h}\right)$. It turns out that when $h$ goes to zero, realized volatility $R V_{t}(h)$ converges (in probability) to integrated volatility $I V_{t}$.

\subsubsection{Second example: distribution of standardized residuals}

In their forecasting paper, ABDL (2002) presented empirical results suggesting that the standardized residuals $\varepsilon_{t}(h)$ defined by $\varepsilon_{t}(h) \equiv\left(r_{t} / \sqrt{R V_{t}(h)}\right)$ where $r_{t}$ is the daily return, i.e. $r_{t}=\log \left(S_{t}\right)-\log \left(S_{t-1}\right)$, are $\mathcal{N}(0,1)$. These empirical results were based on the skewness, kurtosis, and nonparametric estimation of density of $\varepsilon_{t}(h)$. This is our second example.

We consider the same data as in ABDL (2002), ${ }^{17}$ i.e. returns of three exchange rates, DM-US\$, Yen-US\$ and Yen-DM, from December 1, 1986 through June 30, 1999. The realized volatilities are based on observations at five and thirty minutes. Therefore, we have six series. For each variable, we define the standardized residuals and test their normality.

We start by testing that the standardized residuals are $\mathcal{N}(0,1)$, i.e., we assume as $\operatorname{ABDL}(2002)$ that the mean and variance of the normal distribution are known and equal to zero and one respectively. The results are provided in Table 9a. In Panel A, we provide the results when one assumes that the standardized residuals are independent. Given that this independence is not obvious, we also provided in Panel B results of the test statistics that do not assume the independence and use a HAC method for estimating the weighting matrix.

The results form Table 9a clearly reject the $\mathcal{N}(0,1)$ assumption, particularly test based on the fourth Hermite polynomial which is powerful. Note that the results in Panel B are close but different from ones of Panel B, suggesting that some feature (like dependence or heteroskedasticity) of the data are not taken into account in Panel A.

Another striking result in Panel A is the difference between the Jarque-Bera (JB) test and the test based on third and fourth Hermite polynomials $\left(H_{3-4}\right)$. This difference suggests that the mean of the residuals is not zero or that their variance is not one. Therefore, we also

\footnotetext{
${ }^{17}$ We are grateful to Ramazan Gençay for providing us the OLSEN data and to Torben Andersen and Paul Labys for providing us their data.
} 
test normality of the residuals with unknown mean and variance. The results are reported in Panel A of Table 9b when one assumes independence and in Panel B when one estimates the weighting matrix by a HAC procedure. The main message from Table $9 \mathrm{~b}$ is that normality is not rejected for standardized residuals based on five-minute realized volatility and rejected for ones based on thirty-minute realized volatility.

\subsubsection{Third example: distribution of realized volatility}

Another assumption made in ABDL (2002) is conditional normality of the log of realized volatility. Hence, log-realized volatility are also unconditionally normal. This is our third example. In Table 10, we provide the results of testing normality of log-realized volatility with unknown mean and variance. The weighting matrix is again estimated by a HAC procedure of Andrews (1991). It is clear that unconditional normality of log-realized volatility is rejected, particularly for realized volatility based on five-minute returns. ${ }^{18}$ Observe that the rejection is due to the asymmetry of the distribution. In this test, we assume that the weighting matrix is well defined. This is not necessarily the case. In particular, ABDL (2002) reported results that clearly indicate a presence of long memory in log-realized volatility. In this case, the weighting matrix is not well defined and our test procedures are not valid. However, this is also the case for the procedures of ABDL (2002). which are based on the skewness, kurtosis, and nonparametric estimation of density of log-realized volatilities. Testing normality under long memory is more difficult and is left for future research.

\section{Conclusion}

In this paper, we consider testing marginal normal distributional assumptions for both crosssection and time series data. We use the GMM approach to test moment conditions given by Stein (1972) equations and the first class of moment conditions derived by Hansen and Scheinkman (1995) when the process of interest is a scalar diffusion. The main advantage of our approach is that tests based on Hermite polynomials are robust against parameter uncertainty. In addition, the GMM setting is well suited to take into account serial correlation by using a HAC procedure. We provide simulation results that clearly show the usefulness of our approach. We also apply our approach to test for normality in three volatility models.

Three main extensions have to be considered. The first one is to extend our approach to the multivariate case. The second one is to consider other distributions, in particular Pearson ones. These two extensions are under consideration by using the Hansen and Scheinkman (1995) moment conditions which are valid in both multivariate normal and non normal cases. A third important extension will be testing normality under long memory.

\footnotetext{
${ }^{18}$ In their study, ABDL (2002) used thirty-minute realized volatilities.
} 
Ait-Sahalia, Y. (1996), "Testing Continuous-Time Models of the Spot Interest Rate," Review of Financial Studies, 9, 385-426.

Ait-Sahalia, Y., L.P. Hansen and J. Scheinkman (2001), "Operator Methods for Continuous-Time Markov Models," in Y. Ait-Sahalia and L.P. Hansen (Eds.), Handbook of Financial Econometrics, forthcoming.

Andersen, T.G. and T. Bollerslev (1998), "Answering the Skeptics: Yes, Standard Volatility Models Do Provide Accurate Forecasts," International Economic Review, $39,885-905$.

Andersen, T.G., T. Bollerslev and F.X. Diebold (2001), "Parametric and Nonparametric Measurements of Volatility," in Y. Ait-Sahalia and L.P. Hansen (Eds.), Handbook of Financial Econometrics, forthcoming.

Andersen, T.G., T. Bollerslev, F.X. Diebold and P. Labys (2001), "The Distribution of Exchange Rate Volatility," Journal of the American Statistical Association, 96, 42-55.

Andersen, T.G., T. Bollerslev, F.X. Diebold and P. Labys (2002), "Modeling and Forecasting Realized Volatility," unpublished manuscript, Northwestern University.

Andersen, T.G., H.J. Chung and B.E. Sorensen (1999), "Efficient Method of Moment Estimation of a Stochastic Volatility Model: A Monte Carlo Study," Journal of Econometrics, 91, 61-87.

Andrews, D.W.K. (1991), "Heteroskedasticity and Autocorrelation Consistent Covariance Matrix Estimation", Econometrica, 60, 953-966.

Bai, J. and S. Ng (2001), "Tests for Skewness, Kurtosis, and Normality for Time Series Data," unpublished manuscript, Boston College.

Barndorff-Nielsen, O.E. and N. Shephard (2001), "Non-Gaussian OU based Models and some of their uses in Financial Economics," Journal of the Royal Statistical Society, $B, 63,167-241$.

Barndorff-Nielsen, O.E. and N. Shephard (2002), " Estimating Quadratic Variation Using Realised Variance," Journal of Applied Econometrics, forthcoming.

Bera, A.K. and C.M. Jarque (1981), "Efficient Tests for Normality, Homoscedasticity and serial Independence of Regression Residuals: Monte Carlo Evidence," Economics Letters, 7, 313-318.

Bera, A. and S. John (1983), "Tests for Multivariate Normality with Pearson Alternatives," Commun. Statist. Theor. Meth., 12, 103-107.

Berkowitz, J. (2001), "Testing Density Forecasts, With Applications to Risk Management," Journal of Business \& Economic Statistics, 19, 465-474.

Bollerslev, T. (1986), "Generalized Autoregressive Conditional Heteroskedasticity," Journal of Econometrics, 31, 307-327.

Bollerslev, T. (1987), “ A Conditional Heteroskedastic Time Series Model For Speculative Prices and Rates of Returns," Review of Economics and Statistics, 69, 542-547.

Bollerslev, T., R. Engle and D.B. Nelson (1994), "ARCH Models," Handbook of Econometrics, Vol IV. 
Bollerslev, T. et J.F. Wooldridge (1992), "Quasi Maximum Likelihood Estimation and Inference in Dynamic Models with Time Varying Covariances", Econometric Reviews, $11,143-172$.

Bowman, K.O. and L.R. Shenton (1975), "Omnibus Test Contours for Departures from Normality based on $\sqrt{b}_{1}$ and $b_{2}$, " Biometrika, 62, 243-250.

Chen, X., L.P. Hansen and J. Scheinkman (2000), "Principal Components and the Long Run," unpublished manuscript, University of Chicago.

Christoffersen, P.F. (1998), "Evaluating Interval Forecasts," International Economic Review, 39, 841-862.

Conley, T., L.P. Hansen, E. Luttmer and J. Scheinkman (1997), "Short-Term Interest Rates as Subordinated Diffusions," Review of Financial Studies, 10, 525-577.

Davidson, R. and J.G. MacKinnon (1993), "Estimation and Inference in Econometrics," Oxford University Press.

Diebold, F.X., T.A. Gunther and A.S. Tay (1998), "Evaluating Density Forecasts, with Applications to Financial Risk Management," International Economic Review, 39, 863-883.

Dufour, J-M, A. Farhat, L. Gardiol and L. Khalaf (1998), "Simulation-Based Finite Sample Normality Tests in Linear Regressions," Econometrics Journal, 1, C154-C173.

Engle, R.F. (1982), "Autoregressive Conditional Heteroskedasticity with Estimates of the Variance of United Kingdom Inflation," Econometrica, 50, 987-1007.

Epps, T.W and L.B. Pulley (1983), "A Test for Normality Based on the Empirical Characteristic Function," Biometrika, 70, 723-726.

Epps, T.W, K. Singleton and L.B. Pulley (1982), "A Test of Separate Families of Distributions Based on the Empirical Moment Generating Function," Biometrika, 69, 391-399.

Fiorentini, G., E. Sentana and G. Calzolari (2002), "The Score of Conditionally Heteroskedastic Dynamic Regression Models with Student t Innovations, an LM Test for Multivariate Normality," unpublished manuscript, CEMFI.

Gallant, A.R. and G. Tauchen (1999), "The Relative Efficiency of Methods of Moments Estimators," Journal of Econometrics, 92, 149-172.

Gouriéroux, C. and A. Monfort (1996), Simulation-Based Econometric Methods, CORE Lectures, Oxford.

Hansen, L.P. (1982), "Large Sample Properties of Generalized Method of Moments Estimators," Econometrica, 50, 1029-1054.

Hansen, L.P. and J. Scheinkman (1995), "Back to the Future: Generating Moment Implications for Continuous Time Markov Processes," Econometrica, 63, 767-804.

Harvey, A.C., E. Ruiz and N. Shephard (1994), "Multivariate Stochastic Variance Models," Review of Economic Studies, 61, 247-264.

Jacquier, E., N.G. Polson and P.E. Rossi (1994), "Bayesian Analysis of Stochastic Volatility Models," Journal of Business and Economic Statistics, 12, 371-389.

Jarque, C.M. and A.K. Bera (1980), "Efficient Tests for Normality, Homoscedasticity and serial Independence of Regression Residuals," Economics Letters, 6, 255-259. 
Kim, S., N. Shephard and S. Chib (1998), "Stochastic Volatility: Likelihood Inference and Comparison with ARCH Models," Review of Economic Studies, 65, 361-393.

Kiefer, N.M. and M. Salmon (1983), "Testing Normality in Econometric Models," Economics Letters, 11, 123-127.

Kilian, L. and U. Demiroglu (2000), "Residual-Based Test for Normality in Autoregressions: Asymptotic Theory and Simulation Evidence," Journal of Business E Economic Statisitcs, 18, 40-50.

Kolmogorov, A.N. (1933), "Sulla Determinazione Empiricadi una Legge di Distribuzione," Giorna. Ist. Attuari., 4, 83-91.

Koutrouvelis, I.A. (1980), "A Goodness-of-Fit Test of Simple Hypotheses Based on the Empirical Characteristic Function," Biometrika, 67, 238-240.

Koutrouvelis, I.A. and J. Kellermeier (1981), "A Goodness-of-Fit Test Based on the Empirical Characteristic Function when Parameters must be Estimated," $J . R$. Statist. Soc. B, 43, 173-176.

Lilliefors, H.W. (1967), "On the Kolmogorov-Smirnov Test for Normality with Mean and Variance," Journal of the American Statistical Association, 62, 399-402.

Mardia, K.V. (1970), "Measures of Multivariate Skewness and Kurtosis with Applications," Biometrika, 57, 519-530.

Nelson, D.B. (1991), "Conditional Heteroskedasticity in Asset Returns: A New Approach," Econometrica, 59, 347-370.

Newey W.K. (1985), "Generalized Method of Moments Specification Testing," Journal of Econometrics, 29, 229-256.

Newey W.K. and K.D. West (1987), "A Simple, Positive Semi-Definite, Heteroskedasticity and Autocorrelation Consistent Covariance Matrix," Econometrica, 55, 703-708.

Richardson, M. and T. Smith (1993), "A test for Multivariate Normality in Stock Returns," Journal of Business, 66, 295-321.

Schoutens, W. (2000), Stochastic Processes and Orthogonal Polynomials, Lecture Notes in Statistics 146, Springer-Verlag.

Smirnov, N.V. (1939), "Sur les Écarts de la Courbe de Distribution Empirique," French/Russian Su Matematiceskii Sbornik N. S., 6, 3-26.

Stein, C. (1972), "A Bound for the Error in the Normal Approximation to the Distribution of a Sum of Dependant Random Variables," Proc. Sixth Berkeley Symp. Math. Statist. Probab., 2, 583-602.

Tauchen, G.E. (1985), "Diagnostic Testing and Evaluation of Maximum Likelihood Models," Journal of Econometrics, 30, 415-443.

Taylor, S.J. (1986), Modeling Financial Time Series, John Wiley.

van der Klaauw, B. and R.H. Koning (2001), "Testing the Normality Assumption in the Sample Selection Model with an Application to Travel Demand," Journal of Business \& Economic Statisitcs, forthcoming.

White, H. (1982), "Maximum Likelihood Estimation of Misspecified Models," Econometrica, 50, 1-25.

White, H. and G.M. MacDonald (1980), "Some Large-Sample Tests for Nonnormality in the Linear Regression Model," Journal of the American Statistical Association, 75, $16-28$. 
Table 1: Size of the tests. Mean and variance are known.

\begin{tabular}{lcccc}
\hline \multicolumn{1}{c}{$\mathrm{T}$} & 100 & 250 & 500 & 1000 \\
$H_{1}$ & .0508 & .0514 & .0525 & .0500 \\
$H_{2}$ & .0511 & .0485 & .0498 & .0486 \\
$H_{3}$ & .0549 & .0542 & .0556 & .0515 \\
$H_{4}$ & .0475 & .0470 & .0494 & .0498 \\
$H_{5}$ & .0362 & .0431 & .0483 & .0504 \\
$H_{6}$ & .0205 & .0266 & .0320 & .0361 \\
$H_{7}$ & .0128 & .0156 & .0199 & .0239 \\
$H_{8}$ & .0135 & .0116 & .0126 & .0142 \\
$H_{9}$ & .0188 & .0168 & .0143 & .0128 \\
$H_{10}$ & .0122 & .0160 & .0184 & .0158 \\
$H_{3-4}$ & .0582 & .0562 & .0565 & .0531 \\
$H_{3-5}$ & .0599 & .0624 & .0632 & .0599 \\
$H_{3-6}$ & .0544 & .0580 & .0609 & .0611 \\
$H_{3-7}$ & .0503 & .0525 & .0552 & .0558 \\
$H_{3-8}$ & .0504 & .0499 & .0513 & .0512 \\
$H_{3-9}$ & .0480 & .0488 & .0500 & .0487 \\
$H_{3-10}$ & .0445 & .0458 & .0484 & .0474 \\
$\mathrm{KS}$ & .0446 & .0470 & .0493 & .0486 \\
$\mathrm{JB}$ & .1498 & .1632 & .1743 & .1741 \\
\hline \hline
\end{tabular}

Note: The data are i.i.d. from a $\mathcal{N}(0,1)$ distribution.

We test the $\mathcal{N}(0,1)$ assumption. Thus, we do not estimate the mean and standard deviation. The

results are based on 50000 replications. For each sample size, we provide the frequency of rejection at a $5 \%$ level. $H_{i-j}$ corresponds to the joint test based on $H_{k}, i \leq k \leq j$. KS and JB are the

Kolmogorov-Smirnov and Jarque-Bera tests. 
Table 2: Size of the tests.

Mean and variance are estimated.

\begin{tabular}{|c|c|c|c|c|}
\hline$\overline{\mathrm{T}}$ & 100 & 250 & 500 & 1000 \\
\hline$H_{3}$ & .0429 & .0475 & .0490 & .0497 \\
\hline$H_{4}$ & .0308 & .0378 & .0427 & .0456 \\
\hline$H_{5}$ & .0242 & .0360 & .0432 & .0478 \\
\hline$H_{6}$ & .0121 & .0214 & .0288 & .0342 \\
\hline$H_{7}$ & .0068 & .0122 & .0168 & .0217 \\
\hline$H_{8}$ & .0077 & .0086 & .0101 & .0130 \\
\hline$H_{9}$ & .0123 & .0143 & .0112 & .0105 \\
\hline$H_{10}$ & .0060 & .0126 & .0166 & .0151 \\
\hline$H_{3-4}$ & .0410 & .0455 & .0463 & .0480 \\
\hline$H_{3-5}$ & .0418 & .0513 & .0532 & .0543 \\
\hline$H_{3-6}$ & .0361 & .0473 & .0526 & .0553 \\
\hline$H_{3-7}$ & .0331 & .0423 & .0469 & .0506 \\
\hline$H_{3-8}$ & .0325 & .0396 & .0429 & .0464 \\
\hline$H_{3-9}$ & .0309 & .0389 & .0422 & .0440 \\
\hline$H_{3-10}$ & .0284 & .0368 & .0406 & .0428 \\
\hline $\mathrm{KS}^{1}$ & .0001 & .0002 & .0001 & .0002 \\
\hline $\mathrm{M}-\mathrm{KS}^{2}$ & .0519 & .0537 & .0567 & .0596 \\
\hline $\mathrm{JB}^{3}$ & .0433 & .0473 & .0470 & .0485 \\
\hline
\end{tabular}

Note: The data are i.i.d. from a $\mathcal{N}(0,1)$ distribution.

We test the normality assumption. Thus, we

estimate the mean and standard deviation. The

results are based on 50000 replications. For each

sample size, we provide the frequency of rejection at

a $5 \%$ level. $H_{i-j}$ corresponds to the joint test based

on $H_{k}, i \leq k \leq j$. KS and JB are the

Kolmogorov-Smirnov and Jarque-Bera tests.

$\mathrm{M}-\mathrm{KS}$ is the modified Kolmogorov-Smirnov test. 
Table 3: Power of the tests against Student distributions.

\begin{tabular}{|c|c|c|c|c|c|c|c|c|c|}
\hline \multicolumn{5}{|c|}{ Panel A: $\nu=60$} & \multicolumn{5}{|c|}{ Panel B: $\nu=30$} \\
\hline $\mathrm{T}$ & 100 & 250 & 500 & 1000 & $\mathrm{~T}$ & 100 & 250 & 500 & 1000 \\
\hline$H_{3}$ & .0566 & .0649 & .0683 & .0680 & $H_{3}$ & .0744 & .0863 & .0897 & .0942 \\
\hline$H_{4}$ & .0487 & .0729 & .0949 & .1303 & $H_{4}$ & .0782 & .1330 & .1942 & .3033 \\
\hline$H_{5}$ & .0373 & .0614 & .0783 & .0946 & $H_{5}$ & .0559 & .0982 & .1294 & .1635 \\
\hline$H_{6}$ & .0210 & .0392 & .0546 & .0737 & $H_{6}$ & .0331 & .0660 & .0986 & .1373 \\
\hline$H_{7}$ & .0114 & .0236 & .0351 & .0539 & $H_{7}$ & .0202 & .0432 & .0710 & .1055 \\
\hline$H_{8}$ & .0122 & .0167 & .0243 & .0350 & $H_{8}$ & .0188 & .0300 & .0500 & .0715 \\
\hline$H_{9}$ & .0204 & .0245 & .0250 & .0276 & $H_{9}$ & .0325 & .0415 & .0445 & .0562 \\
\hline$H_{10}$ & .0124 & .0259 & .0347 & .0353 & $H_{10}$ & .0214 & .0479 & .0671 & .0737 \\
\hline$H_{3-4}$ & .0627 & .0836 & .1003 & .1255 & $H_{3-4}$ & .0930 & .1403 & .1915 & .2797 \\
\hline$H_{3-5}$ & .0636 & .0913 & .1129 & .1403 & $H_{3-5}$ & .0940 & .1488 & .2046 & .2937 \\
\hline$H_{3-6}$ & .0565 & .0855 & .1081 & .1387 & $H_{3-6}$ & .0859 & .1415 & .1974 & .2870 \\
\hline$H_{3-7}$ & .0515 & .0770 & .0994 & .1296 & $H_{3-7}$ & .0794 & .1302 & .1827 & .2701 \\
\hline$H_{3-8}$ & .0508 & .0740 & .0935 & .1211 & $H_{3-8}$ & .0774 & .1239 & .1734 & .2539 \\
\hline$H_{3-9}$ & .0486 & .0722 & .0913 & .1161 & $H_{3-9}$ & .0743 & .1222 & .1696 & .2448 \\
\hline$H_{3-10}$ & .0454 & .0682 & .0881 & .1131 & $H_{3-10}$ & .0696 & .1165 & .1637 & .2358 \\
\hline $\mathrm{KS}$ & .0001 & .0001 & .0001 & .0003 & $\mathrm{KS}$ & .0001 & .0002 & .0003 & .0004 \\
\hline M-KS & .0532 & .0602 & .0641 & .0689 & $-\mathrm{KS}$ & .0613 & .0687 & .0805 & .1003 \\
\hline JB & .0658 & .0854 & .1015 & .1263 & & .0973 & .1426 & .1932 & .2809 \\
\hline \multicolumn{5}{|c|}{ Panel C: $\nu=20$} & \multicolumn{5}{|c|}{ Panel D: $\nu=10$} \\
\hline $\mathrm{T}$ & 100 & 250 & 500 & 1000 & $\mathrm{~T}$ & 100 & 250 & 500 & 1000 \\
\hline$H_{3}$ & .0961 & .1172 & .1231 & .1296 & $H_{3}$ & .2508 & .3078 & .3345 & .3599 \\
\hline$H_{4}$ & .1117 & .2109 & .3423 & .5396 & $H_{4}$ & .3224 & .5921 & .8272 & .9722 \\
\hline$H_{5}$ & .0771 & .1420 & .1980 & .2526 & $H_{5}$ & .2058 & .3671 & .4945 & .6014 \\
\hline$H_{6}$ & .0489 & .1031 & .1579 & .2271 & $H_{6}$ & .1591 & .3158 & .4629 & .6181 \\
\hline$H_{7}$ & .0297 & .0719 & .1210 & .1862 & $H_{7}$ & .1094 & .2368 & .3780 & .5452 \\
\hline$H_{8}$ & .0270 & .0538 & .0858 & .1369 & $H_{8}$ & .1053 & .2056 & .3230 & .4793 \\
\hline$H_{9}$ & .0470 & .0670 & .0795 & .1104 & $H_{9}$ & .1450 & .2353 & .3079 & .4237 \\
\hline$H_{10}$ & .0340 & .0773 & .1113 & .1378 & $H_{10}$ & .1143 & .2486 & .3700 & .5021 \\
\hline$H_{3-4}$ & .1290 & .2148 & .3265 & .5001 & $H_{3-4}$ & .3374 & .5793 & .7997 & .9602 \\
\hline$H_{3-5}$ & .1285 & .2229 & .3348 & .4996 & $H_{3-5}$ & .3219 & .5666 & .7858 & .9526 \\
\hline$H_{3-6}$ & .1189 & .2126 & .3232 & .4876 & $H_{3-6}$ & .3086 & .5567 & .7811 & .9527 \\
\hline$H_{3-7}$ & .1109 & .1977 & .3034 & .4632 & $H_{3-7}$ & .2920 & .5308 & .7556 & .9427 \\
\hline$H_{3-8}$ & .1084 & .1897 & .2881 & .4418 & $H_{3-8}$ & .2813 & .5113 & .7372 & .9323 \\
\hline$H_{3-9}$ & .1045 & .1862 & .2815 & .4264 & $H_{3-9}$ & .2702 & .4976 & .7227 & .9232 \\
\hline$H_{3-10}$ & .0985 & .1788 & .2731 & .4127 & $H_{3-10}$ & .2579 & .4810 & .7068 & .9155 \\
\hline $\mathrm{KS}$ & .0003 & .0005 & .0005 & .0011 & $\mathrm{KS}$ & .0047 & .0093 & .0214 & .0698 \\
\hline M-KS & .0664 & .0832 & .1074 & .1556 & M-KS & .2003 & .3081 & .4624 & .7087 \\
\hline JB & .1339 & .2179 & .3284 & .5013 & $\mathrm{JB}$ & .3448 & .5826 & .8009 & .9605 \\
\hline
\end{tabular}

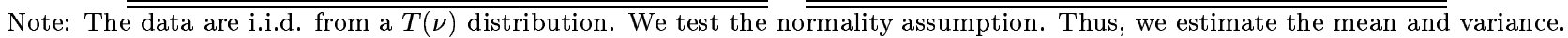
The results are based on 50000 replications. For each sample size, we provide the frequency of rejection at a $5 \%$ level. $H_{i-j}$ is the joint test based on $H_{k}, i \leq k \leq j$. KS, M-KS and JB are the Kolmogorov-Smirnov, modified KS and Jarque-Bera tests. 
Table 3. Continued.

\begin{tabular}{|c|c|c|c|c|c|c|c|c|c|}
\hline \multicolumn{5}{|c|}{ Panel E: $\nu=6$} & \multicolumn{5}{|c|}{ Panel F: $\nu=3$} \\
\hline $\mathrm{T}$ & 100 & 250 & 500 & 1000 & $\mathrm{~T}$ & 100 & 250 & 500 & 1000 \\
\hline$H_{3}$ & .3143 & .4085 & .4654 & .5183 & $H_{3}$ & .6279 & .7567 & .8251 & .8753 \\
\hline$H_{4}$ & .5060 & .8449 & .9802 & .9999 & $H_{4}$ & .8975 & .9983 & 1.000 & 1.000 \\
\hline$H_{5}$ & .3321 & .5637 & .7138 & .8223 & $H_{5}$ & .6973 & .9041 & .9633 & .9849 \\
\hline$H_{6}$ & .2710 & .5219 & .7284 & .8943 & $H_{6}$ & .6649 & .9199 & .9903 & .9998 \\
\hline$H_{7}$ & .2080 & .4501 & .6682 & .8445 & $H_{7}$ & .5831 & .8882 & .9780 & .9959 \\
\hline$H_{8}$ & .1808 & .3894 & .6078 & .8153 & $H_{8}$ & .5339 & .8583 & .9770 & .9991 \\
\hline$H_{9}$ & .2496 & .3968 & .5646 & .7597 & $H_{9}$ & .5982 & .8432 & .9640 & .9961 \\
\hline$H_{10}$ & .2278 & .4615 & .6545 & .8253 & $H_{10}$ & .6109 & .8973 & .9792 & .9971 \\
\hline$H_{3-4}$ & .5117 & .8296 & .9738 & .9998 & $H_{3-4}$ & .8927 & .9976 & 1.000 & 1.000 \\
\hline$H_{3-5}$ & .5013 & .8183 & .9695 & .9995 & $H_{3-5}$ & .8842 & .9967 & 1.000 & 1.000 \\
\hline$H_{3-6}$ & .4946 & .8203 & .9710 & .9997 & $H_{3-6}$ & .8935 & .9979 & 1.000 & 1.000 \\
\hline$H_{3-7}$ & .4766 & .8028 & .9653 & .9994 & $H_{3-7}$ & .8824 & .9970 & 1.000 & 1.000 \\
\hline$H_{3-8}$ & .4650 & .7881 & .9601 & .9993 & $H_{3-8}$ & .8742 & .9966 & 1.000 & 1.000 \\
\hline$H_{3-9}$ & .4540 & .7794 & .9563 & .9991 & $H_{3-9}$ & .8673 & .9960 & 1.000 & 1.000 \\
\hline$H_{3-10}$ & .4404 & .7659 & .9512 & .9989 & $H_{3-10}$ & .8578 & .9956 & 1.000 & 1.000 \\
\hline $\mathrm{KS}$ & .0110 & .0338 & .1152 & .4167 & $\mathrm{KS}$ & .2398 & .6816 & .9726 & 1.000 \\
\hline M-KS & .2450 & .4787 & .7661 & .9701 & M-KS & .7381 & .9789 & .9999 & 1.000 \\
\hline JB & .5195 & .8322 & .9742 & .9998 & $\mathrm{JB}$ & .8961 & .9977 & 1.000 & 1.000 \\
\hline
\end{tabular}

Table 4: Probability of rejection for Student distributions.

\begin{tabular}{ccc}
\hline \hline$\nu$ & $A(\nu)$ & $P\left(A(\nu) \chi^{2}(1)>3.84\right)$ \\
\hline 3 & - & 1 \\
6 & - & 1 \\
10 & 4.16 & .337 \\
20 & 1.74 & .137 \\
30 & 1.41 & .099 \\
60 & 1.17 & .071 \\
\hline
\end{tabular}


Table 5: Power of the tests against asymmetric distributions.

\begin{tabular}{llllllllllll}
\hline \hline \multicolumn{3}{c}{ Panel A: $\chi^{2}(1)$} & distribution. & & \multicolumn{5}{c}{ Panel B: } & exp(1) & distribution. \\
\hline \hline & & 100 & 250 & 500 & 1000 & & T & 100 & 250 & 500 & 1000 \\
$H_{3}$ & 1.000 & 1.000 & 1.000 & 1.000 & & $H_{3}$ & 1.000 & 1.000 & 1.000 & 1.000 \\
$H_{4}$ & .9887 & 1.000 & 1.000 & 1.000 & & $H_{4}$ & .8964 & .9980 & 1.000 & 1.000 \\
$H_{5}$ & .8423 & .9779 & .9993 & 1.000 & & $H_{5}$ & .7001 & .8755 & .9699 & .9981 \\
$H_{6}$ & .8214 & .9855 & .9997 & 1.000 & & $H_{6}$ & .6161 & .9094 & .9909 & .9999 \\
$H_{7}$ & .8620 & .9970 & 1.000 & 1.000 & & $H_{7}$ & .6281 & .9434 & .9977 & 1.000 \\
$H_{8}$ & .7812 & .9593 & .9952 & 1.000 & & $H_{8}$ & .6366 & .8766 & .9533 & .9924 \\
$H_{9}$ & .9162 & .9720 & .9926 & .9994 & & $H_{9}$ & .7427 & .9316 & .9630 & .9871 \\
$H_{10}$ & .7859 & .9400 & .9879 & .9988 & & $H_{10}$ & .6159 & .7908 & .9102 & .9762 \\
$H_{3-4}$ & 1.000 & 1.000 & 1.000 & 1.000 & & $H_{3-4}$ & 1.000 & 1.000 & 1.000 & 1.000 \\
$H_{3-5}$ & 1.000 & 1.000 & 1.000 & 1.000 & & $H_{3-5}$ & 1.000 & 1.000 & 1.000 & 1.000 \\
$H_{3-6}$ & 1.000 & 1.000 & 1.000 & 1.000 & & $H_{3-6}$ & 1.000 & 1.000 & 1.000 & 1.000 \\
$H_{3-7}$ & 1.000 & 1.000 & 1.000 & 1.000 & & $H_{3-7}$ & 1.000 & 1.000 & 1.000 & 1.000 \\
$H_{3-8}$ & 1.000 & 1.000 & 1.000 & 1.000 & & $H_{3-8}$ & 1.000 & 1.000 & 1.000 & 1.000 \\
$H_{3-9}$ & 1.000 & 1.000 & 1.000 & 1.000 & & $H_{3-9}$ & .9999 & 1.000 & 1.000 & 1.000 \\
$H_{3-10}$ & 1.000 & 1.000 & 1.000 & 1.000 & & $H_{3-10}$ & .9999 & 1.000 & 1.000 & 1.000 \\
$\mathrm{KS}$ & 1.000 & 1.000 & 1.000 & 1.000 & & $\mathrm{KS}$ & .9191 & 1.000 & 1.000 & 1.000 \\
$\mathrm{M}-\mathrm{KS}$ & 1.000 & 1.000 & 1.000 & 1.000 & & $\mathrm{M}-\mathrm{KS}$ & 1.000 & 1.000 & 1.000 & 1.000 \\
$\mathrm{JB}$ & 1.000 & 1.000 & 1.000 & 1.000 & & $\mathrm{JB}$ & 1.000 & 1.000 & 1.000 & 1.000 \\
\hline \hline
\end{tabular}

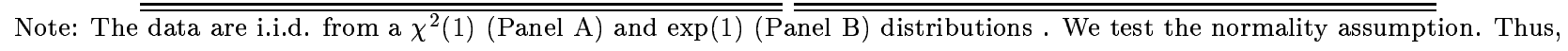
we estimate the mean and variance. The results are based on 50000 replications. For each sample size, we provide the frequency of rejection at a $5 \%$ level. $H_{i-j}$ is the joint test based on $H_{k}, i \leq k \leq j . \mathrm{KS}, \mathrm{M}-\mathrm{KS}$ and JB are the Kolmogorov-Smirnov, modified KS and Jarque-Bera tests. 
Table 6a: Size of the tests under serial correlation which is ignored.

\begin{tabular}{|c|c|c|c|c|c|c|c|c|c|}
\hline \multicolumn{5}{|c|}{ Panel A: $\rho=.1$} & \multicolumn{5}{|c|}{ Panel B: $\rho=.5$} \\
\hline $\mathrm{T}$ & 100 & 250 & 500 & 1000 & $\mathrm{~T}$ & 100 & 250 & 500 & 1000 \\
\hline$H_{3}$ & .0415 & .0466 & .0480 & .0501 & $H_{3}$ & .0595 & .0735 & .0773 & .0812 \\
\hline$H_{4}$ & .0300 & .0393 & .0421 & .0451 & $H_{4}$ & .0266 & .0415 & .0502 & .0568 \\
\hline$H_{5}$ & .0234 & .0352 & .0424 & .0479 & $H_{5}$ & .0199 & .0344 & .0433 & .0491 \\
\hline$H_{6}$ & .0119 & .0214 & .0283 & .0354 & $H_{6}$ & .0093 & .0193 & .0261 & .0333 \\
\hline$H_{7}$ & .0063 & .0121 & .0167 & .0218 & $H_{7}$ & .0058 & .0118 & .0156 & .0210 \\
\hline$H_{8}$ & .0076 & .0092 & .0103 & .0141 & $H_{8}$ & .0068 & .0085 & .0100 & .0127 \\
\hline$H_{9}$ & .0111 & .0134 & .0109 & .0111 & $H_{9}$ & .0084 & .0124 & .0108 & .0111 \\
\hline$H_{10}$ & .0058 & .0128 & .0162 & .0147 & $H_{10}$ & .0043 & .0115 & .0147 & .0152 \\
\hline$H_{3-4}$ & .0390 & .0458 & .0454 & .0474 & $H_{3-4}$ & .0465 & .0606 & .0686 & .0750 \\
\hline$H_{3-5}$ & .0409 & .0518 & .0531 & .0540 & $H_{3-5}$ & .0455 & .0625 & .0701 & .0753 \\
\hline$H_{3-6}$ & .0357 & .0473 & .0519 & .0548 & $H_{3-6}$ & .0388 & .0555 & .0652 & .0712 \\
\hline$H_{3-7}$ & .0323 & .0420 & .0462 & .0513 & $H_{3-7}$ & .0342 & .0481 & .0567 & .0633 \\
\hline$H_{3-8}$ & .0316 & .0397 & .0426 & .0468 & $H_{3-8}$ & .0325 & .0445 & .0509 & .0567 \\
\hline$H_{3-9}$ & .0303 & .0392 & .0420 & .0446 & $H_{3-9}$ & .0293 & .0418 & .0481 & .0521 \\
\hline$H_{3-10}$ & .0279 & .0362 & .0405 & .0436 & $H_{3-10}$ & .0259 & .0383 & .0449 & .0490 \\
\hline $\mathrm{KS}$ & .0001 & .0001 & .0002 & .0002 & $\mathrm{KS}$ & .0004 & .0002 & .0004 & .0004 \\
\hline $\mathrm{M}-\mathrm{KS}$ & .0521 & .0560 & .0562 & .0594 & $\mathrm{M}-\mathrm{KS}$ & .0644 & .0713 & .0744 & .0758 \\
\hline JB & .0413 & .0473 & .0463 & .0479 & JB & .0493 & .0623 & .0696 & .0755 \\
\hline \multicolumn{5}{|c|}{ Panel C: $\rho=.7$} & \multicolumn{5}{|c|}{ Panel D: $\rho=.9$} \\
\hline $\mathrm{T}$ & 100 & 250 & 500 & 1000 & $\mathrm{~T}$ & 100 & 250 & 500 & 1000 \\
\hline$H_{3}$ & .0972 & .1348 & .1497 & .1611 & $H_{3}$ & .1920 & .3009 & .3595 & .3906 \\
\hline$H_{4}$ & .0297 & .0593 & .0854 & .0992 & $H_{4}$ & .0789 & .1964 & .2617 & .3030 \\
\hline$H_{5}$ & .0233 & .0366 & .0530 & .0646 & $H_{5}$ & .1134 & .1373 & .1599 & .1794 \\
\hline$H_{6}$ & .0119 & .0198 & .0298 & .0394 & $H_{6}$ & .0667 & .0959 & .1111 & .1265 \\
\hline$H_{7}$ & .0062 & .0129 & .0175 & .0232 & $H_{7}$ & .0337 & .0513 & .0730 & .0917 \\
\hline$H_{8}$ & .0063 & .0102 & .0123 & .0160 & $H_{8}$ & .0228 & .0378 & .0534 & .0694 \\
\hline$H_{9}$ & .0059 & .0104 & .0125 & .0145 & $H_{9}$ & .0102 & .0256 & .0377 & .0509 \\
\hline$H_{10}$ & .0032 & .0073 & .0126 & .0145 & $H_{10}$ & .0077 & .0179 & .0263 & .0362 \\
\hline$H_{3-4}$ & .0655 & .1067 & .1395 & .1586 & $H_{3-4}$ & .1519 & .3401 & .4423 & .4984 \\
\hline$H_{3-5}$ & .0692 & .1044 & .1306 & .1493 & $H_{3-5}$ & .1983 & .3351 & .4207 & .4825 \\
\hline$H_{3-6}$ & .0582 & .0909 & .1161 & .1354 & $H_{3-6}$ & .2117 & .3423 & .4167 & .4693 \\
\hline$H_{3-7}$ & .0515 & .0788 & .1016 & .1179 & $H_{3-7}$ & .1964 & .3196 & .3983 & .4600 \\
\hline$H_{3-8}$ & .0454 & .0698 & .0912 & .1038 & $H_{3-8}$ & .1849 & .2994 & .3759 & .4385 \\
\hline$H_{3-9}$ & .0392 & .0621 & .0819 & .0939 & $H_{3-9}$ & .1645 & .2780 & .3558 & .4184 \\
\hline$H_{3-10}$ & .0353 & .0555 & .0736 & .0849 & $H_{3-10}$ & .1490 & .2547 & .3328 & .3958 \\
\hline $\mathrm{KS}$ & .0012 & .0015 & .0018 & .0019 & $\mathrm{KS}$ & .0310 & .0506 & .0606 & .0631 \\
\hline M-KS & .1082 & .1199 & .1275 & .1328 & M-KS & .3212 & .3971 & .4298 & .4477 \\
\hline JB & .0694 & .1090 & .1408 & .1595 & JB & .1621 & .3465 & .4457 & .4998 \\
\hline
\end{tabular}

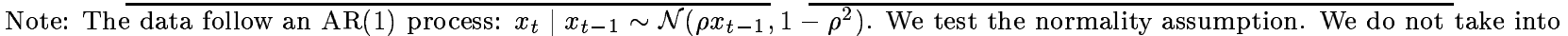
account the serial correlation in the tests. The results are based on 50000 replications. For each sample size, we provide the frequency of rejection at a $5 \%$ level. The notations $H_{i-j}, \mathrm{KS}, \mathrm{M}-\mathrm{KS}$ and JB are defined in Table 5 . 
Table $6 \mathrm{~b}$ : Size of the tests under serial correlation.

The serial correlation is known and taken into account; $\rho$ is known.

\begin{tabular}{|c|c|c|c|c|c|c|c|c|c|}
\hline \multicolumn{5}{|c|}{ Panel A: $\rho=.1$} & \multicolumn{5}{|c|}{ Panel B: $\rho=.5$} \\
\hline $\mathrm{T}$ & 100 & 250 & 500 & 1000 & $\mathrm{~T}$ & 100 & 250 & 500 & 1000 \\
\hline$H_{3}$ & .0409 & .0462 & .0477 & .0498 & $H_{3}$ & .0346 & .0429 & .0456 & .0477 \\
\hline$H_{4}$ & .0300 & .0392 & .0420 & .0451 & $H_{4}$ & .0225 & .0331 & .0361 & .0435 \\
\hline $\mathrm{H}_{5}$ & .0234 & .0352 & .0424 & .0479 & $H_{5}$ & .0167 & .0308 & .0376 & .0455 \\
\hline$H_{6}$ & .0119 & .0214 & .0283 & .0354 & $H_{6}$ & .0091 & .0183 & .0256 & .0335 \\
\hline$H_{7}$ & .0063 & .0121 & .0167 & .0218 & $H_{7}$ & .0050 & .0108 & .0149 & .0211 \\
\hline$H_{8}$ & .0076 & .0092 & .0103 & .0141 & $H_{8}$ & .0064 & .0083 & .0108 & .0128 \\
\hline$H_{9}$ & .0111 & .0134 & .0109 & .0111 & $H_{9}$ & .0082 & .0115 & .0113 & .0110 \\
\hline$H_{10}$ & .0058 & .0128 & .0162 & .0147 & $H_{10}$ & .0045 & .0104 & .0147 & .0138 \\
\hline$H_{3-4}$ & .0388 & .0455 & .0452 & .0472 & $H_{3-4}$ & .0309 & .0405 & .0424 & .0469 \\
\hline$H_{3-5}$ & .0406 & .0517 & .0529 & .0540 & $H_{3-5}$ & .0318 & .0445 & .0482 & .0533 \\
\hline$H_{3-6}$ & .0355 & .0472 & .0517 & .0548 & $H_{3-6}$ & .0276 & .0403 & .0475 & .0541 \\
\hline$H_{3-7}$ & .0322 & .0419 & .0461 & .0511 & $H_{3-7}$ & .0251 & .0365 & .0421 & .0496 \\
\hline$H_{3-8}$ & .0316 & .0397 & .0426 & .0467 & $H_{3-8}$ & .0246 & .0346 & .0395 & .0458 \\
\hline$H_{3-9}$ & .0302 & .0391 & .0419 & .0445 & $H_{3-9}$ & .0230 & .0337 & .0387 & .0439 \\
\hline$H_{3-10}$ & .0277 & .0361 & .0404 & .0435 & $H_{3-10}$ & .0212 & .0316 & .0372 & .0424 \\
\hline \multicolumn{5}{|c|}{ Panel C: $\rho=.7$} & \multicolumn{5}{|c|}{ Panel D: $\rho=.9$} \\
\hline $\mathrm{T}$ & 100 & 250 & 500 & 1000 & $\mathrm{~T}$ & 100 & 250 & 500 & 1000 \\
\hline$H_{3}$ & .0265 & .0388 & .0415 & .0444 & $H_{3}$ & .0090 & .0209 & .0322 & .0405 \\
\hline$H_{4}$ & .0143 & .0257 & .0346 & .0364 & $H_{4}$ & .0040 & .0106 & .0187 & .0264 \\
\hline $\mathrm{H}_{5}$ & .0099 & .0210 & .0308 & .0391 & $H_{5}$ & .0030 & .0073 & .0135 & .0217 \\
\hline$H_{6}$ & .0055 & .0130 & .0196 & .0271 & $H_{6}$ & .0015 & .0035 & .0082 & .0130 \\
\hline$H_{7}$ & .0036 & .0081 & .0115 & .0173 & $H_{7}$ & .0010 & .0025 & .0064 & .0090 \\
\hline$H_{8}$ & .0044 & .0075 & .0096 & .0116 & $H_{8}$ & .0013 & .0032 & .0067 & .0084 \\
\hline$H_{9}$ & .0049 & .0086 & .0104 & .0112 & $H_{9}$ & .0010 & .0034 & .0065 & .0098 \\
\hline$H_{10}$ & .0027 & .0080 & .0115 & .0134 & $H_{10}$ & .0008 & .0021 & .0046 & .0084 \\
\hline$H_{3-4}$ & .0236 & .0349 & .0395 & .0410 & $H_{3-4}$ & .0083 & .0187 & .0298 & .0370 \\
\hline$H_{3-5}$ & .0222 & .0363 & .0441 & .0471 & $H_{3-5}$ & .0068 & .0168 & .0290 & .0379 \\
\hline$H_{3-6}$ & .0184 & .0328 & .0416 & .0455 & $H_{3-6}$ & .0060 & .0135 & .0250 & .0336 \\
\hline$H_{3-7}$ & .0170 & .0296 & .0374 & .0419 & $H_{3-7}$ & .0053 & .0129 & .0231 & .0307 \\
\hline$H_{3-8}$ & .0162 & .0283 & .0350 & .0388 & $H_{3-8}$ & .0047 & .0117 & .0219 & .0296 \\
\hline$H_{3-9}$ & .0146 & .0272 & .0341 & .0376 & $H_{3-9}$ & .0040 & .0107 & .0206 & .0277 \\
\hline$H_{3-10}$ & .0133 & .0254 & .0321 & .0364 & $H_{3-10}$ & .0035 & .0097 & .0197 & .0267 \\
\hline
\end{tabular}

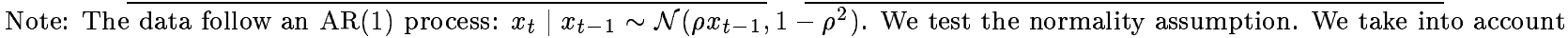
the serial correlation in the tests. We assume that we know the AR(1) dynamics and that we know $\rho$. The results are based on 50000 replications. For each sample size, we provide the frequency of rejection at a $5 \%$ level. The notations $H_{i-j}$ are defined in Table 5. 
Table 6c: Size of the tests under serial correlation.

The serial correlation is known and taken into account; $\rho$ is estimated.

\begin{tabular}{|c|c|c|c|c|c|c|c|c|c|}
\hline \multicolumn{5}{|c|}{ Panel A: $\rho=.1$} & \multicolumn{5}{|c|}{ Panel B: $\rho=.5$} \\
\hline$\overline{\mathrm{T}}$ & 100 & 250 & 500 & 1000 & $\mathrm{~T}$ & 100 & 250 & 500 & 1000 \\
\hline$H_{3}$ & .0412 & .0464 & .0479 & .0499 & $H_{3}$ & .0339 & .0430 & .0459 & .0478 \\
\hline$H_{4}$ & .0300 & .0393 & .0420 & .0451 & $H_{4}$ & .0221 & .0326 & .0364 & .0435 \\
\hline$H_{5}$ & .0234 & .0352 & .0424 & .0479 & $H_{5}$ & .0165 & .0306 & .0378 & .0455 \\
\hline$H_{6}$ & .0119 & .0214 & .0283 & .0354 & $H_{6}$ & .0092 & .0184 & .0257 & .0334 \\
\hline$H_{7}$ & .0063 & .0121 & .0167 & .0218 & $H_{7}$ & .0051 & .0108 & .0149 & .0211 \\
\hline$H_{8}$ & .0076 & .0092 & .0103 & .0141 & $H_{8}$ & .0065 & .0084 & .0108 & .0128 \\
\hline$H_{9}$ & .0111 & .0134 & .0109 & .0111 & $H_{9}$ & .0083 & .0115 & .0113 & .0110 \\
\hline$H_{10}$ & .0058 & .0128 & .0162 & .0147 & $H_{10}$ & .0045 & .0104 & .0147 & .0137 \\
\hline$H_{3-4}$ & .0389 & .0457 & .0453 & .0473 & $H_{3-4}$ & .0315 & .0400 & .0421 & .0472 \\
\hline$H_{3-5}$ & .0408 & .0517 & .0529 & .0539 & $H_{3-5}$ & .0318 & .0444 & .0486 & .0532 \\
\hline$H_{3-6}$ & .0356 & .0473 & .0517 & .0548 & $H_{3-6}$ & .0275 & .0408 & .0473 & .0537 \\
\hline$H_{3-7}$ & .0322 & .0419 & .0460 & .0512 & $H_{3-7}$ & .0245 & .0367 & .0426 & .0500 \\
\hline$H_{3-8}$ & .0316 & .0397 & .0426 & .0467 & $H_{3-8}$ & .0245 & .0350 & .0395 & .0459 \\
\hline$H_{3-9}$ & .0303 & .0392 & .0419 & .0446 & $H_{3-9}$ & .0229 & .0338 & .0388 & .0439 \\
\hline$H_{3-10}$ & .0279 & .0362 & .0404 & .0435 & $H_{3-10}$ & .0211 & .0317 & .0374 & .0425 \\
\hline \multicolumn{5}{|c|}{ Panel C: $\rho=.7$} & \multicolumn{5}{|c|}{ Panel D: $\rho=.9$} \\
\hline $\mathrm{T}$ & 100 & 250 & 500 & 1000 & $\overline{\mathrm{T}}$ & 100 & 250 & 500 & 1000 \\
\hline$H_{3}$ & .0235 & .0376 & .0414 & .0437 & $H_{3}$ & .0023 & .0163 & .0293 & .0381 \\
\hline$H_{4}$ & .0126 & .0243 & .0339 & .0361 & $H_{4}$ & .0016 & .0085 & .0172 & .0258 \\
\hline$H_{5}$ & .0096 & .0204 & .0305 & .0391 & $H_{5}$ & .0020 & .0064 & .0130 & .0213 \\
\hline$H_{6}$ & .0052 & .0130 & .0197 & .0268 & $H_{6}$ & .0005 & .0033 & .0082 & .0130 \\
\hline$H_{7}$ & .0035 & .0080 & .0116 & .0170 & $H_{7}$ & .0007 & .0022 & .0057 & .0090 \\
\hline$H_{8}$ & .0042 & .0074 & .0094 & .0117 & $H_{8}$ & .0006 & .0023 & .0067 & .0080 \\
\hline$H_{9}$ & .0048 & .0087 & .0105 & .0111 & $H_{9}$ & .0005 & .0024 & .0062 & .0094 \\
\hline$H_{10}$ & .0029 & .0079 & .0116 & .0135 & $H_{10}$ & .0005 & .0016 & .0041 & .0079 \\
\hline$H_{3-4}$ & .0204 & .0335 & .0383 & .0400 & $H_{3-4}$ & .0029 & .0144 & .0273 & .0353 \\
\hline$H_{3-5}$ & .0198 & .0359 & .0432 & .0465 & $H_{3-5}$ & .0026 & .0137 & .0268 & .0370 \\
\hline$H_{3-6}$ & .0171 & .0319 & .0411 & .0460 & $H_{3-6}$ & .0021 & .0113 & .0233 & .0334 \\
\hline$H_{3-7}$ & .0157 & .0293 & .0371 & .0419 & $H_{3-7}$ & .0019 & .0105 & .0224 & .0305 \\
\hline$H_{3-8}$ & .0149 & .0284 & .0351 & .0394 & $H_{3-8}$ & .0015 & .0099 & .0210 & .0291 \\
\hline$H_{3-9}$ & .0137 & .0273 & .0336 & .0375 & $H_{3-9}$ & .0015 & .0089 & .0199 & .0278 \\
\hline$H_{3-10}$ & .0125 & .0258 & .0319 & .0364 & $H_{3-10}$ & .0014 & .0082 & .0186 & .0264 \\
\hline
\end{tabular}

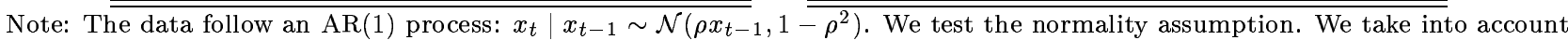
the serial correlation in the tests. We assume that we know the AR(1) dynamics but not $\rho$ which is estimated by OLS. The results are based on 50000 replications. For each sample size, we provide the frequency of rejection at a $5 \%$ level. The notations $H_{i-j}$ are defined in Table 5. 
Table 6d: Size of the tests under serial correlation.

The serial correlation is unknown; $\Sigma$ is estimated by a HAC procedure.

\begin{tabular}{|c|c|c|c|c|c|c|c|c|c|}
\hline \multicolumn{5}{|c|}{ Panel A: $\rho=.1$} & \multicolumn{5}{|c|}{ Panel B: $\rho=.5$} \\
\hline$\overline{\mathrm{T}}$ & 100 & 250 & 500 & 1000 & $\mathrm{~T}$ & 100 & 250 & 500 & 1000 \\
\hline$H_{3}$ & .0338 & .0416 & .0463 & .0477 & $H_{3}$ & .0334 & .0424 & .0464 & .0501 \\
\hline$H_{4}$ & .0556 & .0756 & .0754 & .0694 & $H_{4}$ & .0386 & .0665 & .0732 & .0713 \\
\hline$H_{5}$ & .0404 & .0407 & .0420 & .0415 & $H_{5}$ & .0421 & .0431 & .0428 & .0434 \\
\hline$H_{6}$ & .0441 & .0416 & .0459 & .0497 & $H_{6}$ & .0452 & .0442 & .0463 & .0466 \\
\hline$H_{7}$ & .0372 & .0431 & .0468 & .0469 & $H_{7}$ & .0380 & .0424 & .0442 & .0469 \\
\hline$H_{8}$ & .0387 & .0444 & .0483 & .0494 & $H_{8}$ & .0360 & .0426 & .0466 & .0466 \\
\hline$H_{9}$ & .0402 & .0450 & .0441 & .0453 & $H_{9}$ & .0413 & .0429 & .0443 & .0450 \\
\hline$H_{10}$ & .0400 & .0462 & .0456 & .0455 & $H_{10}$ & .0379 & .0430 & .0449 & .0461 \\
\hline$H_{3-4}$ & .0423 & .0822 & .0894 & .0825 & $H_{3-4}$ & .0276 & .0702 & .0875 & .0879 \\
\hline$H_{3-5}$ & .0186 & .0642 & .1022 & .1090 & $H_{3-5}$ & .0141 & .0424 & .0804 & .1017 \\
\hline$H_{3-6}$ & .0157 & .0356 & .1362 & .2218 & $H_{3-6}$ & .0133 & .0233 & .0648 & .1599 \\
\hline$H_{3-7}$ & .0286 & .0342 & .0971 & .2402 & $H_{3-7}$ & .0292 & .0254 & .0495 & .1393 \\
\hline$H_{3-8}$ & .1267 & .0648 & .0809 & .2682 & $H_{3-8}$ & .1311 & .0593 & .0536 & .1119 \\
\hline$H_{3-9}$ & .2706 & .1249 & .1014 & .2160 & $H_{3-9}$ & .2866 & .1325 & .0828 & .1062 \\
\hline$H_{3-10}$ & .6553 & .3723 & .2440 & .2326 & $H_{3-10}$ & .6810 & .3951 & .2390 & .1703 \\
\hline \multicolumn{5}{|c|}{ Panel C: $\rho=.7$} & \multicolumn{5}{|c|}{ Panel D: $\rho=.9$} \\
\hline$\overline{\mathrm{T}}$ & 100 & 250 & 500 & 1000 & $\mathrm{~T}$ & 100 & 250 & 500 & 1000 \\
\hline$H_{3}$ & .0293 & .0400 & .0436 & .0479 & $H_{3}$ & .0156 & .0258 & .0346 & .0399 \\
\hline$H_{4}$ & .0229 & .0512 & .0708 & .0747 & $H_{4}$ & .0065 & .0182 & .0383 & .0613 \\
\hline$H_{5}$ & .0477 & .0475 & .0470 & .0456 & $H_{5}$ & .0449 & .0504 & .0525 & .0504 \\
\hline$H_{6}$ & .0534 & .0475 & .0463 & .0472 & $H_{6}$ & .0485 & .0638 & .0576 & .0518 \\
\hline$H_{7}$ & .0387 & .0449 & .0459 & .0475 & $H_{7}$ & .0411 & .0416 & .0435 & .0463 \\
\hline$H_{8}$ & .0387 & .0420 & .0452 & .0485 & $H_{8}$ & .0483 & .0467 & .0455 & .0485 \\
\hline$H_{9}$ & .0400 & .0436 & .0445 & .0438 & $H_{9}$ & .0371 & .0445 & .0443 & .0461 \\
\hline$H_{10}$ & .0373 & .0429 & .0461 & .0465 & $H_{10}$ & .0377 & .0436 & .0462 & .0492 \\
\hline$H_{3-4}$ & .0154 & .0461 & .0771 & .0872 & $H_{3-4}$ & .0053 & .0118 & .0283 & .0571 \\
\hline$H_{3-5}$ & .0110 & .0240 & .0483 & .0775 & $H_{3-5}$ & .0047 & .0093 & .0151 & .0284 \\
\hline$H_{3-6}$ & .0128 & .0179 & .0278 & .0656 & $H_{3-6}$ & .0043 & .0131 & .0159 & .0191 \\
\hline$H_{3-7}$ & .0291 & .0234 & .0268 & .0498 & $H_{3-7}$ & .0108 & .0288 & .0301 & .0238 \\
\hline$H_{3-8}$ & .1344 & .0700 & .0459 & .0472 & $H_{3-8}$ & .0322 & .1309 & .1119 & .0635 \\
\hline$H_{3-9}$ & .2868 & .1668 & .0925 & .0640 & $H_{3-9}$ & .0714 & .2551 & .2397 & .1385 \\
\hline$H_{3-10}$ & .6705 & .4716 & .2861 & .1618 & $H_{3-10}$ & .1330 & .5814 & .5634 & .3831 \\
\hline
\end{tabular}

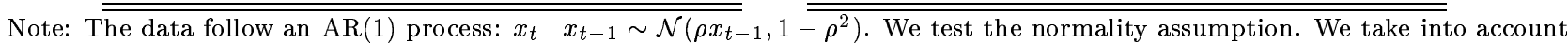
the serial correlation in the tests. We assume that we do not know the AR(1) dynamics. We use a HAC method. The results are based on 50000 replications. For each sample size, we provide the frequency of rejection at a $5 \%$ level. The notations $H_{i-j}$ are defined in Table 5. 
Table 7a: Power of the tests under serial correlation against $T(60)$ innovations.

\begin{tabular}{|c|c|c|c|c|c|c|c|c|c|}
\hline \multicolumn{5}{|c|}{ Panel A: $\rho=.1$} & \multicolumn{5}{|c|}{ Panel B: $\rho=.5$} \\
\hline $\mathrm{T}$ & 100 & 250 & 500 & 1000 & $\mathrm{~T}$ & 100 & 250 & 500 & 1000 \\
\hline$H_{3}$ & .0200 & .0699 & .1198 & .2102 & $H_{3}$ & .0071 & .0223 & .0471 & .0957 \\
\hline$H_{4}$ & .8742 & .8853 & .9016 & .8263 & $H_{4}$ & .9173 & .9253 & .9305 & .7719 \\
\hline$H_{5}$ & .8902 & .8944 & .8962 & .6667 & $H_{5}$ & .9293 & .9352 & .9362 & .6891 \\
\hline$H_{6}$ & .8844 & .8930 & .9049 & .6985 & $H_{6}$ & .9274 & .9347 & .9385 & .7109 \\
\hline$H_{7}$ & .8772 & .8839 & .8925 & .6633 & $H_{7}$ & .9234 & .9311 & .9343 & .6728 \\
\hline$H_{8}$ & .8670 & .8749 & .8820 & .6002 & $H_{8}$ & .9178 & .9267 & .9300 & .6267 \\
\hline$H_{9}$ & .8566 & .8669 & .8732 & .5557 & $H_{9}$ & .9114 & .9224 & .9262 & .5713 \\
\hline$H_{10}$ & .8532 & .8619 & .8680 & .5285 & $H_{10}$ & .9079 & .9186 & .9227 & .5396 \\
\hline$H_{3-4}$ & .0297 & .0574 & .0843 & .1185 & $H_{3-4}$ & .0124 & .0236 & .0388 & .0826 \\
\hline$H_{3-5}$ & .0037 & .0039 & .0083 & .0627 & $H_{3-5}$ & .0013 & .0018 & .0026 & .0287 \\
\hline$H_{3-6}$ & .8434 & .8620 & .8638 & .4546 & $H_{3-6}$ & .8968 & .9155 & .9194 & .4587 \\
\hline$H_{3-7}$ & .8665 & .8763 & .8871 & .5990 & $H_{3-7}$ & .9168 & .9270 & .9307 & .6120 \\
\hline$H_{3-8}$ & .8664 & .8737 & .8803 & .5929 & $H_{3-8}$ & .9168 & .9263 & .9292 & .6077 \\
\hline$H_{3-9}$ & .8591 & .8679 & .8741 & .5539 & $H_{3-9}$ & .9131 & .9236 & .9269 & .5803 \\
\hline$H_{3-10}$ & .8501 & .8611 & .8669 & .5062 & $H_{3-10}$ & .9075 & .9194 & .9235 & .5312 \\
\hline & Par & $1 \mathrm{C}: \rho$ & $=.7$ & & & $\overline{\operatorname{Pan}}$ & $1 \mathrm{D}: \rho$ & $=.9$ & \\
\hline $\mathrm{T}$ & 100 & 250 & 500 & 1000 & $\bar{T}$ & 100 & 250 & 500 & 1000 \\
\hline$H_{3}$ & .0019 & .0056 & .0114 & .0330 & $H_{3}$ & .0002 & .0007 & .0008 & .0014 \\
\hline$H_{4}$ & .9414 & .9493 & .9525 & .7186 & $H_{4}$ & .0025 & .9756 & .9815 & .0401 \\
\hline$H_{5}$ & .9510 & .9570 & .9584 & .7218 & $H_{5}$ & .0017 & .9793 & .9848 & .0359 \\
\hline$H_{6}$ & .9511 & .9573 & .9598 & .7137 & $H_{6}$ & .0057 & .9800 & .9850 & .0634 \\
\hline$H_{7}$ & .9487 & .9555 & .9584 & .6979 & $H_{7}$ & .0208 & .9796 & .9846 & .0862 \\
\hline$H_{8}$ & .9449 & .9532 & .9563 & .6617 & $H_{8}$ & .0018 & .9782 & .9839 & .0355 \\
\hline$H_{9}$ & .9392 & .9506 & .9541 & .6043 & $H_{9}$ & .0002 & .9764 & .9830 & .0126 \\
\hline$H_{10}$ & .9331 & .9480 & .9521 & .5406 & $H_{10}$ & .0002 & .9742 & .9821 & .0060 \\
\hline$H_{3-4}$ & .0064 & .0093 & .0138 & .0500 & $H_{3-4}$ & .0055 & .0025 & .0021 & .0376 \\
\hline$H_{3-5}$ & .0006 & .0012 & .0013 & .0125 & $H_{3-5}$ & .0001 & .0002 & .0004 & .0017 \\
\hline$H_{3-6}$ & .9123 & .9438 & .9489 & .4083 & $H_{3-6}$ & .0014 & .9548 & .9783 & .0077 \\
\hline$H_{3-7}$ & .9421 & .9526 & .9562 & .6140 & $H_{3-7}$ & .0004 & .9764 & .9832 & .0143 \\
\hline$H_{3-8}$ & .9434 & .9526 & .9561 & .6371 & $H_{3-8}$ & .0013 & .9773 & .9834 & .0285 \\
\hline$H_{3-9}$ & .9404 & .9511 & .9546 & .6142 & $H_{3-9}$ & .0004 & .9764 & .9830 & .0163 \\
\hline$H_{3-10}$ & .9352 & .9488 & .9529 & .5630 & $H_{3-10}$ & .0002 & .9750 & .9824 & .0078 \\
\hline
\end{tabular}

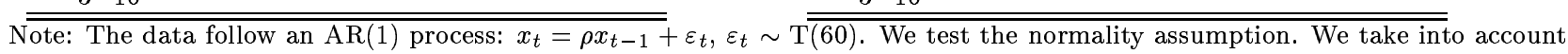
the serial correlation in the tests. We assume that we do not know the AR(1) dynamics. We use a HAC method. The results are based on 50000 replications. For each sample size, we provide the frequency of rejection at a $5 \%$ level. The notations $H_{i-j}$ are defined in Table 5. 
Table $7 \mathrm{~b}$ : Power of the tests under serial correlation against $T(30)$ innovations.

\begin{tabular}{|c|c|c|c|c|c|c|c|c|c|}
\hline \multirow{2}{*}{\multicolumn{5}{|c|}{ Panel A: $\rho=.1$}} & \multirow{2}{*}{\multicolumn{5}{|c|}{ Panel B: $\rho=.5$}} \\
\hline & & & & & & & & & \\
\hline $\mathrm{T}$ & 100 & 250 & 500 & 1000 & $\overline{\mathrm{T}}$ & 100 & 250 & 500 & 1000 \\
\hline$H_{3}$ & .0497 & .1404 & .2063 & .2465 & $H_{3}$ & .0161 & .0512 & .0932 & .1607 \\
\hline$H_{4}$ & .8225 & .8693 & .9181 & .9494 & $H_{4}$ & .8811 & .9009 & .9180 & .8512 \\
\hline$H_{5}$ & .8406 & .8529 & .8586 & .7845 & $H_{5}$ & .8979 & .9072 & .9085 & .6610 \\
\hline$H_{6}$ & .8278 & .8615 & .8959 & .8228 & $H_{6}$ & .8935 & .9097 & .9210 & .7100 \\
\hline$H_{7}$ & .8161 & .8442 & .8681 & .8110 & $H_{7}$ & .8881 & .9021 & .9105 & .6877 \\
\hline$H_{8}$ & .7995 & .8261 & .8491 & .7411 & $H_{8}$ & .8783 & .8930 & .9014 & .6167 \\
\hline$H_{9}$ & .7829 & .8114 & .8325 & .7081 & $H_{9}$ & .8674 & .8847 & .8929 & .5648 \\
\hline$H_{10}$ & .7750 & .8026 & .8195 & .6710 & $H_{10}$ & .8600 & .8787 & .8860 & .5293 \\
\hline$H_{3-4}$ & .0576 & .0939 & .1286 & .0756 & $H_{3-4}$ & .0249 & .0434 & .0657 & .0880 \\
\hline$H_{3-5}$ & .0048 & .0108 & .0356 & .0893 & $H_{3-5}$ & .0021 & .0031 & .0070 & .0730 \\
\hline$H_{3-6}$ & .7671 & .7947 & .7928 & .5632 & $H_{3-6}$ & .8460 & .8727 & .8759 & .4129 \\
\hline$H_{3-7}$ & .7990 & .8309 & .8638 & .7403 & $H_{3-7}$ & .8761 & .8928 & .9051 & .6047 \\
\hline$H_{3-8}$ & .7988 & .8217 & .8429 & .7462 & $H_{3-8}$ & .8764 & .8916 & .8989 & .6119 \\
\hline$H_{3-9}$ & .7866 & .8122 & .8331 & .6941 & $H_{3-9}$ & .8697 & .8859 & .8941 & .5666 \\
\hline$H_{3-10}$ & .7720 & .8000 & .8161 & .6363 & $H_{3-10}$ & .8597 & .8795 & .8869 & .5081 \\
\hline \multicolumn{5}{|c|}{ Panel C: $\rho=.7$} & \multicolumn{5}{|c|}{ Panel D: $\rho=.9$} \\
\hline $\mathrm{T}$ & 100 & 250 & 500 & 1000 & $\mathrm{~T}$ & 100 & 250 & 500 & 1000 \\
\hline$H_{3}$ & .0044 & .0129 & .0296 & .0704 & $H_{3}$ & .0004 & .0007 & .0013 & .0036 \\
\hline$H_{4}$ & .9120 & .9269 & .9351 & .6338 & $H_{4}$ & .0072 & .9669 & .9717 & .0465 \\
\hline$H_{5}$ & .9269 & .9374 & .9416 & .5083 & $H_{5}$ & .0037 & .9727 & .9765 & .0491 \\
\hline$H_{6}$ & .9265 & .9380 & .9444 & .5239 & $H_{6}$ & .0152 & .9736 & .9772 & .0609 \\
\hline$H_{7}$ & .9234 & .9350 & .9415 & .5138 & $H_{7}$ & .0427 & .9729 & .9765 & .0624 \\
\hline$H_{8}$ & .9170 & .9306 & .9377 & .4549 & $H_{8}$ & .0050 & .9709 & .9751 & .0378 \\
\hline$H_{9}$ & .9082 & .9266 & .9339 & .3856 & $H_{9}$ & .0007 & .9678 & .9739 & .0193 \\
\hline$H_{10}$ & .8977 & .9222 & .9299 & .3371 & $H_{10}$ & .0003 & .9639 & .9723 & .0135 \\
\hline$H_{3-4}$ & .0123 & .0173 & .0285 & .0848 & $H_{3-4}$ & .0081 & .0034 & .0037 & .0942 \\
\hline$H_{3-5}$ & .0008 & .0017 & .0019 & .0586 & $H_{3-5}$ & .0001 & .0003 & .0004 & .0163 \\
\hline$H_{3-6}$ & .8597 & .9145 & .9244 & .2423 & $H_{3-6}$ & .0020 & .9243 & .9672 & .0218 \\
\hline$H_{3-7}$ & .9123 & .9293 & .9376 & .4066 & $H_{3-7}$ & .0010 & .9676 & .9741 & .0391 \\
\hline$H_{3-8}$ & .9145 & .9295 & .9367 & .4394 & $H_{3-8}$ & .0032 & .9694 & .9747 & .0324 \\
\hline$H_{3-9}$ & .9098 & .9272 & .9346 & .3989 & $H_{3-9}$ & .0012 & .9681 & .9739 & .0246 \\
\hline$H_{3-10}$ & .9023 & .9237 & .9316 & .3473 & $H_{3-10}$ & .0003 & .9654 & .9728 & .0149 \\
\hline
\end{tabular}

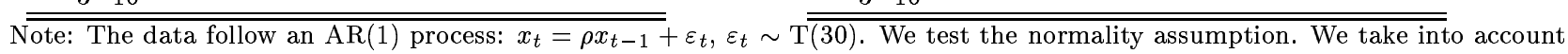
the serial correlation in the tests. We assume that we do not know the AR(1) dynamics. We use a HAC method. The results are based on 50000 replications. For each sample size, we provide the frequency of rejection at a $5 \%$ level. The notations $H_{i-j}$ are defined in Table 5. 
Table 7c: Power of the tests under serial correlation against $T(20)$ innovations.

\begin{tabular}{|c|c|c|c|c|c|c|c|c|c|}
\hline \multicolumn{5}{|c|}{ Panel A: $\rho=.1$} & \multicolumn{5}{|c|}{ Panel B: $\rho=.5$} \\
\hline $\mathrm{T}$ & 100 & 250 & 500 & 1000 & $\mathrm{~T}$ & 100 & 250 & 500 & 1000 \\
\hline$H_{3}$ & .0790 & .1961 & .2767 & .3733 & $H_{3}$ & .0262 & .0781 & .1322 & .1828 \\
\hline$H_{4}$ & .7912 & .8757 & .9494 & .9932 & $H_{4}$ & .8508 & .8902 & .9243 & .9676 \\
\hline$H_{5}$ & .7981 & .8185 & .8396 & .8701 & $H_{5}$ & .8701 & .8872 & .8884 & .8972 \\
\hline$H_{6}$ & .7882 & .8481 & .9053 & .9616 & $H_{6}$ & .8637 & .8962 & .9163 & .9465 \\
\hline$H_{7}$ & .7689 & .8189 & .8673 & .9213 & $H_{7}$ & .8544 & .8832 & .8983 & .9225 \\
\hline$H_{8}$ & .7442 & .7940 & .8418 & .8980 & $H_{8}$ & .8411 & .8727 & .8854 & .9087 \\
\hline$H_{9}$ & .7192 & .7713 & .8185 & .8713 & $H_{9}$ & .8248 & .8598 & .8738 & .8951 \\
\hline$H_{10}$ & .7059 & .7570 & .8004 & .8493 & $H_{10}$ & .8140 & .8499 & .8627 & .8833 \\
\hline$H_{3-4}$ & .0810 & .1161 & .1521 & .2133 & $H_{3-4}$ & .0366 & .0579 & .0836 & .1152 \\
\hline$H_{3-5}$ & .0070 & .0257 & .0896 & .2328 & $H_{3-5}$ & .0028 & .0053 & .0191 & .0663 \\
\hline$H_{3-6}$ & .6958 & .7253 & .7344 & .7578 & $H_{3-6}$ & .7943 & .8371 & .8355 & .8374 \\
\hline$H_{3-7}$ & .7425 & .8036 & .8685 & .9404 & $H_{3-7}$ & .8367 & .8737 & .8941 & .9290 \\
\hline$H_{3-8}$ & .7410 & .7864 & .8316 & .8899 & $H_{3-8}$ & .8375 & .8684 & .8811 & .9044 \\
\hline$H_{3-9}$ & .7229 & .7719 & .8177 & .8722 & $H_{3-9}$ & .8280 & .8619 & .8751 & .8979 \\
\hline$H_{3-10}$ & .7016 & .7516 & .7924 & .8412 & $H_{3-10}$ & .8144 & .8510 & .8643 & .8836 \\
\hline \multicolumn{5}{|c|}{ Panel C: $\rho=.7$} & \multicolumn{5}{|c|}{ Panel D: $\rho=.9$} \\
\hline $\mathrm{T}$ & 100 & 250 & 500 & 1000 & $\mathrm{~T}$ & 100 & 250 & 500 & 1000 \\
\hline$H_{3}$ & .0071 & .0230 & .0499 & .0824 & $H_{3}$ & .0007 & .0013 & .0024 & .0051 \\
\hline$H_{4}$ & .8871 & .9111 & .9223 & .9370 & $H_{4}$ & .0137 & .9559 & .9660 & .9678 \\
\hline$H_{5}$ & .9055 & .9226 & .9257 & .9255 & $H_{5}$ & .0071 & .9641 & .9717 & .9730 \\
\hline$H_{6}$ & .9046 & .9238 & .9328 & .9418 & $H_{6}$ & .0251 & .9652 & .9723 & .9738 \\
\hline$H_{7}$ & .9007 & .9196 & .9269 & .9330 & $H_{7}$ & .0599 & .9641 & .9715 & .9730 \\
\hline$H_{8}$ & .8921 & .9141 & .9218 & .9277 & $H_{8}$ & .0094 & .9612 & .9704 & .9718 \\
\hline$H_{9}$ & .8789 & .9075 & .9168 & .9220 & $H_{9}$ & .0014 & .9566 & .9687 & .9706 \\
\hline$H_{10}$ & .8638 & .9009 & .9103 & .9154 & $H_{10}$ & .0005 & .9516 & .9663 & .9688 \\
\hline$H_{3-4}$ & .0169 & .0272 & .0418 & .0592 & $H_{3-4}$ & .0112 & .0046 & .0052 & .0079 \\
\hline$H_{3-5}$ & .0013 & .0016 & .0031 & .0089 & $H_{3-5}$ & .0001 & .0003 & .0006 & .0006 \\
\hline$H_{3-6}$ & .8050 & .8905 & .9009 & .8998 & $H_{3-6}$ & .0032 & .8901 & .9593 & .9643 \\
\hline$H_{3-7}$ & .8843 & .9119 & .9225 & .9317 & $H_{3-7}$ & .0016 & .9563 & .9687 & .9711 \\
\hline$H_{3-8}$ & .8879 & .9119 & .9204 & .9258 & $H_{3-8}$ & .0067 & .9589 & .9696 & .9712 \\
\hline$H_{3-9}$ & .8812 & .9084 & .9173 & .9227 & $H_{3-9}$ & .0023 & .9570 & .9687 & .9706 \\
\hline$H_{3-10}$ & .8698 & .9029 & .9127 & .9180 & $H_{3-10}$ & .0006 & .9532 & .9670 & .9694 \\
\hline
\end{tabular}

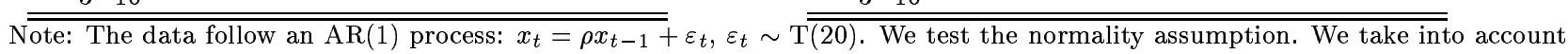
the serial correlation in the tests. We assume that we do not know the AR(1) dynamics. We use a HAC method. The results are based on 50000 replications. For each sample size, we provide the frequency of rejection at a $5 \%$ level. The notations $H_{i-j}$ are defined in Table 5. 
Table $7 \mathrm{~d}$ : Power of the tests under serial correlation against $\mathrm{T}(10)$ innovations.

\begin{tabular}{|c|c|c|c|c|c|c|c|c|c|}
\hline \multirow{2}{*}{\multicolumn{5}{|c|}{ Panel A: $\rho$}} & \multirow{2}{*}{\multicolumn{5}{|c|}{ Panel B: $\rho=.5$}} \\
\hline & & & & & & & & & \\
\hline $\mathrm{T}$ & 100 & 250 & 500 & 1000 & $\mathrm{~T}$ & 100 & 250 & 500 & 1000 \\
\hline$H_{3}$ & .1531 & .3184 & .4744 & .6205 & $H_{3}$ & .0575 & .1500 & .2404 & .3716 \\
\hline$H_{4}$ & .7713 & .9417 & .9946 & .9999 & $H_{4}$ & .7975 & .9017 & .9714 & .9983 \\
\hline$H_{5}$ & .7066 & .7774 & .8496 & .9234 & $H_{5}$ & .7886 & .8184 & .8451 & .8839 \\
\hline$H_{6}$ & .7126 & .8650 & .9565 & .9955 & $H_{6}$ & .7889 & .8729 & .9349 & .9829 \\
\hline$H_{7}$ & .6798 & .8198 & .9162 & .9762 & $H_{7}$ & .7683 & .8419 & .8957 & .9504 \\
\hline$H_{8}$ & .6349 & .7762 & .8839 & .9611 & $H_{8}$ & .7407 & .8185 & .8787 & .9382 \\
\hline$H_{9}$ & .5945 & .7404 & .8535 & .9423 & $H_{9}$ & .7059 & .7934 & .8542 & .9176 \\
\hline$H_{10}$ & .5760 & .7130 & .8226 & .9210 & $H_{10}$ & .6801 & .7729 & .8329 & .8982 \\
\hline$H_{3-4}$ & .1165 & .1302 & .1677 & .2382 & $H_{3-4}$ & .0612 & .0818 & .1082 & .1515 \\
\hline$H_{3-5}$ & .0260 & .1079 & .2833 & .4745 & $H_{3-5}$ & .0094 & .0296 & .1041 & .2614 \\
\hline$H_{3-6}$ & .5396 & .6042 & .6884 & .8079 & $H_{3-6}$ & .6347 & .6939 & .7078 & .7406 \\
\hline$H_{3-7}$ & .6331 & .8015 & .9271 & .9919 & $H_{3-7}$ & .7304 & .8284 & .9060 & .9733 \\
\hline$H_{3-8}$ & .6264 & .7602 & .8734 & .9536 & $H_{3-8}$ & .7302 & .8033 & .8600 & .9213 \\
\hline$H_{3-9}$ & .5979 & .7382 & .8519 & .9463 & $H_{3-9}$ & .7117 & .7967 & .8586 & .9235 \\
\hline$H_{3-10}$ & .5631 & .6960 & .8060 & .9104 & $H_{3-10}$ & .6813 & .7706 & .8287 & .8931 \\
\hline \multicolumn{5}{|c|}{ Panel C: $\rho=.7$} & \multicolumn{5}{|c|}{ Panel D: $\rho=.9$} \\
\hline $\mathrm{T}$ & 100 & 250 & 500 & 1000 & $\mathrm{~T}$ & 100 & 250 & 500 & 1000 \\
\hline$H_{3}$ & .0159 & .0548 & .1023 & .1602 & $H_{3}$ & .0010 & .0024 & .0059 & .0138 \\
\hline$H_{4}$ & .8106 & .8784 & .9210 & .9692 & $H_{4}$ & .0297 & .9276 & .9441 & .9520 \\
\hline$H_{5}$ & .8382 & .8741 & .8809 & .8906 & $H_{5}$ & .0201 & .9412 & .9532 & .9576 \\
\hline$H_{6}$ & .8326 & .8862 & .9188 & .9541 & $H_{6}$ & .0486 & .9427 & .9545 & .9599 \\
\hline$H_{7}$ & .8241 & .8756 & .9009 & .9287 & $H_{7}$ & .0801 & .9415 & .9528 & .9585 \\
\hline$H_{8}$ & .8048 & .8654 & .8904 & .9196 & $H_{8}$ & .0228 & .9368 & .9502 & .9566 \\
\hline$H_{9}$ & .7734 & .8512 & .8776 & .9049 & $H_{9}$ & .0058 & .9278 & .9473 & .9539 \\
\hline$H_{10}$ & .7339 & .8356 & .8636 & .8913 & $H_{10}$ & .0024 & .9167 & .9431 & .9506 \\
\hline$H_{3-4}$ & .0304 & .0478 & .0650 & .0873 & $H_{3-4}$ & .0210 & .0084 & .0111 & .0159 \\
\hline$H_{3-5}$ & .0036 & .0057 & .0178 & .0612 & $H_{3-5}$ & .0003 & .0006 & .0006 & .0010 \\
\hline$H_{3-6}$ & .6122 & .8001 & .8083 & .8070 & $H_{3-6}$ & .0052 & .7310 & .9293 & .9422 \\
\hline$H_{3-7}$ & .7802 & .8615 & .8958 & .9379 & $H_{3-7}$ & .0067 & .9261 & .9470 & .9545 \\
\hline$H_{3-8}$ & .7927 & .8580 & .8813 & .9087 & $H_{3-8}$ & .0178 & .9323 & .9485 & .9552 \\
\hline$H_{3-9}$ & .7788 & .8532 & .8795 & .9095 & $H_{3-9}$ & .0085 & .9284 & .9471 & .9539 \\
\hline$H_{3-10}$ & .7495 & .8407 & .8671 & .8931 & $H_{3-10}$ & .0032 & .9201 & .9444 & .9516 \\
\hline
\end{tabular}

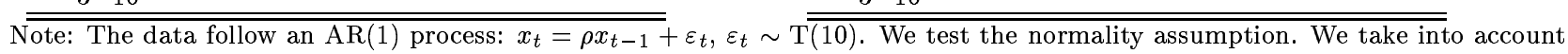
the serial correlation in the tests. We assume that we do not know the AR(1) dynamics. We use a HAC method. The results are based on 50000 replications. For each sample size, we provide the frequency of rejection at a $5 \%$ level. The notations $H_{i-j}$ are defined in Table 5. 
Table 7e: Power of the tests under serial correlation against $T(6)$ innovations.

\begin{tabular}{|c|c|c|c|c|c|c|c|c|c|}
\hline \multicolumn{5}{|c|}{ Panel A: $\rho=.1$} & \multicolumn{5}{|c|}{ Panel B: $\rho=.5$} \\
\hline $\mathrm{T}$ & 100 & 250 & 500 & 1000 & $\mathrm{~T}$ & 100 & 250 & 500 & 1000 \\
\hline$H_{3}$ & .2102 & .4324 & .5973 & .7244 & $H_{3}$ & .0957 & .2330 & .4013 & .6008 \\
\hline$H_{4}$ & .8263 & .9878 & .9999 & 1.0000 & $H_{4}$ & .7719 & .9512 & .9969 & 1.0000 \\
\hline$H_{5}$ & .6667 & .8287 & .9336 & .9791 & $H_{5}$ & .6891 & .7714 & .8544 & .9259 \\
\hline$H_{6}$ & .6985 & .9196 & .9901 & .9998 & $H_{6}$ & .7109 & .8853 & .9721 & .9982 \\
\hline$H_{7}$ & .6633 & .8888 & .9745 & .9956 & $H_{7}$ & .6728 & .8347 & .9323 & .9796 \\
\hline$H_{8}$ & .6002 & .8352 & .9541 & .9964 & $H_{8}$ & .6267 & .8050 & .9182 & .9818 \\
\hline$H_{9}$ & .5557 & .8062 & .9374 & .9904 & $H_{9}$ & .5713 & .7626 & .8865 & .9651 \\
\hline$H_{10}$ & .5285 & .7663 & .9137 & .9856 & $H_{10}$ & .5396 & .7297 & .8633 & .9541 \\
\hline$H_{3-4}$ & .1185 & .1095 & .1148 & .1315 & $H_{3-4}$ & .0826 & .0859 & .0990 & .1320 \\
\hline$H_{3-5}$ & .0627 & .2002 & .3715 & .5162 & $H_{3-5}$ & .0287 & .1016 & .2532 & .4411 \\
\hline$H_{3-6}$ & .4546 & .6419 & .8304 & .9327 & $H_{3-6}$ & .4587 & .5644 & .6791 & .8125 \\
\hline$H_{3-7}$ & .5990 & .8667 & .9802 & .9996 & $H_{3-7}$ & .6120 & .8296 & .9524 & .9965 \\
\hline$H_{3-8}$ & .5929 & .8324 & .9524 & .9896 & $H_{3-8}$ & .6077 & .7744 & .8918 & .9591 \\
\hline$H_{3-9}$ & .5539 & .7977 & .9379 & .9941 & $H_{3-9}$ & .5803 & .7686 & .8965 & .9752 \\
\hline$H_{3-10}$ & .5062 & .7457 & .9042 & .9828 & $H_{3-10}$ & .5312 & .7195 & .8520 & .9467 \\
\hline \multicolumn{5}{|c|}{ Panel C: $\rho=.7$} & \multicolumn{5}{|c|}{ Panel D: $\rho=.9$} \\
\hline $\mathrm{T}$ & 100 & 250 & 500 & 1000 & $\mathrm{~T}$ & 100 & 250 & 500 & 1000 \\
\hline$H_{3}$ & .0330 & .0964 & .1761 & .3087 & $H_{3}$ & .0014 & .0060 & .0144 & .0333 \\
\hline$H_{4}$ & .7186 & .8727 & .9586 & .9959 & $H_{4}$ & .0401 & .8695 & .9167 & .9400 \\
\hline$H_{5}$ & .7218 & .7883 & .8163 & .8519 & $H_{5}$ & .0359 & .8941 & .9260 & .9346 \\
\hline$H_{6}$ & .7137 & .8539 & .9311 & .9828 & $H_{6}$ & .0634 & .8955 & .9309 & .9473 \\
\hline$H_{7}$ & .6979 & .8222 & .8886 & .9449 & $H_{7}$ & .0862 & .8940 & .9267 & .9412 \\
\hline$H_{8}$ & .6617 & .8055 & .8831 & .9460 & $H_{8}$ & .0355 & .8823 & .9222 & .9390 \\
\hline$H_{9}$ & .6043 & .7732 & .8521 & .9217 & $H_{9}$ & .0126 & .8580 & .9145 & .9330 \\
\hline$H_{10}$ & .5406 & .7352 & .8282 & .9067 & $H_{10}$ & .0060 & .8217 & .9048 & .9265 \\
\hline$H_{3-4}$ & .0500 & .0651 & .0748 & .0966 & $H_{3-4}$ & .0376 & .0153 & .0204 & .0270 \\
\hline$H_{3-5}$ & .0125 & .0249 & .0796 & .2158 & $H_{3-5}$ & .0017 & .0018 & .0017 & .0037 \\
\hline$H_{3-6}$ & .4083 & .6198 & .6407 & .6738 & $H_{3-6}$ & .0077 & .4973 & .8678 & .8952 \\
\hline$H_{3-7}$ & .6140 & .8006 & .9019 & .9729 & $H_{3-7}$ & .0143 & .8469 & .9145 & .9363 \\
\hline$H_{3-8}$ & .6371 & .7770 & .8500 & .9121 & $H_{3-8}$ & .0285 & .8691 & .9163 & .9335 \\
\hline$H_{3-9}$ & .6142 & .7789 & .8629 & .9342 & $H_{3-9}$ & .0163 & .8597 & .9144 & .9333 \\
\hline$H_{3-10}$ & .5630 & .7451 & .8283 & .9008 & $H_{3-10}$ & .0078 & .8343 & .9077 & .9279 \\
\hline
\end{tabular}

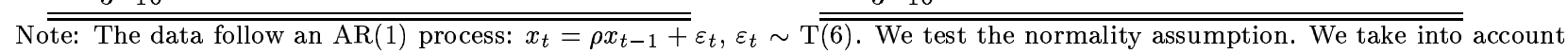
the serial correlation in the tests. We assume that we do not know the AR(1) dynamics. We use a HAC method. The results are based on 50000 replications. For each sample size, we provide the frequency of rejection at a $5 \%$ level. The notations $H_{i-j}$ are defined in Table 5. 
Table 7f: Power of the tests under serial correlation against $T(3)$ innovations.

\begin{tabular}{|c|c|c|c|c|c|c|c|c|c|}
\hline \multicolumn{5}{|c|}{ Panel A: $\rho=.1$} & \multicolumn{5}{|c|}{ Panel B: $\rho=.5$} \\
\hline $\mathrm{T}$ & 100 & 250 & 500 & 1000 & $\mathrm{~T}$ & 100 & 250 & 500 & 1000 \\
\hline$H_{3}$ & .2465 & .4043 & .5058 & .5951 & $H_{3}$ & .1607 & .3311 & .4694 & .5726 \\
\hline$H_{4}$ & .9494 & .9998 & 1.0000 & 1.0000 & $H_{4}$ & .8512 & .9972 & 1.0000 & 1.0000 \\
\hline$H_{5}$ & .7845 & .9683 & .9938 & .9977 & $H_{5}$ & .6610 & .9060 & .9808 & .9947 \\
\hline$H_{6}$ & .8228 & .9899 & .9999 & 1.0000 & $H_{6}$ & .7100 & .9637 & .9986 & 1.0000 \\
\hline$H_{7}$ & .8110 & .9859 & .9990 & .9999 & $H_{7}$ & .6877 & .9472 & .9946 & .9995 \\
\hline$H_{8}$ & .7411 & .9704 & .9991 & 1.0000 & $H_{8}$ & .6167 & .9153 & .9915 & .9999 \\
\hline$H_{9}$ & .7081 & .9591 & .9970 & 1.0000 & $H_{9}$ & .5648 & .8899 & .9844 & .9994 \\
\hline$H_{10}$ & .6710 & .9402 & .9959 & 1.0000 & $H_{10}$ & .5293 & .8645 & .9773 & .9992 \\
\hline$H_{3-4}$ & .0756 & .0309 & .0135 & .0056 & $H_{3-4}$ & .0880 & .0487 & .0296 & .0170 \\
\hline$H_{3-5}$ & .0893 & .1807 & .2523 & .3223 & $H_{3-5}$ & .0730 & .1557 & .2371 & .3142 \\
\hline$H_{3-6}$ & .5632 & .9090 & .9789 & .9900 & $H_{3-6}$ & .4129 & .7762 & .9425 & .9804 \\
\hline$H_{3-7}$ & .7403 & .9795 & .9997 & 1.0000 & $H_{3-7}$ & .6047 & .9356 & .9973 & 1.0000 \\
\hline$H_{3-8}$ & .7462 & .9732 & .9974 & .9993 & $H_{3-8}$ & .6119 & .9164 & .9888 & .9982 \\
\hline$H_{3-9}$ & .6941 & .9583 & .9985 & 1.0000 & $H_{3-9}$ & .5666 & .8919 & .9879 & .9998 \\
\hline$H_{3-10}$ & .6363 & .9331 & .9951 & .9999 & $H_{3-10}$ & .5081 & .8530 & .9760 & .9988 \\
\hline \multicolumn{5}{|c|}{ Panel C: $\rho=.7$} & \multicolumn{5}{|c|}{ Panel D: $\rho=.9$} \\
\hline $\mathrm{T}$ & 100 & 250 & 500 & 1000 & $\mathrm{n}$ & 100 & 250 & 500 & 1000 \\
\hline$H_{3}$ & .0704 & .1917 & .3516 & .5074 & $H_{3}$ & .0036 & .0183 & .0449 & .0914 \\
\hline$H_{4}$ & .6338 & .9452 & .9978 & 1.0000 & $H_{4}$ & .0465 & .6330 & .8661 & .9644 \\
\hline$H_{5}$ & .5083 & .7425 & .8995 & .9773 & $H_{5}$ & .0491 & .6044 & .7306 & .7803 \\
\hline$H_{6}$ & .5239 & .8596 & .9812 & .9997 & $H_{6}$ & .0609 & .6041 & .8310 & .9427 \\
\hline$H_{7}$ & .5138 & .8247 & .9596 & .9951 & $H_{7}$ & .0624 & .6004 & .7863 & .8870 \\
\hline$H_{8}$ & .4549 & .7854 & .9476 & .9961 & $H_{8}$ & .0378 & .5582 & .7873 & .9107 \\
\hline$H_{9}$ & .3856 & .7306 & .9185 & .9904 & $H_{9}$ & .0193 & .4771 & .7350 & .8723 \\
\hline$H_{10}$ & .3371 & .6859 & .8964 & .9870 & $H_{10}$ & .0135 & .3971 & .6777 & .8501 \\
\hline$H_{3-4}$ & .0848 & .0687 & .0527 & .0397 & $H_{3-4}$ & .0942 & .0358 & .0373 & .0361 \\
\hline$H_{3-5}$ & .0586 & .1169 & .1970 & .2834 & $H_{3-5}$ & .0163 & .0281 & .0348 & .0717 \\
\hline$H_{3-6}$ & .2423 & .5219 & .7737 & .9325 & $H_{3-6}$ & .0218 & .1973 & .4509 & .5501 \\
\hline$H_{3-7}$ & .4066 & .7886 & .9672 & .9993 & $H_{3-7}$ & .0391 & .4498 & .7428 & .9112 \\
\hline$H_{3-8}$ & .4394 & .7655 & .9356 & .9895 & $H_{3-8}$ & .0324 & .5073 & .7209 & .8356 \\
\hline$H_{3-9}$ & .3989 & .7463 & .9329 & .9943 & $H_{3-9}$ & .0246 & .4834 & .7425 & .8891 \\
\hline$H_{3-10}$ & .3473 & .6911 & .8968 & .9852 & $H_{3-10}$ & .0149 & .4220 & .6901 & .8410 \\
\hline
\end{tabular}

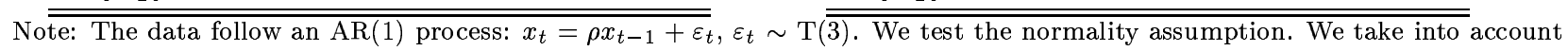
the serial correlation in the tests. We assume that we do not know the AR(1) dynamics. We use a HAC method. The results are based on 50000 replications. For each sample size, we provide the frequency of rejection at a $5 \%$ level. The notations $H_{i-j}$ are defined in Table 5. 
Table 7g: Power of the tests under serial correlation against $T(5)$ innovations.

\begin{tabular}{lllll}
\hline \hline \multicolumn{5}{c}{ Panel A: $\rho=.1$} \\
\hline & 100 & 250 & 500 & 1000 \\
$H_{3}$ & .2288 & .4567 & .6103 & .7263 \\
$H_{4}$ & .8575 & .9951 & 1.000 & 1.000 \\
$H_{5}$ & .6746 & .8674 & .9614 & .9885 \\
$H_{6}$ & .7142 & .9404 & .9955 & 1.000 \\
$H_{7}$ & .6845 & .9178 & .9881 & .9980 \\
$H_{8}$ & .6167 & .8710 & .9774 & .9989 \\
$H_{9}$ & .5725 & .8481 & .9639 & .9965 \\
$H_{10}$ & .5406 & .8099 & .9487 & .9948 \\
$H_{3-4}$ & .1128 & .0928 & .0834 & .0821 \\
$H_{3-5}$ & .0761 & .2228 & .3642 & .4884 \\
$H_{3-6}$ & .4488 & .7056 & .8921 & .9585 \\
$H_{3-7}$ & .6148 & .9004 & .9912 & .9999 \\
$H_{3-8}$ & .6118 & .8729 & .9751 & .9950 \\
$H_{3-9}$ & .5677 & .8385 & .9665 & .9984 \\
$H_{3-10}$ & .5148 & .7891 & .9422 & .9939 \\
\hline \hline
\end{tabular}

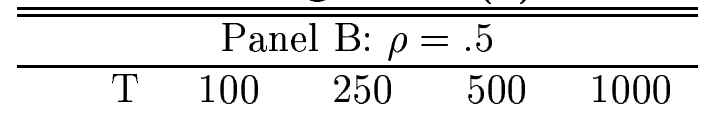

\begin{tabular}{ccccc}
\hline \hline \multicolumn{5}{c}{ Panel C: $\rho=.7$} \\
\hline T & 100 & 250 & 500 & 1000
\end{tabular}

\begin{tabular}{lllll}
$H_{3}$ & .1111 & .2677 & .4592 & .6404 \\
$H_{4}$ & .7775 & .9679 & .9992 & 1.000 \\
$H_{5}$ & .6500 & .7749 & .8874 & .9581 \\
$H_{6}$ & .6840 & .8994 & .9842 & .9996 \\
$H_{7}$ & .6458 & .8492 & .9536 & .9904 \\
$H_{8}$ & .5918 & .8157 & .9404 & .9926 \\
$H_{9}$ & .5328 & .7730 & .9147 & .9823 \\
$H_{10}$ & .5062 & .7384 & .8908 & .9766 \\
$H_{3-4}$ & .0875 & .0816 & .0880 & .1018 \\
$H_{3-5}$ & .0422 & .1328 & .2879 & .4450 \\
$H_{3-6}$ & .4121 & .5661 & .7380 & .8829 \\
$H_{3-7}$ & .5773 & .8417 & .9706 & .9992 \\
$H_{3-8}$ & .5732 & .7883 & .9224 & .9791 \\
$H_{3-9}$ & .5412 & .7785 & .9225 & .9892 \\
$H_{3-10}$ & .4901 & .7256 & .8813 & .9723 \\
\hline \hline
\end{tabular}

\begin{tabular}{llllllllll}
$H_{3}$ & .0395 & .1133 & .2158 & .3999 & $H_{3}$ & .0019 & .0076 & .0203 & .0428 \\
$H_{4}$ & .6805 & .8820 & .9733 & .9989 & $H_{4}$ & .0424 & .8341 & .9067 & .9366 \\
$H_{5}$ & .6541 & .7473 & .7962 & .8602 & $H_{5}$ & .0403 & .8623 & .9049 & .9119 \\
$H_{6}$ & .6489 & .8448 & .9426 & .9905 & $H_{6}$ & .0639 & .8643 & .9172 & .9427 \\
$H_{7}$ & .6310 & .7999 & .8927 & .9537 & $H_{7}$ & .0794 & .8625 & .9094 & .9312 \\
$H_{8}$ & .5874 & .7842 & .8888 & .9637 & $H_{8}$ & .0370 & .8454 & .9058 & .9292 \\
$H_{9}$ & .5230 & .7394 & .8525 & .9383 & $H_{9}$ & .0154 & .8057 & .8943 & .9204 \\
$H_{10}$ & .4588 & .6939 & .8228 & .9263 & $H_{10}$ & .0080 & .7507 & .8802 & .9114 \\
$H_{3-4}$ & .0581 & .0684 & .0737 & .0893 & $H_{3-4}$ & .0497 & .0179 & .0240 & .0311 \\
$H_{3-5}$ & .0211 & .0448 & .1260 & .2903 & $H_{3-5}$ & .0029 & .0030 & .0036 & .0066 \\
$H_{3-6}$ & .3406 & .5429 & .5934 & .6874 & $H_{3-6}$ & .0107 & .4078 & .8109 & .8456 \\
$H_{3-7}$ & .5348 & .7793 & .9121 & .9850 & $H_{3-7}$ & .0186 & .7872 & .8949 & .9268 \\
$H_{3-8}$ & .5607 & .7443 & .8446 & .9214 & $H_{3-8}$ & .0306 & .8223 & .8943 & .9162 \\
$H_{3-9}$ & .5331 & .7490 & .8669 & .9542 & $H_{3-9}$ & .0191 & .8084 & .8951 & .9219 \\
$H_{3-10}$ & .4786 & .7041 & .8223 & .9170 & $H_{3-10}$ & .0102 & .7689 & .8841 & .9123 \\
\hline \hline
\end{tabular}

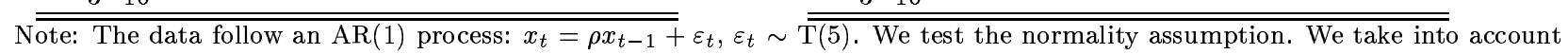
the serial correlation in the tests. We assume that we do not know the AR(1) dynamics. We use a HAC method. The results are based on 50000 replications. For each sample size, we provide the frequency of rejection at a $5 \%$ level. The notations $H_{i-j}$ are defined in Table 5. 
Table 8: Testing $\mathcal{N}(0,1)$ of fitted residuals for a $\operatorname{GARCH}(\mathbf{1}, \mathbf{1})$ model.

\begin{tabular}{|l|r|r|r|r|}
\hline & UK-US $\$$ & FF-US $\$$ & SF-US $\$$ & Yen-US $\$$ \\
\hline$H_{3}$ & $1.86(.173)$ & $1.24(.264)$ & $51.0(.000)$ & $17.5(.000)$ \\
$H_{4}$ & $42.6(.000)$ & $38.7(.000)$ & $189(.000)$ & $577(.000)$ \\
$H_{5}$ & $9.53(.002)$ & $15.5(.000)$ & $590(.000)$ & $3713(.000)$ \\
$H_{6}$ & $46.8(.000)$ & $126(.000)$ & $2562(.000)$ & $28553(.000)$ \\
$H_{7}$ & $26.9(.000)$ & $8.7(.003)$ & $10956(.000)$ & $181020(.000)$ \\
$H_{8}$ & $45.9(.000)$ & $8.5(.000)$ & $35135(.000)$ & $945122(.000)$ \\
$H_{9}$ & $17.2(.000)$ & $2.35(.125)$ & $88029.039(.000)$ & $4186878.386(.000)$ \\
$H_{10}$ & $9.13(.003)$ & $42.9(.000)$ & $177511(.000)$ & $15683206(.000)$ \\
$H_{3-4}$ & $44.4(.000)$ & $39.9(.000)$ & $240(.000)$ & $594(.000)$ \\
$H_{3-5}$ & $54.1(.000)$ & $55.4(.000)$ & $830(.000)$ & $4308(.000)$ \\
$H_{3-6}$ & $100(.000)$ & $182(.000)$ & $3393(.000)$ & $32861(.000)$ \\
$H_{3-7}$ & $127(.000)$ & $191(.000)$ & $14349(.000)$ & $213882(.000)$ \\
$H_{3-8}$ & $173(.000)$ & $271(.000)$ & $49485(.000)$ & $1159004(.000)$ \\
$H_{3-9}$ & $191(.000)$ & $273(.000)$ & $137514(.000)$ & $5345882(.000)$ \\
$H_{3-10}$ & $200(.000)$ & $316(.000)$ & $315026(.000)$ & $21029088(.000)$ \\
$\mathrm{KS}$ & $1.01(.010)$ & $.754(1.00)$ & $1.29(.010)$ & $1.07(.010)$ \\
$\mathrm{JB}$ & $44.6(.000)$ & $4.1(.000)$ & $240(.000)$ & $597(.000)$ \\
\hline \hline
\end{tabular}

Note: We test the $\mathcal{N}(0,1)$ assumption of the standardized residuals. The volatility model is a GARCH $(1,1)$ and is estimated by the Guassian QML method. We report the test statistics and their corresponding p-values in parenthese. The data are daily exchange rate returns used by Harvey, Ruiz and Shephard (1994) and Kim, Shephard and Chib (1998). $H_{i-j}$ is the joint test based on $H_{k}, i \leq k \leq j$. KS and JB are the Kolmogorov-Smirnov and Jarque-Bera tests. 
Table 9a: Testing $\mathcal{N}(0,1)$ of stantardized residuals by realized volatility.

Panel A: i.i.d. assumption.

\begin{tabular}{|l|rr|rr|rr|}
\hline & DM-US\$-5 & DM-US $\$-30$ & Yen-US $\$-5$ & Yen-US $\$-30$ & Yen-DM-5 & Yen-DM-30 \\
\hline$H_{3}$ & $.259(.6106)$ & $.450(.5023)$ & $.019(.8910)$ & $.759(.3837)$ & $.172(.6780)$ & $.076(.7822)$ \\
$H_{4}$ & $6.57(.0104)$ & $6.69(.0097)$ & $3.83(.0503)$ & $8.91(.0028)$ & $1.99(.1577)$ & $11.0(.0009)$ \\
$H_{5}$ & $1.24(.2649)$ & $.507(.4764)$ & $.523(.4696)$ & $.435(.5095)$ & $.074(.7859)$ & $.004(.9504)$ \\
$H_{6}$ & $1.24(.2661)$ & $2.38(.1228)$ & $.296(.5865)$ & $2.26(.1325)$ & $.019(.8916)$ & $6.18(.0129)$ \\
$H_{7}$ & $1.29(.2566)$ & $.274(.6006)$ & $.590(.4424)$ & $.157(.6923)$ & $.039(.8427)$ & $.035(.8510)$ \\
$H_{8}$ & $.710(.3994)$ & $.041(.8397)$ & $.153(.6961)$ & $.003(.9593)$ & $.057(.8106)$ & $1.31(.2526)$ \\
$H_{9}$ & $.533(.4653)$ & $.074(.7851)$ & $.207(.6491)$ & $.111(.7390)$ & $.038(.8446)$ & $.007(.9337)$ \\
$H_{10}$ & $.501(.4791)$ & $.125(.7239)$ & $.223(.6370)$ & $.354(.5517)$ & $.143(.7055)$ & $.035(.8511)$ \\
$H_{3-4}$ & $6.82(.0330)$ & $7.14(.0281)$ & $3.85(.1458)$ & $9.67(.0080)$ & $2.17(.3382)$ & $11.1(.0040)$ \\
$H_{3-5}$ & $8.07(.0447)$ & $7.65(.0539)$ & $4.37(.2238)$ & $1.1(.0177)$ & $2.24(.5237)$ & $11.1(.0114)$ \\
$H_{3-6}$ & $9.30(.0540)$ & $1.0(.0399)$ & $4.67(.3229)$ & $12.4(.0148)$ & $2.26(.6880)$ & $17.2(.0017)$ \\
$H_{3-7}$ & $1.6(.0602)$ & $1.3(.0671)$ & $5.26(.3850)$ & $12.5(.0283)$ & $2.30(.8063)$ & $17.3(.0040)$ \\
$H_{3-8}$ & $11.3(.0795)$ & $1.3(.1109)$ & $5.41(.4921)$ & $12.5(.0512)$ & $2.36(.8841)$ & $18.6(.0049)$ \\
$H_{3-9}$ & $11.8(.1062)$ & $1.4(.1660)$ & $5.62(.5848)$ & $12.6(.0815)$ & $2.40(.9347)$ & $18.6(.0096)$ \\
$H_{3-10}$ & $12.3(.1369)$ & $1.5(.2289)$ & $5.84(.6649)$ & $13.0(.1122)$ & $2.54(.9599)$ & $18.6(.0170)$ \\
$\mathrm{KS}$ & $2.52(.0100)$ & $.598(1.000)$ & $2.43(.0100)$ & $.519(1.000)$ & $1.99(.0100)$ & $.788(1.000)$ \\
$\mathrm{JB}$ & $.998(.6073)$ & $11.9(.0026)$ & $.819(.6640)$ & $13.5(.0011)$ & $2.14(.3428)$ & $16.4(.0003)$ \\
\hline \hline
\end{tabular}

Panel B: serial correlation.

\begin{tabular}{|l|rr|rr|rr|}
\hline & DM-US\$-5 & DM-US\$-30 & Yen-US\$-5 & Yen-US\$-30 & Yen-DM-5 & Yen-DM-30 \\
\hline$H_{3}$ & $.623(.4300)$ & $.889(.3459)$ & $.046(.8303)$ & $1.43(.2319)$ & $.425(.5146)$ & $.138(.7105)$ \\
$H_{4}$ & $17.8(.0000)$ & $17.7(.0000)$ & $8.99(.0027)$ & $19.4(.0000)$ & $5.65(.0175)$ & $21.2(.0000)$ \\
$H_{5}$ & $3.87(.0492)$ & $1.30(.2533)$ & $1.66(.1975)$ & $1.09(.2973)$ & $.224(.6362)$ & $.009(.9240)$ \\
$H_{6}$ & $4.13(.0420)$ & $6.83(.0090)$ & $.963(.3265)$ & $6.22(.0127)$ & $.060(.8069)$ & $15.2(.0001)$ \\
$H_{7}$ & $4.77(.0290)$ & $.913(.3392)$ & $2.04(.1531)$ & $.457(.4991)$ & $.142(.7059)$ & $.095(.7583)$ \\
$H_{8}$ & $2.87(.0904)$ & $.140(.7087)$ & $.590(.4426)$ & $.008(.9272)$ & $.209(.6472)$ & $3.52(.0606)$ \\
$H_{9}$ & $2.24(.1346)$ & $.259(.6105)$ & $.817(.3660)$ & $.364(.5463)$ & $.157(.6924)$ & $.024(.8777)$ \\
$H_{10}$ & $2.24(.1344)$ & $.495(.4817)$ & $.947(.3304)$ & $1.25(.2640)$ & $.595(.4405)$ & $.114(.7351)$ \\
$H_{3-4}$ & $19.9(.0000)$ & $18.6(.0001)$ & $1.1(.0064)$ & $22.1(.0000)$ & $6.23(.0445)$ & $22.5(.0000)$ \\
$H_{3-5}$ & $23.2(.0000)$ & $19.1(.0003)$ & $11.9(.0079)$ & $22.2(.0001)$ & $6.26(.0996)$ & $24.5(.0000)$ \\
$H_{3-6}$ & $32.2(.0000)$ & $2.0(.0005)$ & $19.7(.0006)$ & $24.2(.0001)$ & $15.2(.0043)$ & $24.8(.0001)$ \\
$H_{3-7}$ & $32.8(.0000)$ & $2.0(.0013)$ & $19.7(.0014)$ & $25.3(.0001)$ & $15.7(.0076)$ & $25.2(.0001)$ \\
$H_{3-8}$ & $65.1(.0000)$ & $23.1(.0007)$ & $46.3(.0000)$ & $25.5(.0003)$ & $47.5(.0000)$ & $25.3(.0003)$ \\
$H_{3-9}$ & $76.0(.0000)$ & $23.6(.0013)$ & $46.5(.0000)$ & $27.7(.0002)$ & $48.1(.0000)$ & $25.4(.0006)$ \\
$H_{3-10}$ & $178 .(.0000)$ & $73.9(.0000)$ & $66.4(.0000)$ & $33.3(.0001)$ & $146 .(.0000)$ & $25.4(.0013)$ \\
\hline
\end{tabular}

Note: We test the $\mathcal{N}(0,1)$ assumption of the standardized residuals. The volatility is the daily realized volatility computed with

five-minute and thirty-minute returns. We report the test statistics and their corresponding p-values in parenthese. We use the same dat as ABDL (2002). In Panel A, we assume that the residuals are i.i.d., while we allow them to be serially correlated in Panel B. In this case, we use a HAC method of Andrews (1991) to estimate the weighting matrix. $H_{i-j}$ is the joint test based on $H_{k}, i \leq k \leq j$. 
Table 9b: Testing normality of stantardized residuals by realized volatility.

Panel A: i.i.d. assumption.

\begin{tabular}{|l|rr|rr|rr|}
\hline & DM-US\$-5 & DM-US\$-30 & Yen-US $\$-5$ & Yen-US $\$-30$ & Yen-DM-5 & Yen-DM-30 \\
\hline$H_{3}$ & $.789(.3744)$ & $.550(.4584)$ & $.142(.7068)$ & $.930(.3348)$ & $.154(.6947)$ & $.128(.7203)$ \\
$H_{4}$ & $.011(.9151)$ & $11.7(.0006)$ & $.172(.6785)$ & $11.5(.0007)$ & $1.99(.1581)$ & $14.8(.0001)$ \\
$H_{5}$ & $3.31(.0690)$ & $.600(.4387)$ & $1.76(.1847)$ & $.334(.5634)$ & $.040(.8422)$ & $.061(.8051)$ \\
$H_{6}$ & $1.27(.2601)$ & $1.29(.2559)$ & $.173(.6778)$ & $1.66(.1981)$ & $.177(.6738)$ & $5.42(.0199)$ \\
$H_{7}$ & $.684(.4081)$ & $.232(.6298)$ & $.312(.5767)$ & $.108(.7423)$ & $.023(.8784)$ & $.093(.7600)$ \\
$H_{8}$ & $.600(.4385)$ & $.094(.7586)$ & $.317(.5731)$ & $.049(.8242)$ & $.608(.4355)$ & $.695(.4044)$ \\
$H_{9}$ & $.141(.7073)$ & $.027(.8705)$ & $.091(.7629)$ & $.111(.7392)$ & $.061(.8049)$ & $.008(.9290)$ \\
$H_{10}$ & $.165(.6842)$ & $.308(.5787)$ & $.536(.4641)$ & $.540(.4625)$ & $.138(.7107)$ & $.006(.9372)$ \\
$H_{3-4}$ & $.800(.6702)$ & $12.2(.0022)$ & $.313(.8550)$ & $12.5(.0020)$ & $2.15(.3419)$ & $15.0(.0006)$ \\
$H_{3-5}$ & $4.11(.2500)$ & $12.8(.0050)$ & $2.07(.5574)$ & $12.8(.0051)$ & $2.19(.5346)$ & $15.0(.0018)$ \\
$H_{3-6}$ & $5.38(.2508)$ & $14.1(.0069)$ & $2.25(.6907)$ & $14.5(.0059)$ & $2.36(.6692)$ & $2.4(.0004)$ \\
$H_{3-7}$ & $6.06(.3004)$ & $14.4(.0135)$ & $2.56(.7678)$ & $14.6(.0123)$ & $2.39(.7934)$ & $2.5(.0010)$ \\
$H_{3-8}$ & $6.66(.3534)$ & $14.5(.0250)$ & $2.87(.8244)$ & $14.6(.0234)$ & $2.99(.8095)$ & $21.2(.0017)$ \\
$H_{3-9}$ & $6.80(.4498)$ & $14.5(.0433)$ & $2.97(.8881)$ & $14.7(.0396)$ & $3.06(.8798)$ & $21.2(.0034)$ \\
$H_{3-10}$ & $6.97(.5402)$ & $14.8(.0634)$ & $3.50(.8990)$ & $15.3(.0540)$ & $3.19(.9216)$ & $21.3(.0065)$ \\
$\mathrm{KS}$ & $.797(1.000)$ & $.682(1.000)$ & $.646(1.000)$ & $.713(1.000)$ & $.485(1.000)$ & $.894(.0100)$ \\
$\mathrm{JB}$ & $.801(.6699)$ & $12.3(.0022)$ & $.314(.8548)$ & $12.5(.0019)$ & $2.15(.3413)$ & $15.0(.0006)$ \\
\hline \hline
\end{tabular}

Panel B: serial correlation.

\begin{tabular}{|l|rr|rr|rr|}
\hline & DM-US\$-5 & DM-US\$-30 & Yen-US $\$-5$ & Yen-US $\$-30$ & Yen-DM-5 & Yen-DM-30 \\
\hline$H_{3}$ & $.863(.3528)$ & $.874(.3498)$ & $.160(.6896)$ & $1.53(.2158)$ & $.204(.6513)$ & $.205(.6505)$ \\
$H_{4}$ & $.014(.9043)$ & $27.0(.0000)$ & $.21(.6471)$ & $23.1(.0000)$ & $3.35(.0671)$ & $24.8(.0000)$ \\
$H_{5}$ & $5.93(.0149)$ & $1.44(.2294)$ & $2.65(.1038)$ & $.778(.3779)$ & $.081(.7754)$ & $.127(.7219)$ \\
$H_{6}$ & $2.70(.1000)$ & $3.15(.0760)$ & $.341(.5592)$ & $4.20(.0405)$ & $.360(.5487)$ & $12.3(.0005)$ \\
$H_{7}$ & $1.46(.2272)$ & $.668(.4136)$ & $.653(.4190)$ & $.284(.5939)$ & $.053(.8174)$ & $.233(.6296)$ \\
$H_{8}$ & $1.29(.2557)$ & $.291(.5893)$ & $.645(.4219)$ & $.147(.7016)$ & $1.56(.2112)$ & $1.70(.1918)$ \\
$H_{9}$ & $.335(.5626)$ & $.079(.7789)$ & $.198(.6563)$ & $.346(.5562)$ & $.172(.6785)$ & $.026(.8728)$ \\
$H_{10}$ & $.439(.5077)$ & $1.07(.2998)$ & $1.35(.2451)$ & $1.76(.1849)$ & $.396(.5292)$ & $.019(.8907)$ \\
$H_{3-4}$ & $.968(.6163)$ & $27.9(.0000)$ & $.309(.8568)$ & $26.2(.0000)$ & $3.65(.1609)$ & $26.8(.0000)$ \\
$H_{3-5}$ & $6.31(.0973)$ & $28.6(.0000)$ & $3.60(.3077)$ & $26.8(.0000)$ & $3.66(.3009)$ & $3.0(.0000)$ \\
$H_{3-6}$ & $14.6(.0057)$ & $39.1(.0000)$ & $5.98(.2005)$ & $32.6(.0000)$ & $12.2(.0157)$ & $3.1(.0000)$ \\
$H_{3-7}$ & $18.7(.0021)$ & $39.2(.0000)$ & $8.04(.1538)$ & $35.8(.0000)$ & $12.2(.0315)$ & $32.1(.0000)$ \\
$H_{3-8}$ & $21.0(.0018)$ & $4.4(.0000)$ & $9.98(.1254)$ & $36.6(.0000)$ & $19.0(.0041)$ & $35.4(.0000)$ \\
$H_{3-9}$ & $21.4(.0032)$ & $4.7(.0000)$ & $11.8(.1057)$ & $37.2(.0000)$ & $19.3(.0074)$ & $36.0(.0000)$ \\
$H_{3-10}$ & $22.7(.0038)$ & $47.3(.0000)$ & $18.4(.0185)$ & $38.0(.0000)$ & $19.8(.0112)$ & $36.8(.0000)$ \\
\hline
\end{tabular}

Note: We test the normality assumption of the standardized residuals. The volatility is the daily realized volatility computed

with five-minute and thirty-minute returns. We report the test statistics and their corresponding p-values in parenthese. We use the same dat as ABDL (2002). In Panel A, we assume that the residuals are i.i.d., while we allow them to be serially correlated in Panel B. In this case, we use a HAC method of Andrews (1991) to estimate the weighting matrix. $H_{i-j}$ is the joint test based on $H_{k}, i \leq k \leq j$. 
Table 10: Testing log-normality of realized volatility.

\begin{tabular}{|l|rr|rr|rr|}
\hline & DM-US\$-5 & DM-US\$-30 & Yen-US\$-5 & Yen-US\$-30 & Yen-DM-5 & Yen-DM-30 \\
$H_{3}$ & $1.6(.0011)$ & $8.31(.0039)$ & $1.2(.0014)$ & $6.99(.0082)$ & $8.67(.0032)$ & $3.58(.0585)$ \\
$H_{4}$ & $7.46(.0063)$ & $5.91(.0151)$ & $3.61(.0572)$ & $3.41(.0646)$ & $5.17(.0230)$ & $3.37(.0664)$ \\
$H_{5}$ & $.149(.6998)$ & $2.92(.0872)$ & $1.14(.2858)$ & $2.19(.1393)$ & $.749(.3869)$ & $.002(.9685)$ \\
$H_{6}$ & $.000(.9966)$ & $.035(.8517)$ & $.732(.3923)$ & $.997(.3181)$ & $1.77(.1831)$ & $.997(.3181)$ \\
$H_{7}$ & $8.94(.0028)$ & $.827(.3633)$ & $1.01(.3163)$ & $1.65(.1984)$ & $.742(.3891)$ & $2.66(.1030)$ \\
$H_{8}$ & $.708(.4000)$ & $.476(.4903)$ & $.919(.3377)$ & $1.29(.2558)$ & $1.02(.3116)$ & $1.66(.1974)$ \\
$H_{9}$ & $1.46(.2265)$ & $.563(.4532)$ & $.007(.9323)$ & $4.00(.0455)$ & $8.14(.0043)$ & $.868(.3514)$ \\
$H_{10}$ & $.562(.4534)$ & $1.05(.3046)$ & $2.19(.1390)$ & $4.02(.0448)$ & $1.06(.3039)$ & $.417(.5182)$ \\
$H_{3-4}$ & $16.9(.0002)$ & $1.5(.0052)$ & $16.8(.0002)$ & $9.63(.0081)$ & $11.0(.0040)$ & $7.05(.0295)$ \\
$H_{3-5}$ & $17.6(.0005)$ & $1.5(.0145)$ & $17.4(.0006)$ & $9.79(.0204)$ & $17.3(.0006)$ & $7.74(.0518)$ \\
$H_{3-6}$ & $17.7(.0014)$ & $15.2(.0044)$ & $19.2(.0007)$ & $16.0(.0030)$ & $17.3(.0017)$ & $7.83(.0981)$ \\
$H_{3-7}$ & $24.0(.0002)$ & $15.5(.0084)$ & $26.2(.0001)$ & $24.5(.0002)$ & $18.6(.0023)$ & $1.1(.0715)$ \\
$H_{3-8}$ & $25.6(.0003)$ & $15.5(.0165)$ & $28.2(.0001)$ & $25.9(.0002)$ & $18.9(.0043)$ & $1.2(.1157)$ \\
$H_{3-9}$ & $25.9(.0005)$ & $17.5(.0147)$ & $28.3(.0002)$ & $26.4(.0004)$ & $25.6(.0006)$ & $12.1(.0968)$ \\
$H_{3-10}$ & $27.0(.0007)$ & $19.9(.0108)$ & $28.5(.0004)$ & $27.9(.0005)$ & $26.2(.0010)$ & $12.5(.1323)$ \\
\hline \hline
\end{tabular}

Note: We test the normality assumption of the log of realized volatility. The realized volatility is computed with five-minute

and thirty-minute returns. We report the test statistics and their corresponding p-values in parenthese. We use the same data as ABDL (2002). We use a HAC method of Andrews (1991) to estimate the weighting matrix. $H_{i-j}$ is the joint test based on $H_{k}$, $i \leq k \leq j$. 


\section{Appendix}

Proof of Proposition 2.1. Let assume that a random variable $x$ is such that $E\left[H_{i}(x)\right]=0$. We will prove that this variable follows the Stein equation (2.1) to prove the proposition.

Consider a polynomial $f$ of degree $n$; it can be written as a linear combination of the first $\mathrm{n}$ Hermite polynomials, i.e., it exist $\left(\alpha_{0}, \ldots, \alpha_{n}\right)$ such as: $f(x)=\sum_{i=0}^{n} \alpha_{i} H_{i}(x)$. Thus, using $(2.3)$ and (2.7), one gets:

$$
\begin{aligned}
f^{\prime}(x)-x f(x) & =\sum_{i=0}^{n} \alpha_{i} H_{i}^{\prime}(x)-\sum_{i=0}^{n} \alpha_{i}\left(x H_{i}(x)\right) \\
& =\sum_{i=1}^{n} \alpha_{i} \sqrt{i} H_{i-1}(x)-\alpha_{0} x-\sum_{i=1}^{n} \alpha_{i}\left(\sqrt{i} H_{i-1}(x)+\sqrt{i+1} H_{i+1}(x)\right) \\
& =-\alpha_{0} H_{1}-\sum_{i=1}^{n} \alpha_{i} \sqrt{i+1} H_{i+1}(x)=-\sum_{i=0}^{n} \alpha_{i} \sqrt{i+1} H_{i+1}(x),
\end{aligned}
$$

which implies that $E\left[f^{\prime}(x)-x f(x)\right]=0$. Consider now a continuously differentiable function $f$ whose derivative function is continuous $\left(f\right.$ is $\left.\mathcal{C}^{1}\right)$, such that $E\left|f^{\prime}(x)\right|<\infty$ and let $\varepsilon$ be a positive real number. $\forall(a, b) \in \mathbb{R}^{2}$, we have:

$$
\int_{-\infty}^{+\infty}\left(f^{\prime}(x)-x f(x)\right) \phi(x) d x=f(a) \phi(a)-f(b) \phi(b)+\int_{a}^{b}\left(f^{\prime}(x)-x f(x)\right) \phi(x) d x
$$

where $\phi(x)$ is the density function of a $\mathcal{N}(0,1)$ random variable. Given that $f^{\prime}$ is continuous function on the compact set $[a, b]$, the Stone-Weierstrass Theorem implies that:

$$
\exists N \in \mathbb{N} \forall n \geq N, \exists P_{n} \in \mathbb{R}^{n}[X],\left\|f^{\prime}-P_{n}\right\|_{\infty}<\varepsilon
$$

where $\left\|f^{\prime}-P_{n}\right\|_{\infty}=\sup _{x \in[a, b]}\left|f^{\prime}(x)-P_{n}(x)\right|$. and $\mathbb{R}^{n}[X]$ is the set of polynomials with degree $m \leq n$. Define the polynomial $Q_{n}(x)$ by $Q_{n}(x)=\int_{a}^{x} P_{n}(t) d t+f(a)$. Observe that $Q_{n}$ is a polynomial of degree $n+1, Q_{n}(a)=f(a)$ and $Q_{n}(b) \stackrel{n \rightarrow+\infty}{\longrightarrow} f(b)$. Hence, given that $E\left(P_{n}(x)-x Q_{n}(x)\right)=0$, one gets by integration by part:

$$
0=Q_{n}(a) \phi(a)-Q_{n}(b) \phi(b)+\int_{a}^{b}\left(P_{n}(x)-x Q_{n}(x)\right) \phi(x) d x .
$$

Hence, we have:

$$
\begin{aligned}
& E\left(f^{\prime}(x)-x f(x)\right)=E\left(f^{\prime}(x)-x f(x)\right)-E\left(P_{n}(x)-x Q_{n}(x)\right) \\
& =\left(Q_{n}(b)-f(b)\right) \phi(b)+\int_{a}^{b} \eta(x) \phi(x) d x-\int_{a}^{b} x \int_{a}^{x} \eta(t) d t \phi(x) d x,
\end{aligned}
$$

where $\eta(x)=f^{\prime}(x)-P_{n}(x)$. The first term of (A.2) tends to 0 when $n \rightarrow+\infty$. The second term of (A.2) is bounded by $\varepsilon \int_{-\infty}^{+\infty} \phi(x) d x$ while the third one is bounded by $\varepsilon \int_{-\infty}^{+\infty} x^{2} \phi(x) d x$. Hence, we get that $E\left(f^{\prime}(x)-x f(x)\right)=0$, which achieves the proof of the proposition. 
Proof of Proposition 4.1. Consider the first example and observe that

$$
g\left(z_{t}, \theta\right)=\tilde{g}\left(u_{t}(\theta)\right)=\tilde{g}\left(\frac{y_{t}-m\left(x_{t}, \theta\right)}{\sigma\left(x_{t}, \theta\right)}\right) .
$$

Then, we have:

$$
\begin{aligned}
& \frac{\partial g}{\partial \theta^{\top}}\left(z_{t}, \theta\right)=\tilde{g}^{\prime}\left(\frac{y_{t}-m\left(x_{t}, \theta\right)}{\sigma\left(x_{t}, \theta\right)}\right) \frac{\partial m}{\partial \theta^{\top}}\left(x_{t}, \theta\right)-\tilde{g}^{\prime}\left(\frac{y_{t}-m\left(x_{t}, \theta\right)}{\sigma\left(x_{t}, \theta\right)}\right) \frac{\left(y_{t}-m\left(x_{t}, \theta\right)\right)}{\sigma^{2}\left(x_{t}, \theta\right)} \frac{\partial \sigma}{\partial \theta^{\top}}\left(x_{t}, \theta\right) \\
& \quad=\tilde{g}^{\prime}\left(u_{t}(\theta)\right) \frac{\partial m}{\partial \theta^{\top}}\left(x_{t}, \theta\right)-\tilde{g}^{\prime}\left(u_{t}(\theta)\right) u_{t}(\theta) \frac{1}{\sigma\left(x_{t}, \theta\right)} \frac{\partial \sigma}{\partial \theta^{\top}}\left(x_{t}, \theta\right) .
\end{aligned}
$$

Hence, we have:

$E\left[\frac{\partial g}{\partial \theta^{\top}}\left(z_{t}, \theta^{0}\right)\right]=E\left[\tilde{g}^{\prime}\left(u_{t}\left(\theta^{0}\right)\right)\right] E\left[\frac{\partial m}{\partial \theta^{\top}}\left(x_{t}, \theta^{0}\right)\right]-E\left[\tilde{g}^{\prime}\left(u_{t}\left(\theta^{0}\right)\right) u_{t}\left(\theta^{0}\right)\right] E\left[\frac{1}{\sigma\left(x_{t}, \theta\right)} \frac{\partial \sigma}{\partial \theta^{\top}}\left(x_{t}, \theta^{0}\right)\right]$ since $x_{t}$ is an exogenous variable. Hence, under (4.15), we have $E\left[\frac{\partial g}{\partial \theta}\left(z_{t}, \theta^{0}\right)\right]=0$, i.e., (4.7) holds. This achieves the proof for the first example. The same proof holds for the second example since $u_{t}$ is independent of $y_{\tau}, \tau \leq t-1$.

Consider now the third example. We still have $\theta=(m, \sigma)$. Hence:

$\frac{\partial g}{\partial \theta^{\top}}\left(z_{t}, \theta\right)=\tilde{g}^{\prime}\left(u_{t}(\theta)\right)\left[\begin{array}{l}1 \\ 0\end{array}\right]-\tilde{g}^{\prime}\left(u_{t}(\theta)\right) u_{t}(\theta) \frac{1}{\sigma}\left[\begin{array}{l}0 \\ 1\end{array}\right]$, and $E\left[\frac{\partial g}{\partial \theta}\left(z_{t}, \theta^{0}\right)\right]=0$ under (4.15). This achieves the proof for the third example.

Proof of Proposition 4.2. Since $\tilde{g}(x)=f^{\prime}-x f(x)$, we have $\tilde{g}^{\prime}(x)=f^{\prime \prime}(x)-x f^{\prime}(x)-f(x)$ and $x \tilde{g}^{\prime}(x)=\left(x f^{\prime}(x)\right)^{\prime}-x\left(x f^{\prime}(x)\right)+f^{\prime}(x)-x f(x)-2 f^{\prime}(x)$. Applying the Stein equation $(2.1)$ to $f^{\prime}(x)$ and $x f^{\prime}(x)$ proves the proposition.

Proof of Proposition 4.3. The orthogonality property of the Hermite polynomials, i.e. (2.5), and (2.7) prove the proposition.

Proof of Proposition 5.1. Given that $Y \sim \mathcal{T}(\nu), E Y^{p}$ is well defined when $p<\nu$. In this case, we have $E Y^{p}=\nu^{p / 2} \Gamma\left(\frac{p+1}{2}\right) \Gamma\left(\frac{\nu-p}{2}\right) /\left(\Gamma\left(\frac{1}{2}\right) \Gamma\left(\frac{\nu}{2}\right)\right)$ if $p$ is even and $E Y^{p}=0$ otherwise.

Thus, for $\nu>8$, we have $E\left[H_{3}(X)\right]=\operatorname{Cov}\left(H_{3}(X), H_{4}(X)\right)=0, E\left[H_{4}(X)\right]=\sqrt{\frac{3}{2}} \frac{1}{\nu-4}$.

In addition, we have: $\operatorname{Var}\left(H_{3}(X)\right)=E H_{3}^{2}(X)=6^{-1}\left(E X^{6}-6 E X^{4}+9 E X^{2}\right)=A(\nu)$. The same computations lead to show that $\operatorname{Var}\left(H_{4}(X)\right)=B(\nu)$. This achieves the proof of (5.1). The results (5.3), (5.4) and (5.5) are implied by (5.1) 